\title{
Two NLR immune receptors acquired high-affinity binding to a fungal effector through convergent evolution of their integrated domain
}

\begin{abstract}
Aleksandra Białas ${ }^{1}$, Thorsten Langner ${ }^{1}$, Adeline Harant ${ }^{1}$, Mauricio P Contreras ${ }^{1}$, Clare EM Stevenson ${ }^{2}$, David M Lawson ${ }^{2}$, Jan Sklenar ${ }^{1}$, Ronny Kellner ${ }^{1}$, Matthew J Moscou1, Ryohei Terauchi ${ }^{3,4}$, Mark J Banfield ${ }^{2}$, Sophien Kamoun ${ }^{1 *}$
\end{abstract}

${ }^{1}$ The Sainsbury Laboratory, University of East Anglia, Norwich Research Park, Norwich, United Kingdom; ${ }^{2}$ Department of Biological Chemistry, John Innes Centre, Norwich Research Park, Norwich, United Kingdom; ${ }^{3}$ Division of Genomics and Breeding, Iwate Biotechnology Research Centre, Iwate, Japan; ${ }^{4}$ Laboratory of Crop Evolution, Graduate School of Agriculture, Kyoto University, Kyoto, Japan

Abstract A subset of plant NLR immune receptors carry unconventional integrated domains in addition to their canonical domain architecture. One example is rice Pik-1 that comprises an integrated heavy metal-associated (HMA) domain. Here, we reconstructed the evolutionary history of Pik-1 and its NLR partner, Pik-2, and tested hypotheses about adaptive evolution of the HMA domain. Phylogenetic analyses revealed that the HMA domain integrated into Pik-1 before Oryzinae speciation over 15 million years ago and has been under diversifying selection. Ancestral sequence reconstruction coupled with functional studies showed that two Pik-1 allelic variants independently evolved from a weakly binding ancestral state to high-affinity binding of the blast fungus effector AVR-PikD. We conclude that for most of its evolutionary history the Pik-1 HMA domain did not sense AVR-PikD, and that different Pik-1 receptors have recently evolved through distinct biochemical paths to produce similar phenotypic outcomes. These findings highlight the dynamic nature of the evolutionary mechanisms underpinning NLR adaptation to plant pathogens.

\section{Introduction}

Nucleotide-binding domain leucine-rich repeat-containing (NLR) proteins constitute an ancient class of intracellular immune receptors that confer innate immunity in plants and animals (Dodds and Rathjen, 2010; Jones et al., 2016). In plants, NLRs function by sensing pathogen-derived virulence molecules, known as effectors, and subsequently activating an immune response (Jacob et al., 2013; Kourelis and van der Hoorn, 2018). The majority of functionally validated NLRs in plants display broadly conserved domain architectures, typically consisting of the NB-ARC (nucleotide-binding adaptor shared by APAF-1, certain $R$ gene products and CED-4) domain, the LRR (leucin-rich repeat) region, and either a TIR (Toll/interleukin 1 receptor), CC (coiled-coil), or $C_{R}$ (RPW8-type CC) domain at the N-terminus (Kourelis and Kamoun, 2020; Shao et al., 2016). However, coevolution with pathogen effectors has led to a remarkable diversification of NLR repertoires, which form one of the most diverse protein families in plants (Lee and Chae, 2020; Prigozhin and Krasileva, 2020). An emerging paradigm in plant immunity is that some NLRs acquired novel recognition specificities through fusions of noncanonical integrated domains (IDs) that mediate perception of effectors (Cesari et al., 2014a; Wu et al., 2015). Although NLR-IDs have been described across various plant families (Gao et al., 2018; Kroj et al., 2016; Sarris et al., 2016; Van de Weyer et al., 2019), little is 
known about their emergence and subsequent evolution. In addition, our knowledge about how NLRs adapt to rapidly evolving pathogen effectors remains sparse.

Given that many IDs exhibit homology to molecules required for immune responses, they are generally thought to have derived from effector operative targets, which then act as baits for effector recognition within NLRs (Cesari et alo, 2014a; Wu et al., 2015). IDs can perceive effectors by direct binding, by serving as substrates for their enzymatic activities, or by detecting effectorinduced perturbations (Bao et al., 2017; Cesari et al., 2014a; Fujisaki et al., 2017; Heidrich et al., 2013; Sarris et al., 2016; Wu et al., 2015). The RGA5 (also known as Pia-2) and Pik-1 receptors are well-characterised examples of NLR-IDs. RGA5 and Pik-1 detect three unrelated effectors from the rice blast fungus, Magnaporthe oryzae, AVR-Pia/AVR1-CO39 and AVR-Pik, respectively, via their integrated heavy metal-associated (HMA) domains (De la Concepcion et al., 2018; Guo et al., 2018). HMAs are commonly found in a family of HMA plant proteins (HPPs) or HMA isoprenylated plant proteins (HIPPs) known to contribute to abiotic and biotic stress responses (de Abreu-Neto et alı, 2013; Fukuoka et al., 2009; Li et al., 2020a; Radakovic et alı, 2018; Zschiesche et al., 2015). Recently, the AVR-Pik effectors have been shown to bind and stabilise rice HMA proteins to co-opt their function in immunity (Maidment et al., 2020; Oikawa et al., 2020), providing direct evidence that integrated HMAs indeed mimic host targets of effectors.

NLR-triggered immunity is usually accompanied by the hypersensitive response (HR), a type of localised cell death associated with disease resistance. Notably, several NLR-IDs appear to have lost the ability to autonomously trigger a defence response (Cesari et al., 2014b; Zdrzałek et al., 2020). As a consequence, they often function in pairs, where the NLR-ID serves as a sensor for pathogen effectors and its partner acts as a helper that mediates activation of an immune response (Adachi et al., 2019; Bonardi et al., 2011; Feehan et al., 2020). There are now many examples of such NLR pairs, including RRS1/RPS4 from Arabidopsis thaliana (Saucet et al., 2015) as well as Pik1/Pik-2 (Ashikawa et alo, 2008), Pii-2/Pii-1 (Fujisaki et al., 2017), and RGA5/RGA4 (the Pia locus) (Cesari et al., 2014b; Okuyama et al., 2011) from rice. Many NLR pairs are encoded by two adjacent genes in a head-to-head orientation (Bailey et al., 2018; Van de Weyer et al., 2019). This genetic linkage likely provides an evolutionary advantage by facilitating co-segregation, coevolution, and transcriptional coregulation of functionally linked genes (Baggs et al., 2017; Griebel et alo, 2014). Genetic linkage may also reduce the genetic load caused by autoimmunity (Wu et al., 2018), which is a common phenomenon observed across NLRs (Alcázar et al., 2009; Bomblies et al., 2007; Chae et al., 2016; Deng et al., 2019; Yamamoto et al., 2010).

Rice Pik-1 and Pik-2 proteins form a CC-type NLR pair. Two Pik haplotypes, N- and K-type, are present in the genetic pool of wild and cultivated rice (Zhai et al., 2011). While the function of the $\mathrm{N}$-type haplotypes remains obscure, K-type Pik NLRs confer resistance to the rice blast fungus. In the K-type pair, Pik-1 acts as a sensor that binds the AVR-Pik effector via the Pik-1-integrated HMA domain, whereas Pik-2 is required for activation of immune response upon effector recognition (Maqbool et al., 2015; Zdrzałek et al., 2020). This NLR pair was initially cloned from Tsuyuake rice (Ashikawa et al., 2008) and has since been shown to occur in allelic variants, which include Pikp, Pikm, Piks, Pikh, and Pik* (Costanzo and Jia, 2010; Jia et al., 2009; Wang et al., 2009; Yuan et al., 2011; Zhai et al., 2011). Remarkably, the integrated HMA domain is the most sequence-diverse region among Pik-1 variants, consistent with the view that the receptor is under selection imposed by AVR-Pik (Białas et alı, 2018; Costanzo and Jia, 2010; De la Concepcion et alı, 2021; Zhai et al., 2014). Conversely, AVR-Pik alleles carry only five amino acid replacements, all of which map to regions located at the HMA-binding interface, indicating the adaptive nature of those polymorphisms (Longya et al., 2019). While the most ancient of the AVR-Pik allelic variants, AVR-PikD, is recognised by a wide range of Pik-1 proteins, the most recent variants, AVR-PikC and AVR-PikF, evade recognition by all known Pik-1 variants (Kanzaki et al., 2012; Longya et al., 2019). These recognition specificities are thought to reflect the ongoing arms race between rice and the rice blast fungus (Białas et alı, 2018; Kanzaki et al., 2012; Li et al., 2019) and have been linked to the effector-HMA binding affinity (De la Concepcion et al., 2021; De la Concepcion et al., 2018; Maqbool et al., 2015). Despite the wealth of knowledge about mechanisms governing effector recognition by the Pik-1-integrated HMA domain, we know little about its evolutionary history.

Evolutionary molecular biology can inform mechanistic understanding of protein function. After decades of parallel research, molecular evolution and mechanistic research are starting to be used in conjunction to unravel the molecular basis of protein function within an evolutionary framework 
(Delaux et al., 2019). One approach to investigate the biochemical drivers of adaptation is to reconstruct the evolutionary trajectories of proteins of interest (Dean and Thornton, 2007; Harms and Thornton, 2013; Thornton, 2004). Using phylogenetic techniques and algorithms for ancestral sequence reconstruction (ASR), it is now possible to statistically infer ancestral sequences, which can then be synthesised, expressed, and experimentally studied in the context of modern sequences (Ashkenazy et al., 2012; Cohen and Pupko, 2011; Pupko et al., 2000). In the field of plantmicrobe interactions, experimental analyses of resurrected ancestral effector sequences have helped unravel biochemical bases of effector specialisation and adaptive evolution following a host jump (Dong et al., 2014; Tanaka et al., 2019; Zess et al., 2019). To date, ancestral reconstruction has not been used to study the evolution of NLR immune receptors.

Despite remarkable advances in the field of NLR biology, there is still a significant gap in our understanding of how these receptors have adapted to fast-evolving pathogens. In this work, we used the rice Pik-1/Pik-2 system, coupled with ASR, to test hypotheses about adaptive evolution of NLRs and their IDs and to bridge the gap between mechanistic and evolutionary research. We leveraged the rich genetic diversity of the Pik genes in grasses and discovered that they likely derived from a single ancestral gene pair that emerged before the radiation of the major grass lineages. In addition, we show that the HMA integration predates speciation of Oryzinae dated at $\sim 15$ million years ago (MYA) (Jacquemin et al., 2011; Stein et al., 2018). Functional characterisation of a resurrected ancestral HMA (ancHMA), dating back to early Oryza evolution, revealed that different allelic variants of Pik-1, Pikp-1 and Pikm-1, convergently evolved from the weakly binding ancestral state towards high-affinity binding and recognition of the AVR-PikD effector through different biochemical paths. We conclude that for most of its evolutionary history Pik-HMA did not sense AVR-PikD and that recognition of this effector is a recent adaptation. This work provides new insights into our understanding of the dynamic nature of NLR adaptive evolution.

\section{Results}

\section{Pik orthologues are widely present across distantly related grass species}

To determine the diversity of the Pik-1 and Pik-2 genes across the Poaceae family (grasses), we performed a phylogenetic analysis of the entire repertoire of CC-NLRs from representative grass species. We used NLR-Parser (Steuernagel et al., 2015) to identify NLR sequences from publicly available protein databases of eight species (Supplementary file 1A). Following rigorous filtering steps (described in Materials and methods), we compiled a list of 3062 putative CC-NLRs (Supplementary file 2), amended with known and experimentally validated NLR-type proteins from grasses (Supplementary file 1B). Next, we constructed a maximum likelihood (ML) phylogenetic tree based on protein sequences of the NB-ARC domain of recovered CC-NLRs and discovered that the Pik-1 and Pik-2 sequences fell into two phylogenetically unrelated, but well-supported, clades (Figure 1-figure supplement 1A). Among Pik-1- and Pik-2-related sequences, we detected representatives from different, often distantly related, grass species, including members of the Pooideae and Panicoideae subfamilies. To determine the topologies within these clades, we performed additional phylogenetic analyses using codon-based sequence alignments of Pik-1 and Pik-2 clade members. Both Pik-1 and Pik-2 phylogenetic trees, calculated using the ML method, revealed the relationships within the two clades (Figure 1-figure supplement 1B). We propose that the identified clades consist of Pik-1 and Pik-2 orthologues from a diversity of grass species.

We noted that Pik-2 from Oryza brachyantha was N-terminally truncated as a result of a $46 \mathrm{bp}$ deletion within its $5^{\prime}$-region (Figure 1-figure supplement 2A). To determine whether the $O$. brachyantha population carries a full-length Pik-2 gene, we genotyped 16 additional $O$. brachyantha accessions (Figure 1-figure supplement 2B). We successfully amplified and sequenced six fulllength ObPik-2 genes, none of which carried the deletion present in the reference genome. We further amplified full-length ObPik-1 genes from the selected accessions (Supplementary file 1C), confirming that both full-length Pik-2 as well as Pik-1 are present in this species.

Following these results, we expanded the search of Pik orthologues to 10 additional species, focusing on members of the Oryzoideae subfamily (Supplementary file 1D). Using recurrent BLASTN searches combined with manual gene annotation and phylogenetic analyses, we identified 
A

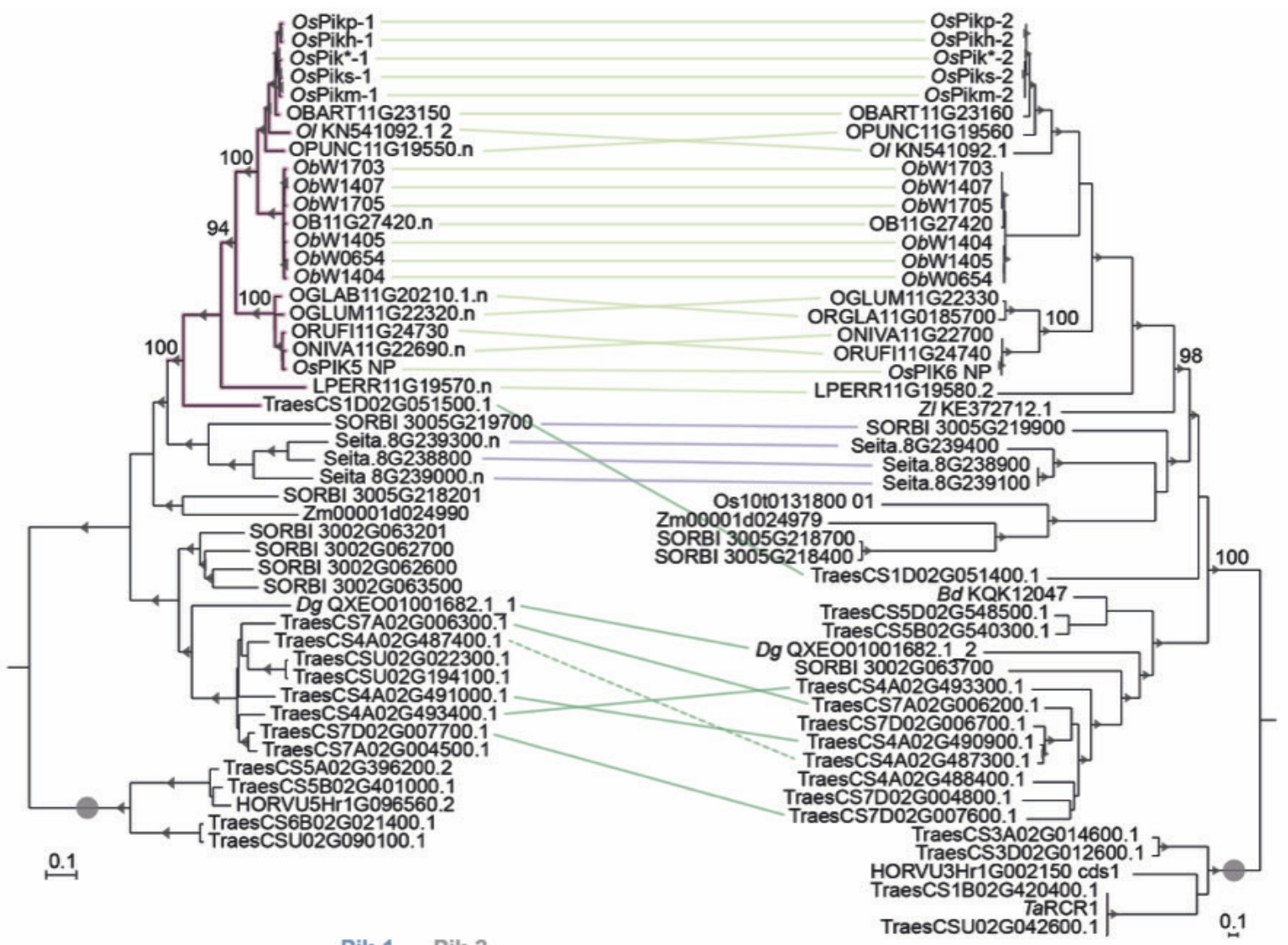

B

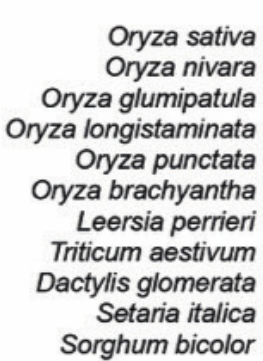

Pik-1 Pik-2
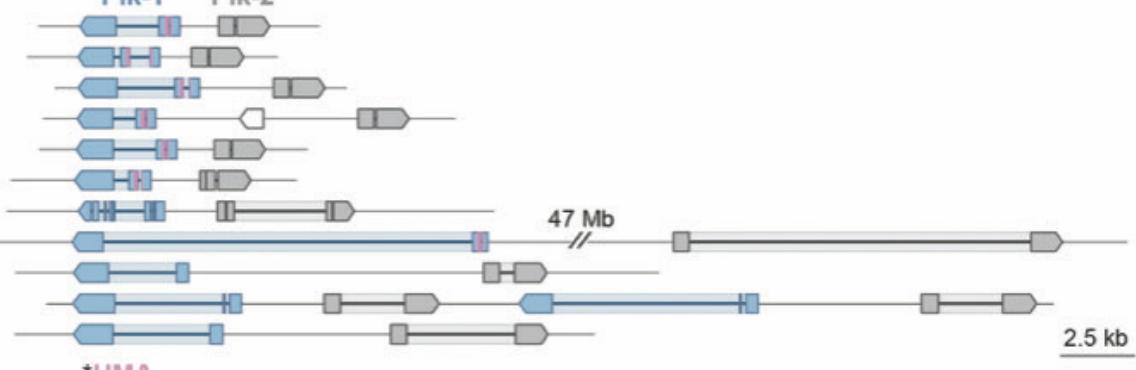

*HMA

C

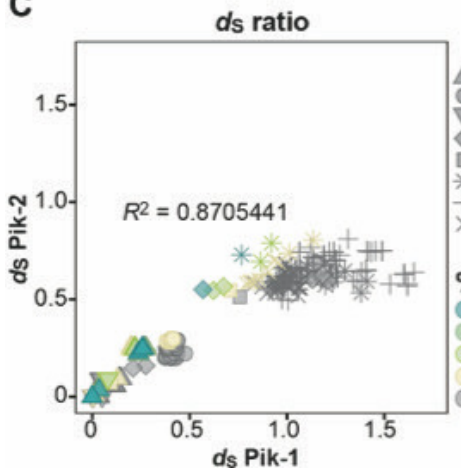

D

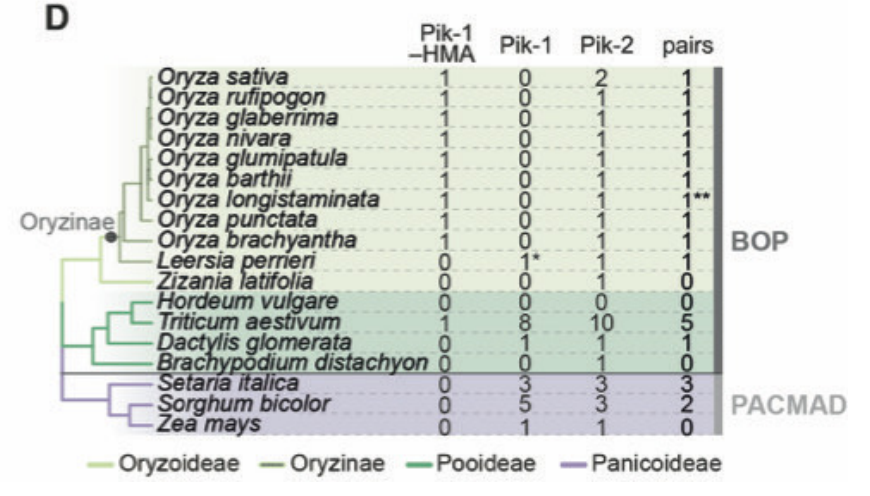

Figure 1. The Pik-1/Pik-2 orthologues are distributed across diverse species of grasses. (A) The maximum likelihood (ML) phylogenetic trees of Pik-1 (left) and Pik-2 (right) orthologues. The trees were calculated from 927- and 1239-nucleotide-long codon-based alignments of the NB-ARC domain, respectively, using RAxML v8.2.11 (Stamatakis, 2014), 1000 bootstrap method (Felsenstein, 1985), and GTRGAMMA substitution model (Tavaré, 1986). Best ML trees were manually rooted using the selected clades (marked with grey circles) as outgroups. The bootstrap values above Figure 1 continued on next page 
Figure 1 continued

$70 \%$ are indicated with grey triangles at the base of respective clades; the support values for the relevant nodes are depicted with numbers. The scale bars indicate the evolutionary distance based on nucleotide substitution rate. The Pik-1 integration clade is shown in pink. Genetically linked genes are linked with lines, with colours indicating plant subfamily: Oryzoideae (purple), Pooideae (dark green), or Panicoideae (light green); the continuous lines represent linkage in a head-to-head orientation, the dashed line indicates linkage in a tail-to-tail orientation. The interactive trees are publicly available at: https://itol.embl.de/tree/14915519290329341598279392 and https://itol.embl.de/tree/14915519290161451596745134. (B) Schematic illustration of the Pik locus in selected species. The schematic gene models of Pik-1 (blue) and Pik-2 (grey) are shown. The integrated heavy metal-associated (HMA) domain is marked with pink. The coordinates of the regions presented in this figure are summarised in Supplementary file 1E. (C) Comparisons of pairwise $d_{S}$ rates calculated for the Pik-1 and Pik-2 receptors. The rates were calculated using Yang and Nielsen, 2000 based on $972-$ and $1269-$ nucleotide-long codon-based alignments of the NB-ARC domains of Pik-1 and Pik-2, respectively; only positions that showed over 70\% coverage across the alignment were used for the analysis. The comparisons were categorised to reflect species divergence (shapes) and colour-coded to illustrate percentage identity of $d_{S}$ values (\% identity). The coefficient of determination $\left(R^{2}\right)$ was calculated for each dataset using $R$ v3.6.3 package. (D) Summary of identified Pik-1 and Pik-2 homologues in plant species included in this study. The phylogenetic tree was generated using TimeTree tool

(Kumar et al., 2017). The number of pairs correspond to the number of Pik-1/Pik-2 genes in a head-to-head orientation separated by intergenic region of various length. ${ }^{*}$ The species harbours a truncated gene between Pik-1 and Pik-2; ${ }^{*}$ the species has likely lost the HMA domain; Pik-1-HMA: Pik-1 with the HMA domain; Pik-1: Pik-1 without the HMA integration; BOP: Bambusoideae, Oryzoideae, Pooideae; PACMAD: Panicoideae, Arundinoideae,

Chloridoideae, Micrairoideae, Aristidoideae, Danthonioideae.

The online version of this article includes the following source data and figure supplement(s) for figure 1:

Source data 1. Selection test for Pik-1 vs. Pik-2 orthologues.

Figure supplement 1. Pik-1 and Pik-2 orthologues fall into two well-supported clades.

Figure supplement 2. Genotyping of Oryza brachyantha accession.

Figure supplement 3. Pik-1 and Pik-2 orthologues from Oryza spp. fall into K- and N-type clades.

Figure supplement 4. Schematic representation of selected Pik clusters in wheat (T. aestivum), sorghum (S. bicolor), and foxtail millet (S. italica).

Figure supplement 5. Random pairwise comparisons of $d_{S}$ rates calculated for the Pik-1 and Pik-2 receptors.

Figure supplement 6. Genetically linked Pik-1 and Pik-2 have similar molecular age.

additional Pik-related NLRs resulting in 41 and 44 Pik-1 and Pik-2 sequences, respectively (Figure 1A). Altogether, the additional Pik orthologues gave us a broad view of their occurrence in monocots. The majority of species within the Oryzinae subtribe contain single copies of Pik-1 and Pik-2 per accession, whereas members of the Pooideae and Panicoideae subfamilies frequently encode multiple Pik-1 or Pik-2 paralogues, with wheat carrying as many as 9 and 10 Pik-1 and Pik-2 genes, respectively. It is possible that ancestral Pik-1 and Pik-2 experienced a duplication before the radiation of Pooideae and Panicoideae followed by different patterns of gene loss/retention among grass species; however, a better-resolved phylogeny is needed to test this possibility. In addition, Pik-1 and Pik-2 from the Oryza genus formed two subclades, corresponding to the two haplotypes previously identified at the Pik locus, N-type and K-type (Figure 1-figure supplement 3; Zhai et al., 2011). We conclude that the $\mathrm{N}$ - and K-type Pik genes have been maintained through speciation and coexist as haplotypes in different Oryza species. Altogether, we discovered that Pik-1 and Pik-2 orthologues are present across a wide range of grasses, including members of the Oryzoideae, Pooideae, and Panicoideae subfamilies.

\section{Genetic linkage of the Pik gene pair predates the split of major grass lineages}

In rice, the Pikp-1 and Pikp-2 genes are located in a head-to-head orientation at a single locus of chromosome 11, and their coding sequences are separated by an $\sim 2.5$-kb-long region (Ashikawa et al., 2008; Yuan et al., 2011). To determine whether this genetic linkage is conserved in grasses, we examined the genetic loci of retrieved Pik-1 and Pik-2 genes. A total of 14 out of 15 species in which both genes are present carry at least one Pik pair with adjacent Pik-1 and Pik-2 genes in a head-to-head orientation. Although the length of the genes and their intergenic regions vary between species (from $\sim 2 \mathrm{~kb}$ in $\mathrm{O}$. nivara to $\sim 48 \mathrm{Mb}$ in wheat), they exhibit largely conserved gene models. Most of the Pik-2 orthologues feature one intron in their nucleotide-binding domain (NBD) region (Ashikawa et al., 2008) while the Pik-1 genes typically carry one or, for the genes featuring the HMA domain, two introns (Figure 1B; Supplementary file 1F). In addition, in species that carry multiple copies of Pik-1 or Pik-2, the copies are typically located in close proximity or, as in wheat, in large NLR-rich gene clusters (Figure 1-figure supplement 4; Supplementary file 1F). 
Given that genomic rearrangements have been reported at the Pik locus (Mizuno et al., 2020; Stein et al., 2018), we could not exclude the possibility that genetic linkage of the Pik-1/Pik-2 pair emerged more than once and is a remnant of rearrangement events. We reasoned that if the gene pair have remained genetically linked over a long evolutionary period, then they should have the same molecular age. To gain insights into the evolutionary dynamics between genetically linked Pik1 and Pik-2 receptors, we compared their rates of synonymous substitutions $\left(d_{\mathrm{S}}\right)$. For this analysis, we selected representative Pik-1 and Pik-2 NLRs that are genetically linked in a head-to-head orientation from 13 species; LpPik (Leersia perrieri) orthologues were excluded from the analysis because their unusual gene models interfered with sequence alignments (Figure 1B). Next, we assessed $d_{S}$ within the coding sequences of the NB-ARC domain between pairwise genes using the Yang and Nielsen, 2000 method. The rates were calculated separately for Pik-1 and Pik-2 and cross-referenced such that the pairwise values for Pik-1 were compared to the respective values for cognate Pik-2 (Figure 1-source data 1). The comparisons revealed strong positive correlation of $d_{s}$ rates $\left(R^{2}=0.87, \mathrm{p}\right.$-value=0.95) between genetically linked Pik genes (Figure $\left.1 C\right)$. This was significantly higher than observed by chance, as calculated from random Pik-1-Pik-2 cross-referencing (Figure 1figure supplement 5). We conclude that the Pik-1/Pik-2 pair probably became genetically linked long before the emergence of the Oryzinae clade and prior to the split of the major grass lineagesthe BOP (for Bambusoideae, Oryzoideae, Pooideae) and PACMAD (for Panicoideae, Arundinoideae, Chloridoideae, Micrairoideae, Aristidoideae, Danthonioideae) clades-which dates back to 100-50 MYA (Hodkinson, 2018).

\section{The HMA integration of Pik-1 predates the emergence of Oryzinae}

To better understand the evolutionary history of Pik-1 domain architecture, we looked for signatures of HMA integration among the collection of 41 Pik-1 orthologues identified. Remarkably, the presence of an HMA domain varied among Pik-1 genes. HMA-containing Pik-1 clustered into a single well-supported clade (herein called the Pik-1 integration clade) (Figure 1A). All members of the Pik1 integration clade carry the HMA domain in the same position, between the CC and NB-ARC domains of Pik-1, and feature an intron within the HMA (Figure 1B). This implies that these HMA domains are likely derived from a single integration event.

Using this information, we generated a sequence alignment of selected Pik-1 orthologues to define the position of the HMA integration (Figure 2-figure supplement 1). We focused on comparisons of representative members of the Pik-1 integration clade and their closest relatives from Setaria italica and Sorghum bicolor. This revealed that the integration site most likely falls between the KLL and KTV residues (corresponding to residues 161-163 and 284-286 of Pikp-1); however, the exact boundaries of the integration might be slightly different, given the relatively high sequence divergence around this site among the more distantly related orthologues. We further noted that the integration site encompasses a wider region than that of functionally characterised HMA domains (De la Concepcion et al., 2021; De la Concepcion et al., 2018), with around 20 additional amino acids (23 and 21 in Pikp-1) on each side of the annotated HMA domain.

Next, we estimated when Pik-1 acquired the HMA from the phylogeny of the plant species with Pik-1 orthologues (Figure 1D). We found that all Oryza Pik-1 orthologues carry the HMA domain, which indicates that the integration predates speciation of this genus. Although we failed to detect a full-length HMA integration in L. perrieri, LpPik-1 carries 15 amino acids characteristic of the HMA integration site (Figure 2-figure supplement 1), indicating that the fusion probably occurred before the speciation of Oryzinae, dated at 15 MYA (Jacquemin et al., 2011), and was subsequently lost in L. perrieri. By contrast, the vast majority of examined Pik-1 from the Pooideae and Panicoideae subfamilies lack the HMA domain. The only integration in these taxonomic groups was detected in one of the nine Pik-1 paralogues of wheat included in the analysis. This observation may indicate that the Pik-1-HMA fusion may have emerged prior to radiation of the BOP clade, 100-50 MYA (Hodkinson, 2018). However, it is also possible that the integration occurred much later and that the newly emerged Pik-1-HMA gene transferred to wheat through introgression from rice progenitors. In summary, we can confidently conclude that the HMA integration of Pik-1 predates the emergence of the Oryzinae. 


\section{The integrated HMA domain carries signatures of positive selection}

In rice, the Pik-1-integrated HMA domain exhibits higher levels of polymorphisms compared with canonical domains of Pik-1 and Pik-2 (Costanzo and Jia, 2010; Kanzaki et al., 2012). To characterise the pressures underlying HMA diversification, we examined molecular signatures of selection within the Pik-1 integration clade. Wheat Pik-1-HMA was excluded from the analysis due to its high sequence divergence relative to Oryza orthologues, which precluded generating reliable sequence alignments. For the same reason, the remaining sequences were assigned into $\mathrm{K}$ - and $\mathrm{N}$-type sequences based on phylogenetic relationship and analysed separately. To test for signatures of selection, we calculated rates of synonymous $\left(d_{S}\right)$ and nonsynonymous $\left(d_{N}\right)$ substitutions across the coding sequences of the HMA domain. We discovered that $d_{N}$ was greater than $d_{S}$ in 96 out of 115 pairwise sequence comparisons (86/105 for $\mathrm{K}$ - and 10/10 for $\mathrm{N}$-type HMAs; $\omega=d_{\mathrm{N}} / d_{\mathrm{S}}$ ranging 0 2.45 for K-type and 1.13-3.50 for $\mathrm{N}$-type) (Figure 2A-C), providing evidence that positive selection has acted on the integrated HMA domain. By contrast, only 9 out of 115 pairs of the NB-ARC domain sequences of the same set of genes displayed $d_{N}$ greater than $d_{S}$ (Figure 2B-D); however, all of these showed $d_{S}=0$ and were therefore inconclusive in calculating $\omega\left(d_{N} / d_{S}\right)$ ratios. A comparison of the $d_{N}$ and $d_{S}$ rates between the HMA and NB-ARC domains further highlighted the elevated rates of nonsynonymous substitutions within the integrated HMA domain relative to NB-ARC (Figure 2-figure supplement 2). Overall, these results demonstrate that the integrated HMA domain exhibits marked signatures of positive selection in contrast to the Pik-1 NB-ARC domain.

Positive selection typically acts only on particular amino acids within a protein. Therefore, we aimed to detect sites within the integrated HMA domain that experienced positive selection using the ML method (Yang et al., 2000). To capture additional Pik-1-integrated HMAs, we first genotyped further wild rice species for the presence of the integration. We detected the HMA integration in 21 accessions from 13 species (Supplementary file 1H); 10 of those showed sufficient coverage across the entire functional region of the HMA and were used for further analysis (Figure 2-figure supplement 1, Figure 2-figure supplement 3A). We excluded the $\mathrm{N}$-type HMA domains from the dataset owing to their small sample size $(n=5)$, which would prevent meaningful data interpretation. To detect patterns of selection within the K-type integrated HMA, we applied three pairs of ML models of codon substitution: M3/M0, M2/M1, and M8/M7 (Yang et al., 2000). As indicated by the likelihood ratio tests (LRTs) and posterior probabilities, $26 \%$ of the HMA amino acid sites likely experienced positive selection (Figure 2-figure supplement 3B, C; Supplementary file 3). As a control, we performed the same tests on the NB-ARC domain of the K-type Pik-1 sequences. Although the discrete M3 model inferred that a subset of NB-ARC amino acids might be under diversifying selection (Figure 2-figure supplement 4), other tests failed to detect patterns of positive selection. Based on these results, we conclude that the HMA domain exhibits strong signatures of positive selection compared with the NB-ARC domain.

\section{Ancestral sequence reconstruction of the Pikp-1-integrated HMA domain}

To understand the evolutionary trajectory of the Pik-1-integrated HMA domain, we used representative phylogenetic trees of the K-type HMA domains to reconstruct ancHMA sequences dating to the early stages of Oryza genus speciation. As an outgroup, we selected HMA sequences of the integrated HMA progenitors, HPPs and HIPPs (de Abreu-Neto et alı, 2013; Oikawa et alo, 2020), hereafter called non-integrated HMAs, from $O$. sativa and $O$. brachyantha. To perform the reconstruction, we first tested different phylogenetic methods and focused on nodes that are wellsupported in both the neighbour joining (NJ) and ML phylogenies generated from a codon-based alignment (Figure 3-figure supplement 1). Next, we performed the ancestral sequence prediction based on protein sequence alignment, using FastML software (Ashkenazy et al., 2012), which has been previously shown to infer ancestral sequences with high accuracy (Randall et alo, 2016). Multiple reconstructions yielded multiple plausible ancHMA variants (Figure 3-figure supplement 2; Supplementary file 4). To reduce the possibility of incorrect prediction, we selected six representative well-supported sequences for further studies. 
A

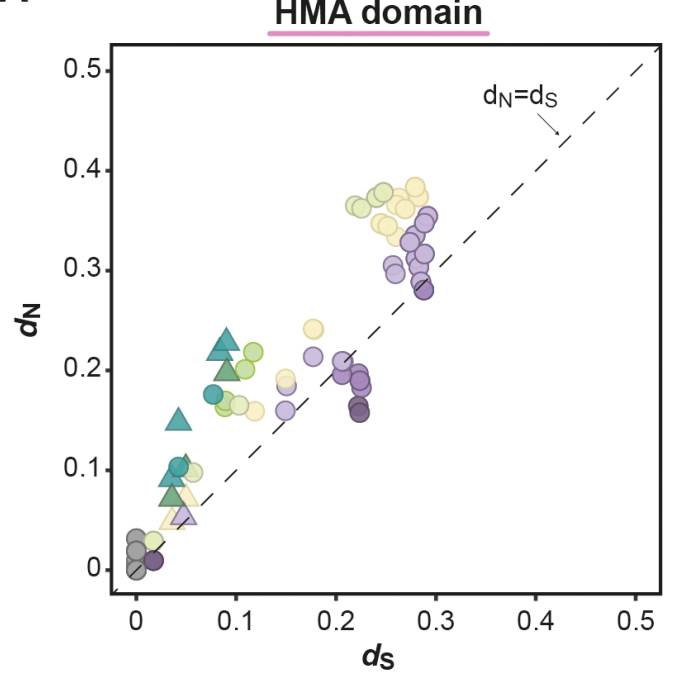

C

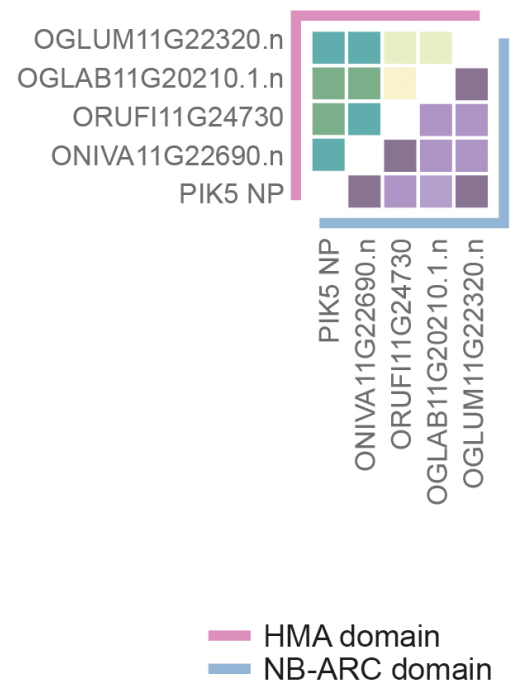

B

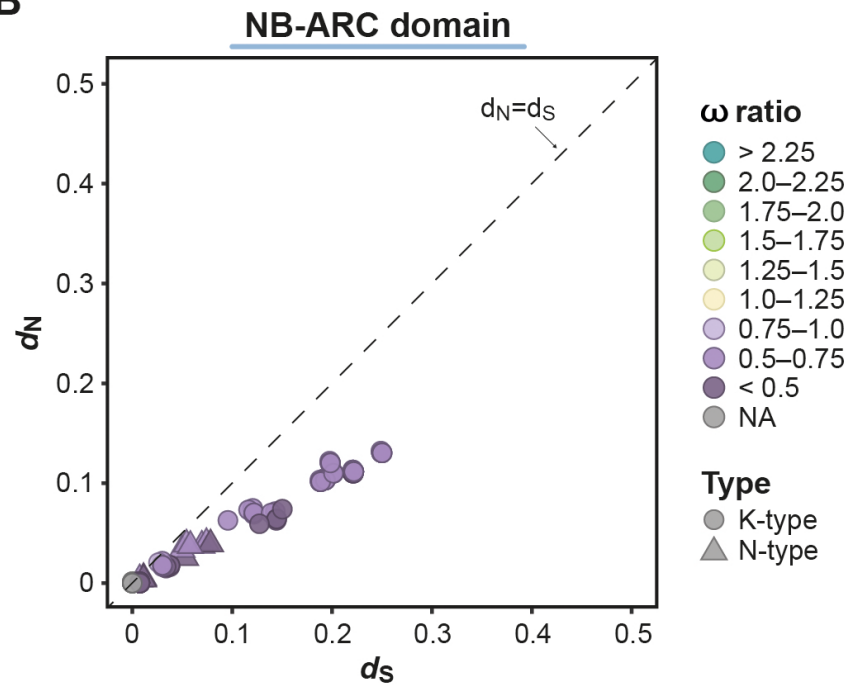

D

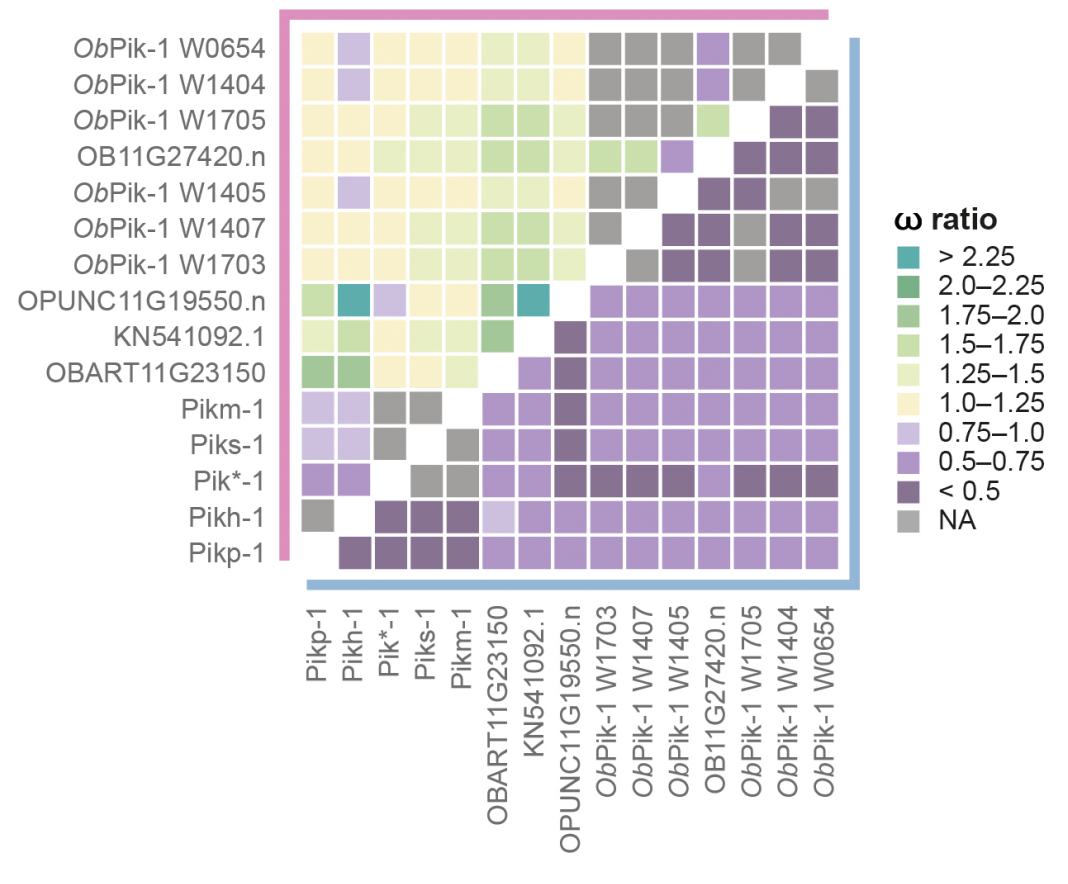

Figure 2. The integrated heavy metal-associated (HMA) domain exhibits elevated rates of $\omega\left(d_{N} / d_{S}\right)$ compared with the NB-ARC domain of Pik-1. (A, B) Pairwise comparison of nucleotide substitution rates within the Pik-1 integration clade for the (A) HMA and (B) NB-ARC domains, calculated using Yang and Nielsen, 2000. The diagonal line (dashed) indicates $d_{N}=d_{s}$. The points are colour-coded to indicate $\omega$ ratio; NA: the ratio was not calculated because $d_{S}=0$. The pairwise comparisons were separately performed for the K-type (circles) and N-type (triangles) Pik-1 sequences. (C, D) To highlight the differences between the $\omega$ rates for the HMA (pink line) and NB-ARC (blue line) domains, the rates were plotted as heatmaps corresponding to the (C) N- and (D) K-type Pik-1 sequences.

The online version of this article includes the following source data and figure supplement(s) for figure 2:

Source data 1. Selection test for Pik-1-HMA vs. NB-ARC.

Figure supplement 1. Multiple sequence alignment illustrating the conservation around the HMA integration site.

Figure supplement 2. The integrated heavy metal-associated (HMA) domain displays elevated rates of $d_{N}$ compared with the NB-ARC domain of Pik1.

Figure supplement 3. Residues within the integrated heavy metal-associated (HMA) domain are likely to have experienced positive selection.

Figure supplement 4. Selection test at the amino acid sites within the NB-ARC domain of the K-type Pik-1 genes. 


\section{Reconstructed ancHMAs exhibit weaker association with AVR-PikD compared to modern Pikp-HMA}

As high-affinity binding to the effector is required for the Pik-mediated immune response (De la Concepcion et alo, 2021; De la Concepcion et alo, 2019; De la Concepcion et al., 2018; Maqbool et al., 2015), we hypothesised that the HMA domain of Pikp-1 (Pikp-HMA) evolved towards high-affinity binding to AVR-PikD-the most ancient of the AVR-Pik effector alleles (Bentham et alo, 2021; Kanzaki et al., 2012). To test this hypothesis, we resurrected the six ancHMA variants determined above by synthesising their predicted sequences and incorporating them into the Pikp-1 receptor, generating Pikp-1:I-N2, Pikp-1:I-N6, Pikp-1:II-N11, Pikp-1:II-N12, Pikp-1:III-N11, and Pikp-1:III-N12 fusions (Figure 3A). We then tested their association with AVR$\mathrm{PikD}$ in planta in co-immunoprecipitation (co-IP) experiments. The western blot analysis revealed that the ancHMA variants exhibited a range of association strengths with AVR-PikD (Figure 3B; Figure 3-figure supplement 3). In every case, the association with ancHMA proteins was weaker than with the present-day Pikp-HMA, indicating that binding strength has likely changed over the course of the Pikp-HMA evolutionary history. For further studies, we selected the I-N2 ancHMA variant-the last common ancestor of Pik ${ }^{*}-1$, Pikp-1, Pikh-1, Piks-1, and Pikm-1-that was predicted with high

A

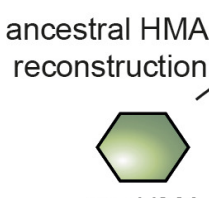
ancHMA resurrection by

replacing Pikp-HMA with ancHMA

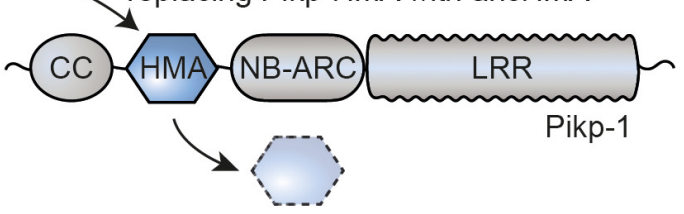

Pikp-HMA

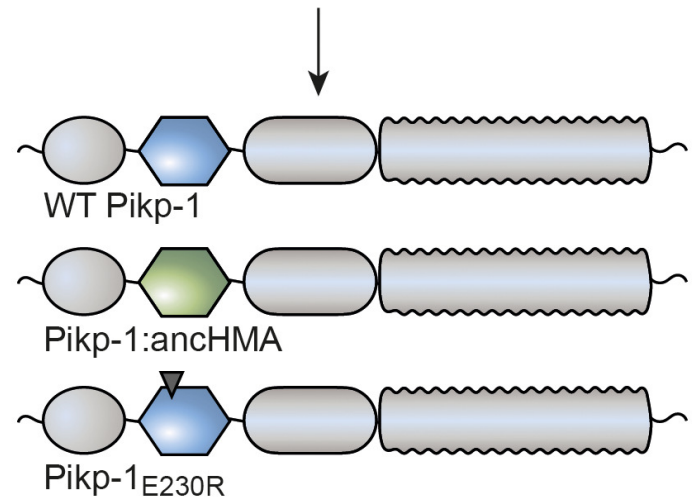

B

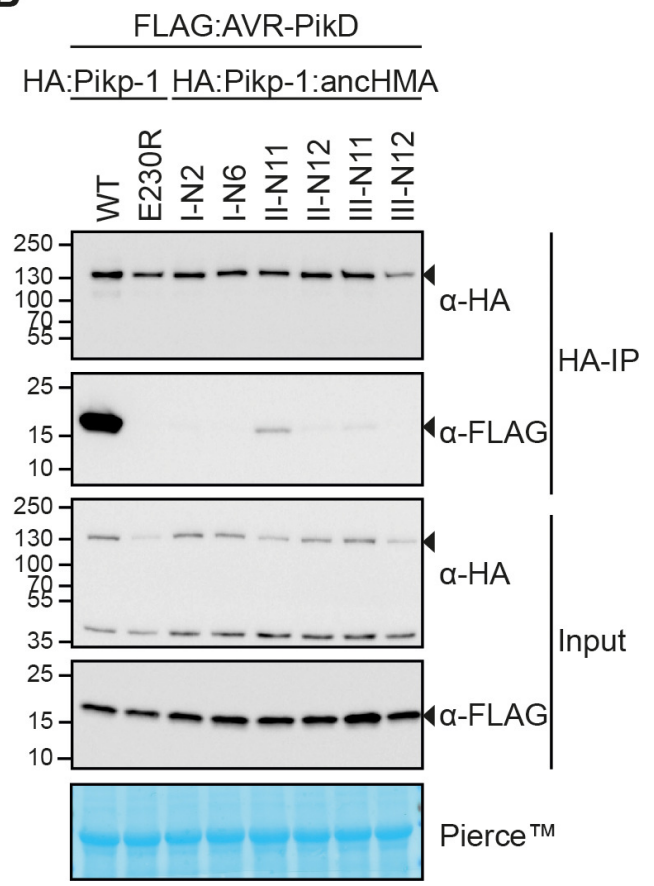

Figure 3. The integrated heavy metal-associated (HMA) domain of Pikp-1 exhibits stronger association with the AVR-PikD effector than its predicted ancestral state. (A) Overview of the strategy for resurrection of the ancestral HMA (ancHMA) domain. Following ancestral sequence reconstruction, the gene sequences were synthesised and incorporated into Pikp-1 by replacing the present-day Pikp-HMA domain (blue) with the ancHMA equivalent (green). (B) Co-immunoprecipitation experiment between AVR-PikD (N-terminally tagged with FLAG) and Pikp-1 (N-terminally tagged with HA) carrying ancestral sequences of the HMA. Wild-type (WT) HA:Pikp-1 and HA:Pikp-1 E230R were used as a positive and negative control, respectively.

Immunoprecipitates (HA-IP) obtained with anti-HA probe and total protein extracts (Input) were immunoblotted with appropriate antisera (listed on the right). Rubisco loading control was performed using Pierce staining solution. Arrowheads indicate expected band sizes. Results from three independent replicates of this experiment are shown in Figure 3-figure supplement 3.

The online version of this article includes the following figure supplement(s) for figure 3 :

Figure supplement 1. Phylogenetic analyses of the heavy metal-associated (HMA) domain of K-type Pik-1 NLRs.

Figure supplement 2. Ancestral sequence reconstruction yielded multiple plausible ancestral HMA (ancHMA) sequences.

Figure supplement 3. Replicates of the co-immunoprecipitation (co-IP) experiment between AVR-PikD and the reconstructed ancestral HMA (ancHMA) sequences. 
confidence in probability-based method (Supplementary file 4) and manual accuracy assessment. The variant is called ancHMA hereafter.

\section{The IAQVV/LVKIE region of the Pikp-HMA domain determines high- affinity AVR-PikD binding}

Next, we aimed to investigate which of the structural regions in the HMA encompass adaptive mutations towards AVR-PikD binding. By combining sequence and structural information available for Pikp-HMA (De la Concepcion et al., 2018; Maqbool et al., 2015), we identified four polymorphic regions between the ancestral and modern Pikp-HMA (Figure 4A, B). We sequentially replaced each of these regions in Pikp-1:ancHMA with the corresponding region from Pikp-HMA. Altogether, we obtained a suite of four chimeric HMAs-ancHMA $A_{A M E G N N D}$, ancHMA $A_{L V K I E}$, ancHMA $A_{L Y}$, ancHMA and assayed these for gain-of-binding to AVR-PikD in planta in co-IP experiments. Among tested constructs, only the Pikp-1:ancHMA Pikp-1 (Figure 4C, Figure 4-figure supplement 1). This indicates that the polymorphic residues in the IAQVV/LVKIE region are critical for the evolution of enhanced AVR-PikD binding in Pikp-1.

\section{Two substitutions within the IAQVV/LVKIE region of ancHMA increase binding to AVR-PikD}

To understand the evolutionary trajectory of the IAQVV/LVKIE region, we set out to reconstruct the evolutionary history of this region. We performed probability-based ASR, based on protein

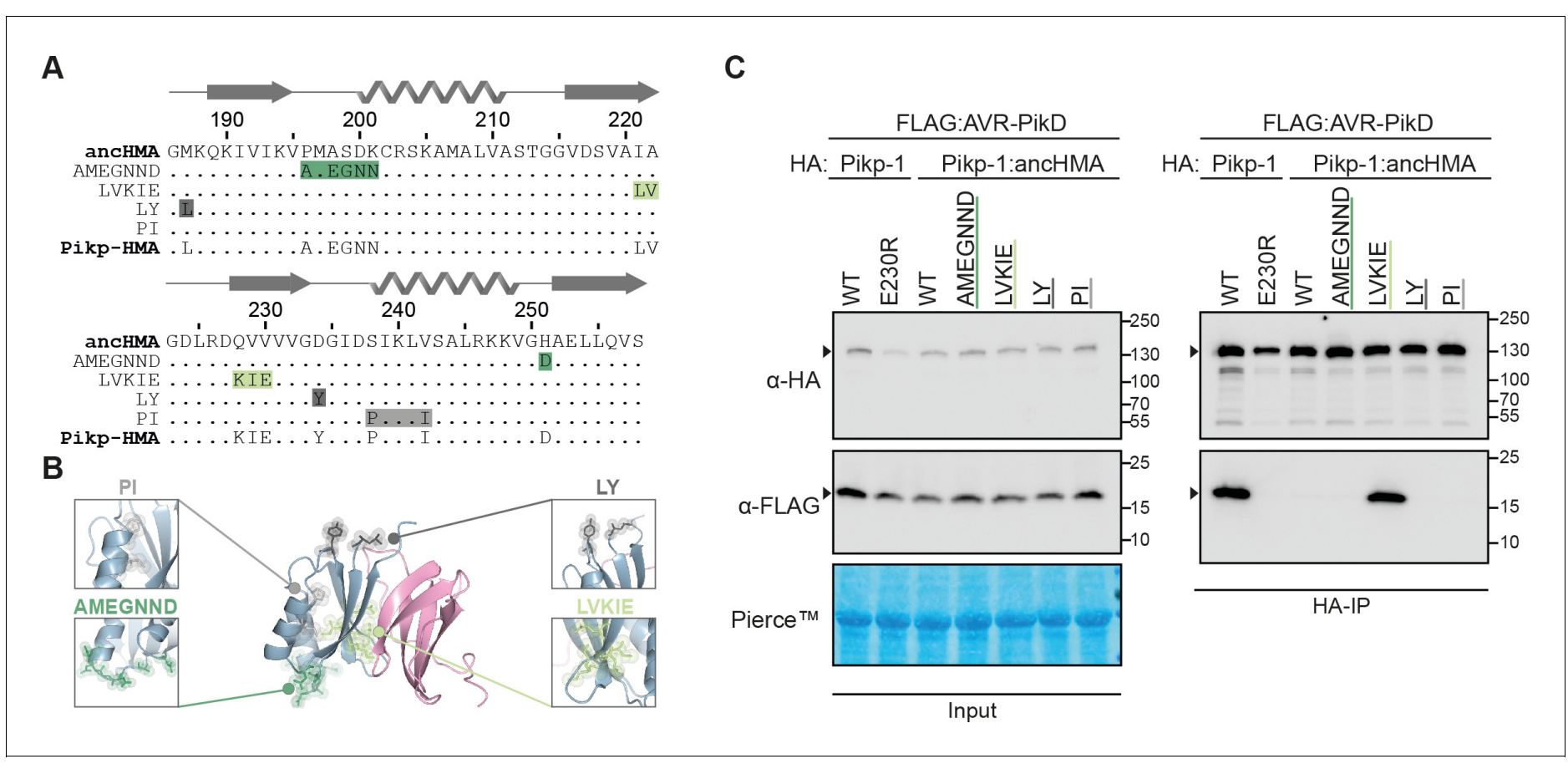

Figure 4. The IAOVV/LVKIE region of the Pikp-HMA domain determines high-affinity binding to AVR-PikD. (A) Protein sequence alignment showing the Pikp-ancHMA swap chimeras. The amino acid sequences of ancestral HMA (ancHMA), Pikp-HMA, and chimeras are aligned, with the protein model above corresponding to the Pikp-HMA structure. The colour-coded rectangles correspond to polymorphic regions used for chimeric swaps. (B) Schematic representation of Pikp-HMA (blue) in complex with AVR-PikD (pink) (De la Concepcion et alı, 2018), with polymorphic regions between the Pikp-HMA and the ancHMA colour-coded as in (A). The molecular surfaces of the polymorphic residues are also shown. (C) Association between AVRPikD (N-terminally tagged with FLAG) and Pikp-1, Pikp-1 E230R, Pikp-1:ancHMA, and Pikp-1:ancHMA chimeras (N-terminally tagged with HA), labelled above, was tested in planta in co-IP experiment. Wild-type (WT) Pikp-1 and Pikp-1 E230R were used as a positive and negative control, respectively. Immunoprecipitates (HA-IP) obtained with anti-HA probe and total protein extracts (input) were immunoblotted with the appropriate antisera, labelled on the left. Rubisco loading control was performed using Pierce staining solution. Arrowheads indicate expected band sizes. Results from three independent replicates of this experiment are shown in Figure 4-figure supplement 1.

The online version of this article includes the following figure supplement(s) for figure 4 :

Figure supplement 1. Replicates of the co-immunoprecipitation (co-IP) experiment between AVR-PikD and the Pikp-1:ancHMA chimeras. 
sequence alignment and a representative phylogeny of 19 K-type integrated HMA domains, where ancHMA was separated from Pikp-HMA by five internal nodes (Figure 3-figure supplement 2). Using sequences predicted at these nodes, we identified the three most ancient substitutions at the resolution of single amino acids-1221L, followed by Q228L, followed by V229E (Figure 5A). Discerning the order of the two most recent substitutions, Ala-222-Val and Val-230-Glu, was not possible. We generated ancHMA mutants by consecutively introducing historical substitutions into their respective ancestral backgrounds, generating ancHMA $A_{L A O V V}$, ancHMA $A_{L A K V V}$, and ancHMA $A_{L A K I V}$, as well as two plausible alternative states between LAKIV and LVKIE-ancHMA LAKIE and ancHMA $A_{\text {LVKIV. }}$

To determine the extent to which each of the historical mutations contributed to changes in effector binding, we cloned the ancHMA mutants into the Pikp-1 background and assayed them for AVRPikD binding in planta. Initial results showed low accumulation levels of Pikp-1:ancHMA preventing meaningful interpretation of results obtained using this protein (Figure 5-figure supplement 1), hence, we excluded it from further analysis; the remaining constructs accumulated to simi-

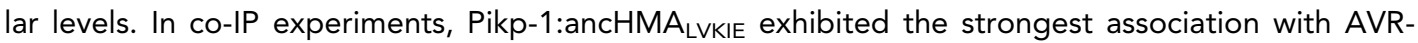
PikD followed by Pikp-1:ancHMA ${ }_{L A K I E}$, which displayed intermediate binding (Figure 5B, Figure 5figure supplement 2). The remaining mutants did not show gain-of-binding to AVR-PikD when compared to Pikp-1:ancHMA.

To quantify how historical substitutions in the IAQVV/LVKIE region contributed to enhancing AVR-PikD binding, we carried out surface plasmon resonance (SPR) experiments using AVR-PikD and the full set of the ancHMA mutants cloned to match the residues Gly-186-Ser-258 of the full-length Pikp-1, which have previously been successfully used in vitro (Maqbool et al., 2015), purified from Escherichia coli by a two-step purification method (Figure 5-figure supplement 3). We measured binding by monitoring the relative response following AVR-PikD immobilisation on the NTA-sensor chip and injection of the ancHMA proteins at three different concentrations. To capture the binding dynamics, we recorded the response at two timepoints: at the end of HMA injection ('binding') and $15 \mathrm{~s}$ post-injection ('dissociation') (Figure 5-figure supplement 4A). We normalised the response units to the theoretical maximum response $\left(R_{\max }\right)$ and expressed the results as a percentage of $R_{\max }$ $\left(\% \mathrm{R}_{\max }\right)$, which gave a relative indication of binding strength. Average $\Delta \% \mathrm{R}_{\max }$, calculated from a difference between $R_{\max }$ for 'binding' and 'dissociation', was used as an off-rate approximate. AnCHMA $_{\text {LVKIE }}$ formed the strongest interaction with AVR-PikD at levels similar to Pikp-HMA, followed by ancHMA $A_{L A K I E}$, then ancHMA $A_{L A O V}$, ancHMA $A_{L A K I V}$, and ancHMA, which showed weaker interactions; we did not record any significant binding for ancHMA ure supplement 4B; Supplementary file 1I). These results indicate that the two most recent mutations, Ala-222-Val and Val-230-Glu, collectively referred to as AV-VE, determined HMA transition towards high-affinity AVR-PikD binding.

We noted from the panel of 19 integrated HMA sequences collected in this study that the AV-VE polymorphisms are unique to Pikp-1 and Pikh-1 of rice. The Pikp-1 and Pikh-1 genes are highly similar to each other; out of a total of three polymorphisms, there is only one synonymous substitution that distinguishes their nearly 3500-bp-long coding sequences (Supplementary file 1J). Although this precludes a rigorous estimation of evolutionary divergence times of the integrated HMAs, the near-absence of synonymous nucleotide polymorphisms between Pikp-1 and Pikh-1 suggests a very recent emergence of the AV-VE polymorphisms.

\section{The AV-VE substitutions are sufficient to increase binding affinity towards AVR-PikD}

To investigate the role of historical contingency in the evolutionary history of the Pikp-1-integrated HMA domain, we tested the impact of early historical substitutions from the ancestral IAOVV residues to the Pikp-1 LVKIE on effector-binding strength. We bypassed the historical sequence by incorporating the AV-VE mutations directly into ancHMA, generating Pikp:ancHMA $A_{I V O V E}$, and examined effector binding in co-IP experiments (Figure 5-figure supplement 5). Pikp:ancHMA showed stronger association with AVR-PikD than Pikp:ancHMA; however, we were unable to directly compare its association to Pikp:ancHMA $A_{\text {LVKIE }}$ due to uneven protein accumulation levels. These results indicate that the $A V-V E$ substitutions are sufficient to increase binding affinity towards the AVR-PikD effector independently of the other three polymorphic residues in this IAQVV/LVKIE interface. Nonetheless, we cannot exclude the possibility that prior mutations had quantitative epistatic effects on the interaction that cannot be quantified by co-IP. 
A

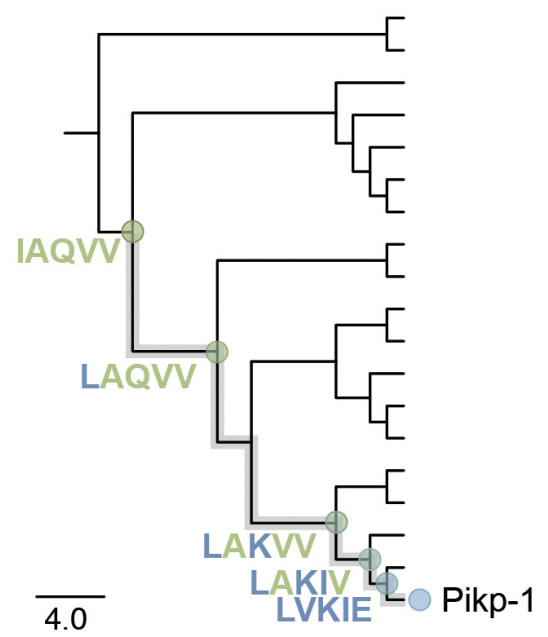

B

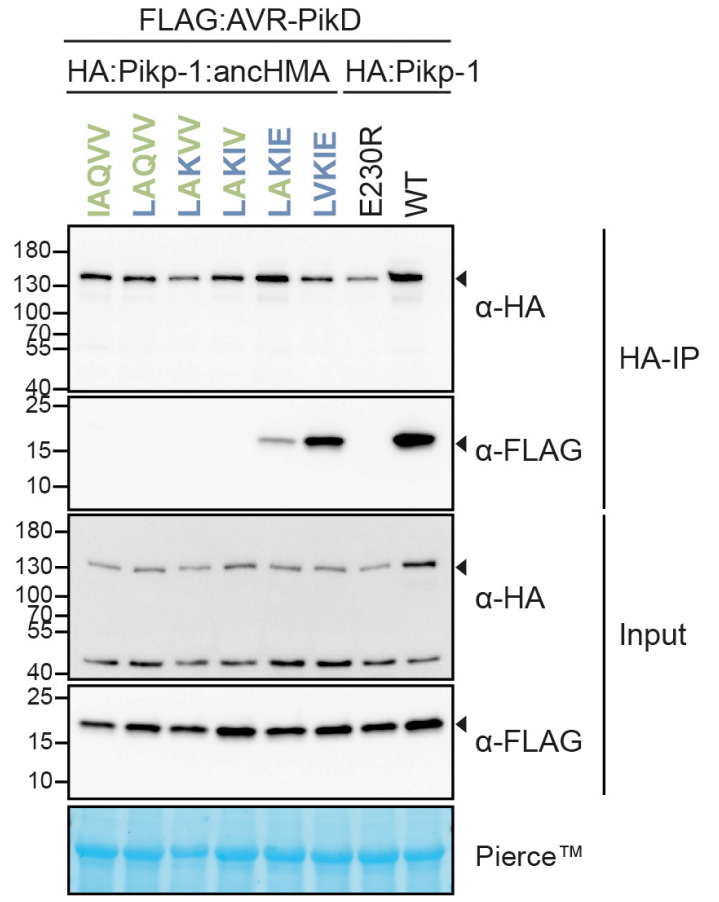

C
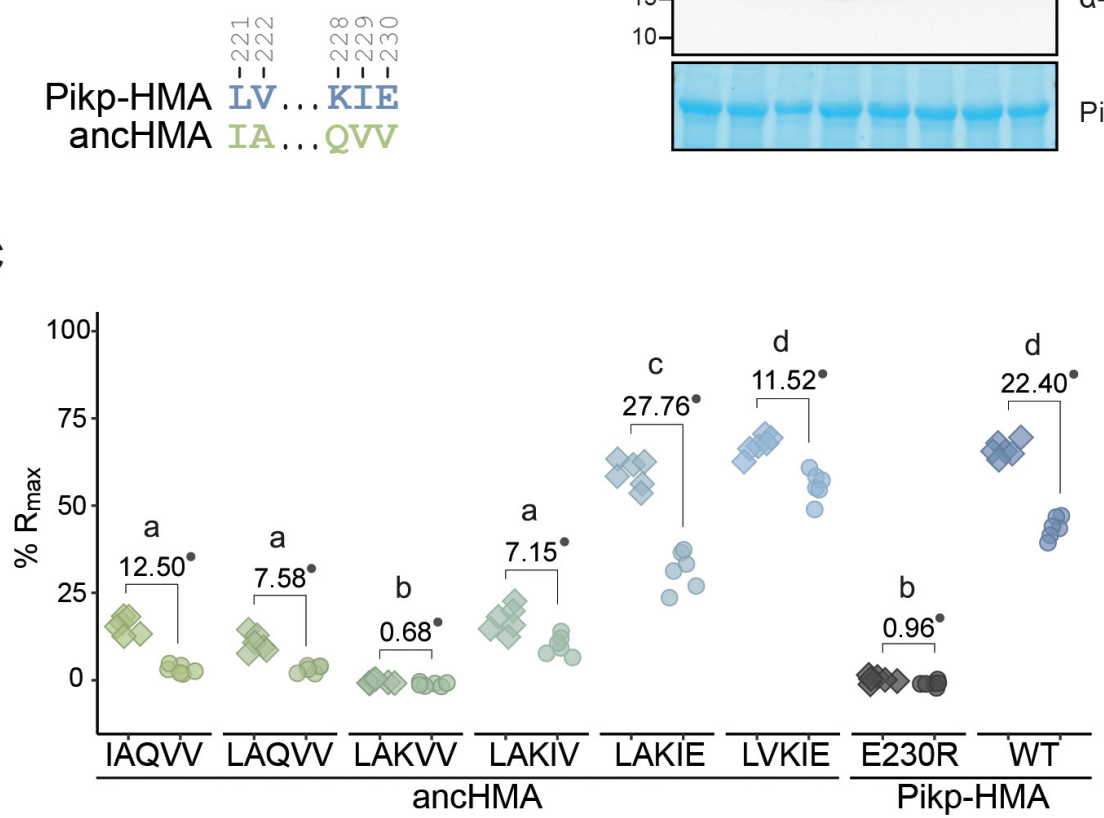

$\% \mathrm{R}_{\max }$ type: $\bullet$ binding $\bullet$ dissociation

-Average $\Delta \% R_{\max }$

Figure 5. The AV-VE substitutions within the IAOVV/LVKIE region of ancestral HMA (ancHMA) increase binding to AVR-PikD. (A) Schematic representation of a neighbour joining (NJ) phylogenetic tree of the heavy metalassociated (HMA) domain from Oryza spp. (shown in Figure 3-figure supplement 2). The scale bar indicates the evolutionary distance based on the number of base substitutions per site. Historical mutations in the IAOVV/LVKIE region acquired over the course of Pikp-HMA evolution are shown next to the appropriate nodes. The mutations are colour-coded to match the ancestral (green) and present-day (blue) states. (B) Co-immunoprecipitation (Co-IP) experiment illustrating in planta association of AVR-PikD (N-terminally tagged with FLAG) with Pikp-1 and Pikp-1: ancHMA (N-terminally tagged with HA), labelled above. Wild-type (WT) HA:Pikp-1 and HA:Pikp-1 E230R proteins were used as a positive and negative control, respectively. Immunoprecipitates (HA-IP) obtained with anti-HA probe and total protein extracts (input) were immunoblotted with appropriate antibodies (listed on the right). Loading control, featuring Rubisco, was performed using Pierce staining. The arrowheads indicate expected band sizes. Three independent replicates of this experiment are shown in Figure 5-figure supplement 2. (C) Plot illustrating calculated percentage of the theoretical maximum response $\left(\% R_{\max }\right)$ values for interaction of HMA Figure 5 continued on next page 
Figure 5 continued

analytes, labelled below, with AVR-PikD ligand (featuring C-terminal HIS tag) determined using surface plasmon resonance. \% $\mathrm{R}_{\max }$ was normalised for the amount of ligand immobilised on the NTA-sensor chip. The chart summarises the results obtained for HMA analytes at $400 \mathrm{nM}$ concentration from three independent experiments with two internal repeats. Three different concentrations of the analytes (400 nM, $200 \mathrm{nM}, 50 \mathrm{nM})$ were tested; results for the $200 \mathrm{nM}$ and $50 \mathrm{nM}$ concentrations are shown in Figure 5-figure supplement 4. Average $\Delta \% \mathrm{R}_{\max }$ (•) values represent absolute differences between values for 'binding' and 'dissociation', calculated from the average values for each sample, and serve as an off-rate approximate. Statistical differences among the samples were analysed with Tukey's honest significant difference (HSD) test $(p<0.01)$; $p$-values for all pairwise comparisons are presented in Supplementary file 11.

The online version of this article includes the following source data and figure supplement(s) for figure 5:

Source data 1. Raw data of Pikp-ancHMA $R_{\max } S P R$.

Figure supplement 1. Co-immunoprecipitation experiment between AVR-PikD and the two plausible historical states of the IAOVV/LVKIE region within Pikp-HMA.

Figure supplement 2. Replicates of the co-immunoprecipitation (co-IP) experiments between the Pikp-1:ancHMA IAQVV/LVKIE mutants and AVR-PikD.

Figure supplement 3. Purified proteins used in surface plasmon resonance studies.

Figure supplement 4. Surface plasmon resonance (SPR) results show the effect of the IAQVV-LVKIE mutations on the AVR-PikD binding, as indicated by \% $R_{\max }$.

Figure supplement 5. The AV-VE (Ala-222-Val and Val-230-Glu) substitutions are sufficient to increase binding affinity towards the AVR-PikD effector in co-immunoprecipitation (co-IP).

\section{High binding affinity to AVR-PikD accounts for the capacity of Pikp-1: ancHMA to trigger an immune response}

To test if effector binding by Pikp-1:ancHMA is sufficient to trigger an immune response, we performed HR cell death assays by transiently co-expressing each of the Pikp-1:ancHMA fusions with AVR-PikD and Pikp-2 in Nicotiana benthamiana. We discovered that all Pikp-1:ancHMA variants are autoactive and trigger spontaneous cell death in the absence of the effector (Figure 6-figure supplement 1, Figure 6-figure supplement 2). Notably, the presence of the Pikp-2 partner is required for Pikp-1:ancHMA autoactivity.

Next, we used previously generated ancHMA chimeras to delimitate the region responsible for the autoactivity phenotype of Pikp-1:ancHMA. We tested these fusions for loss of function in cell death assays by transient co-expression with Pikp-2 in N. benthamiana (Figure 6-figure supple-

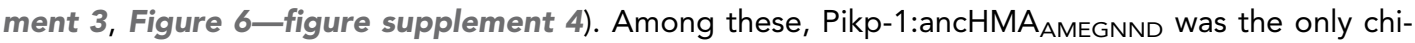
mera to show complete loss of autoactivity. This phenotype was not due to protein instability or low protein abundance (Figure 4C, Figure 4-figure supplement 1). These results suggest that the PMASDKH/AMEGNND region, located in the $\beta 1-\alpha 1$ and $\alpha 2-\beta 4$ loops of the Pikp-HMA domain, underpins Pikp-1:ancHMA autoactivity.

To determine whether gain of AVR-PikD binding results in a functional immune response, we performed cell death assays using Pikp-1:ancHMA mutants in the IAQVV/LVKIE region. We first removed autoactivity by introducing AMEGNND mutations into these constructs (Figure 6A), henceforth called Pikp-1:ancHMA ${ }_{L V K I E}{ }^{*}$, Pikp-1:ancHMA ${ }_{L A K I E}{ }^{*}$, Pikp-1:ancHMA ${ }_{\text {LAKIV }}{ }^{*}$, Pikp-1:ancHMALAKVV ${ }^{\star}$, Pikp-1:ancHMA LAOVV $^{\star}$. None of the resulting mutants triggered spontaneous cell death when transiently co-expressed with Pikp-2 (Figure 6B, C Figure 6-figure supplement 5). Co-expression with AVR-PikD revealed that the strength of binding directly correlates with the strength of HR. The mutants that gained AVR-PikD binding in the co-IP and SPR experiments, namely Pikp-1:ancHMALAKIE $^{*}$ and Pikp-1:ancHMA ${ }_{L V K I E}{ }^{*}$, showed HR phenotypes. The Pikp-1:ancHMA ${ }_{\text {LVKIE }}{ }^{*}$ mutants triggered cell death at levels similar to Pikp-1, whereas the HR triggered by Pikp-1:ancHMA $A_{L A K I E}{ }^{*}$ was slightly, yet significantly, reduced when compared to Pikp-1. By contrast, Pikp-1:ancHMA*, Pikp-1: anCHMA LAKVV $^{*}$, and Pikp-1:anCHMA $A_{L A O V V^{*}}$ did not elicit cell death above background levels. All proteins accumulated at similar levels in western blot analysis (Figure 6-figure supplement 6). Overall, these results indicate that the adaptive mutations in the IAQVV/LVKIE region towards AVR-PikD binding at high affinity also enable effector-dependent activation of the cell death immune response. 


\section{A}

B
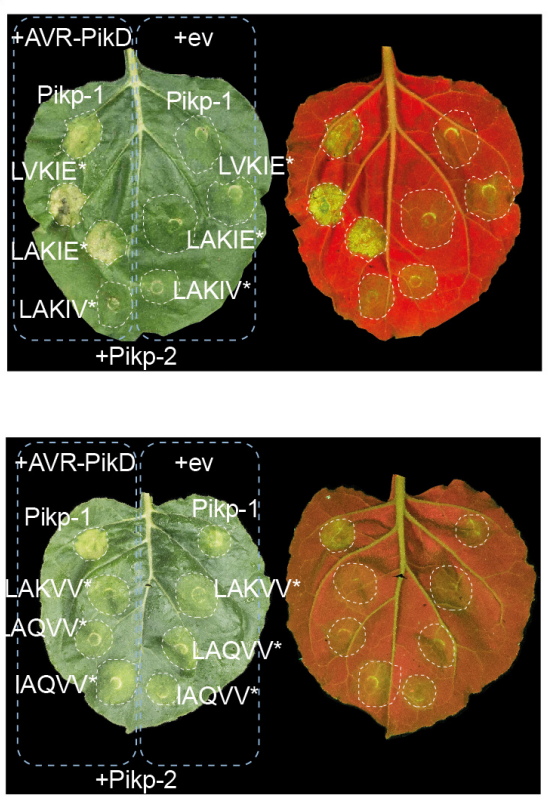

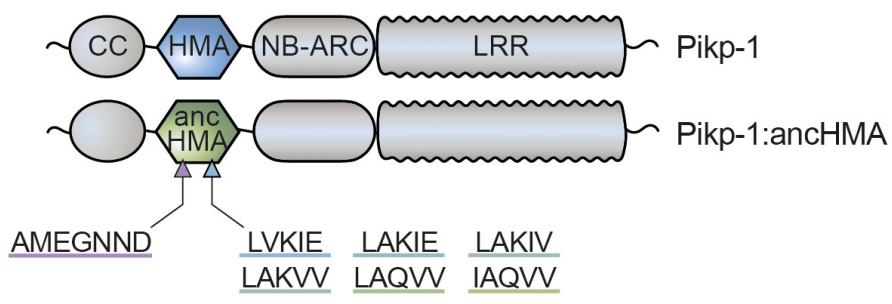

C
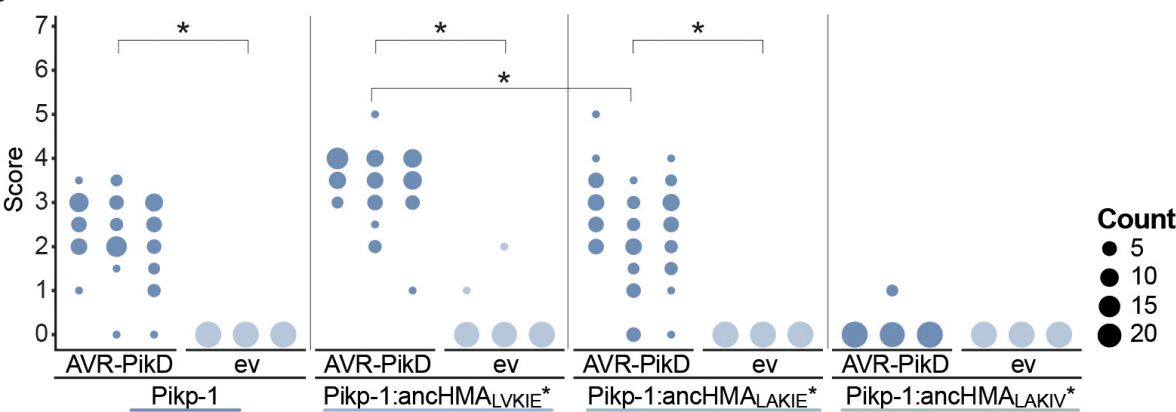

Pikp-1:ancHMALAKIE* ${ }^{*}$ Pikp-1:ancHMALAKIV*
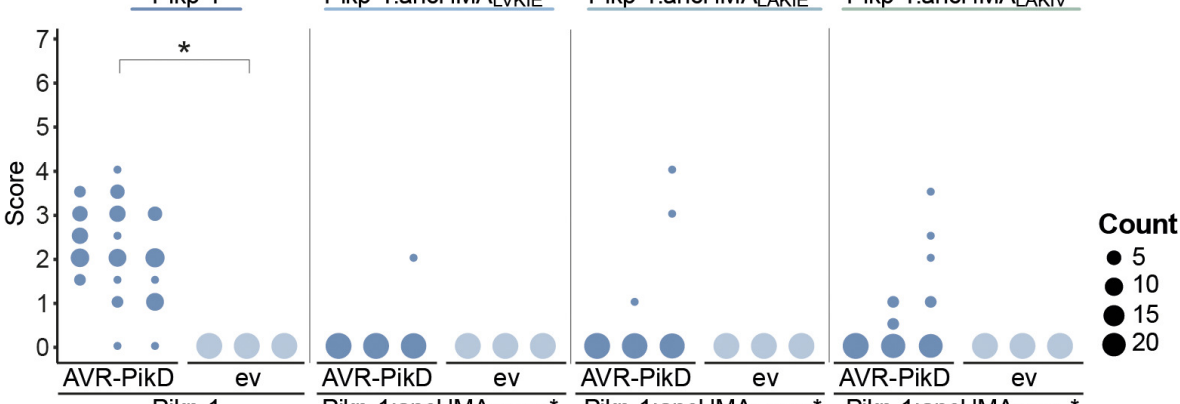

Figure 6. Pikp-1:ancHMA $A_{\text {LVKIE }}{ }^{*}$ and Pikp-1:ancHMA ${ }_{\text {LAKIE }}{ }^{*}$ mediate immune response towards the AVR-PikD effector. (A) Schematic representation of wild-type Pikp-1 and Pikp-1:ancHMA fusions used in the assay. The mutated regions are presented with arrowheads and listed. (B) Representative images of hypersensitive response (HR) cell death assay after transient co-expression of the Pikp-1:ancHMA* mutants (C-terminally tagged with HF) with AVR-PikD ( $\mathrm{N}$-terminally tagged with Myc) and Pikp-2 (C-terminally tagged with HA). Empty vector (ev) was used as a negative control. All constructs were co-expressed with the gene silencing suppressor p19 (Win and Kamoun, 2003). The leaves were photographed 5 days after infiltration under daylight (left) and UV light (right). (C) HR was scored at 5 days post-agroinfiltration. The results are presented as dot plots, where the size of a dot is proportional to the number of samples with the same score (count) within the same biological replicate. The experiment was independently repeated at least three times with 23-24 internal replicates; the columns within tested conditions (labelled on the bottom) correspond to results from different biological replicates. Significant differences between relevant conditions are marked with an asterisk (*); details of the statistical analysis are summarised in Figure 6-figure supplement 5.

The online version of this article includes the following source data and figure supplement(s) for figure 6:

Source data 1. Hypersensitive response scores for IAQV to LVKIE mutations in Pikp-HMA.

Figure supplement 1. Pikp-1:ancHMA fusions are autoactive in a Pikp-2-dependent manner.

Figure supplement 1-source data 1. Hypersensitive response scores used in Figure 6-figure supplement 1.

Figure supplement 2. Statistical analysis of hypersensitive response cell death for the Pikp-1:ancHMA fusions.

Figure supplement 3. The AMEGNND mutations within ancestral HMA (ancHMA) abolish autoactivity.

Figure supplement 3-source data 1. Hypersensitive response scores used in Figure6-figure supplement 3.

Figure supplement 4. Statistical analysis of cell death assay for the Pikp-1:ancHMA chimeras.

Figure supplement 5. Statistical analysis of cell death for the Pikp-1:ancHMA mutants within the IAOWV/LVKIE region.

Figure supplement 6. In planta accumulation of the Pikp-1:ancHMA* mutants in the IAQVV/LVKIE region. 


\section{A distinct region (MKANK/EMVKE) in the integrated HMA domain of Pikm-1 determines high-affinity AVR-PikD binding}

As noted above, the LVKIE polymorphisms are relatively rare among Pik-1 allelic variants and Oryza orthologues (2 out of 19 examined sequences) (Figure 7-figure supplement 1). Other rice allelic variants of Pik-1 retain the predicted IAOVV ancestral state. Interestingly, Pikm-1, a Pik-1 allelic variant with the IAOVV residues, binds the AVR-PikD effector with high affinity and triggers an immune response upon effector recognition (De la Concepcion et al., 2018; Kanzaki et alo, 2012). This led us to hypothesise that the integrated HMA domain of Pikm-1 (Pikm-HMA) has undergone a distinct evolutionary path towards AVR-PikD binding compared to Pikp-HMA.

To determine which Pikm-HMA mutations have enabled gain of AVR-PikD binding, we performed structure-informed sequence comparison of the Pikm-HMA and ancHMA domains similar to the approach described above for Pikp-1. We amended the sequence of previously predicted ancHMA with a three-amino-acid-long extension (residues 262-264 of the full-length Pikm-1) that includes residues that are polymorphic in Pikm-HMA but identical between ancHMA and Pikp-HMA. Next, we mapped five polymorphic regions that differentiate the ancHMA from modern Pikm-HMA (Figure 7A, B), introduced mutations in these regions in Pikm-1:ancHMA, and subjected the Pikm-1: ancHMA variants to in planta co-IP with AVR-PikD. Among the five chimeras tested in this experiment, Pikm-1:ancHMA $A_{E M V K E}$ was the only one to associate with AVR-PikD (Figure 7C, Figure 7-figure supplement 2). Among the remaining chimeras, Pikm-1:ancHMA $A_{V H}$ protein was unstable and hence yielded inconclusive results. Overall, we conclude that Pikm-HMA evolved towards association with AVR-PikD through mutations in the MKANK/EMVKE region, a distinct interface from the IAQVV/LVKIE region of Pikp-1.

\section{The ANK-VKE mutations confer high-affinity AVR-PikD binding in Pikm- HMA}

We reconstructed the mutational history of the MKANK/EMVKE interface to trace the evolutionary trajectory of Pikm-HMA detection of AVR-PikD (Figure 8A). The ASR was performed by a combination of manual and probability-based approaches using a protein sequence alignment and a representative phylogenetic tree of the HMA domain, where ancHMA and Pikm-HMA were separated by four internal nodes (Figure 3-figure supplement 2). However, we could only identify one node that represents an evolutionary intermediate between the ancestral MKANK and present-day EMVKE states, namely EMANK, that emerged through MK-EM mutations (M188E and K189M). The ANKVKE mutations (A261V, N262K, and K263E) were acquired at a later timepoint, and determining the order of individual mutations was not possible given the limits of the phylogenetic tree resolution.

To evaluate the impact of these historical mutations, we generated the ancHMA ${ }_{E M A N K}$ mutant that recapitulates the predicted step-by-step intermediate state of the MKANK/EMVKE region, incorporated this mutant into the Pikm-1 backbone, and assayed it for in planta association with AVR-PikD. By contrast to Pikm:ancHMA $A_{E M V K E}$, Pikm:ancHMA $A_{\text {EMANK }}$ did not gain the capacity to associate with AVR-PikD relative to Pikm:ancHMA ${ }_{\text {MKANK }}$ (Figure $8 B$, Figure 8-figure supplement 1).

Next, we validated these results in vitro using the AVR-PikD protein and the full set of ancHMA mutants purified from E. coli (Figure 8-figure supplement 2). To encompass the full diversity between the ancestral and present-day states of Pikm-HMA, we used HMA sequences with a fiveamino acid extension at the $\mathrm{C}$-terminus (ancHMA+5) compared to the constructs used in the PikpHMA experiments. During protein purification, we noted a shift in elution volume of the ancHMA+5

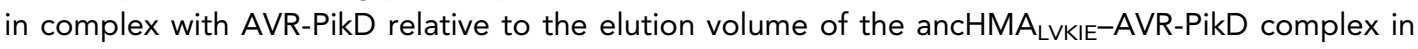
size-exclusion chromatography (Figure 8-figure supplement 3). We concluded that this shift is consistent with different stoichiometries of the ancHMA-AVR-PikD complexes; while anCHMA LVKIE- $^{-}$ AVR-PikD formed a two-to-one complex, the constructs with the extension interacted with the effector at a one-to-one ratio. Accounting for this stoichiometry, we carried out SPR experiments using the same experimental design as in the Pikp-HMA assays and discovered that among tested mutants the ancHMA $A_{E M V K E}$ displayed the highest rates of interaction with AVR-PikD, followed by ancHMAE-

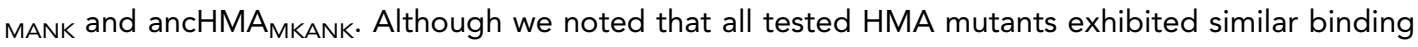
affinity to AVR-PikD at $400 \mathrm{nM}$ concentration (Figure 8-figure supplement 4; Supplementary file $1 L$ ), they displayed marked differences in the shapes of their sensorgrams (Figure 8C, D, Figure 8figure supplement 4, Figure 8-figure supplement 5). First, despite high values for 'binding', 
A

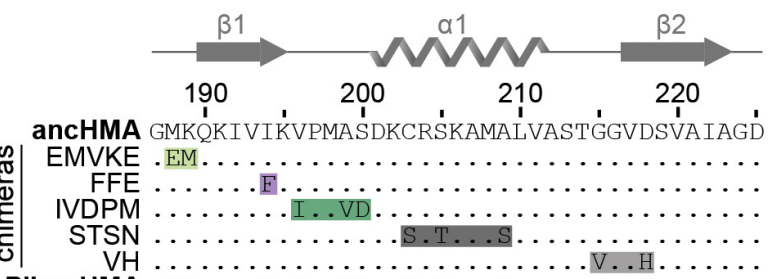

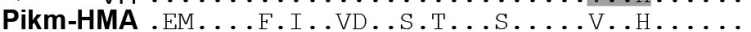

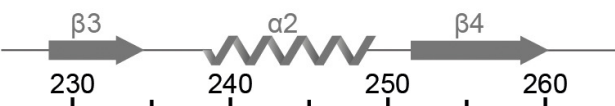

ancHMA LRDQVVVVGDGIDS IKLVSALRKKVGHAELLQVSQANKD

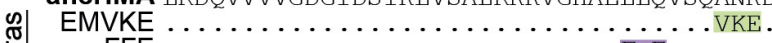

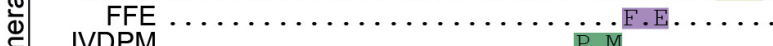

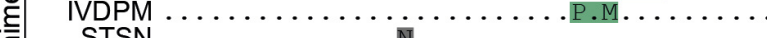

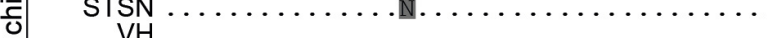

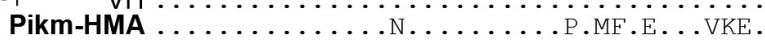

B

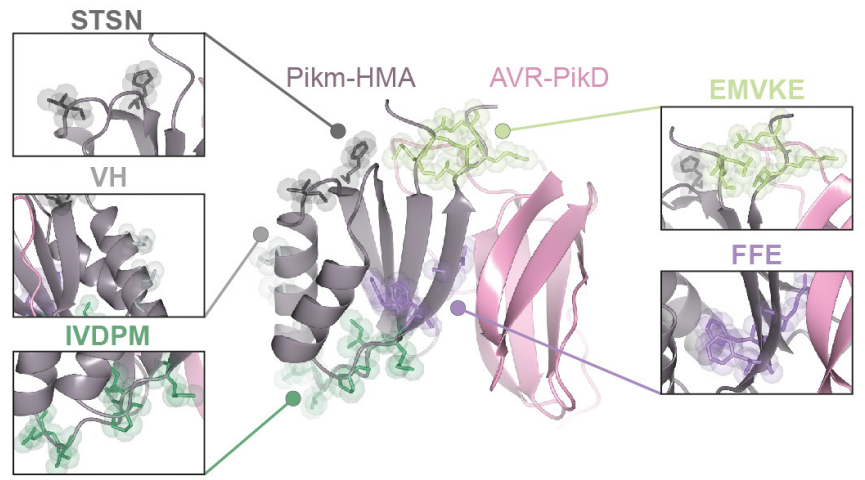

C

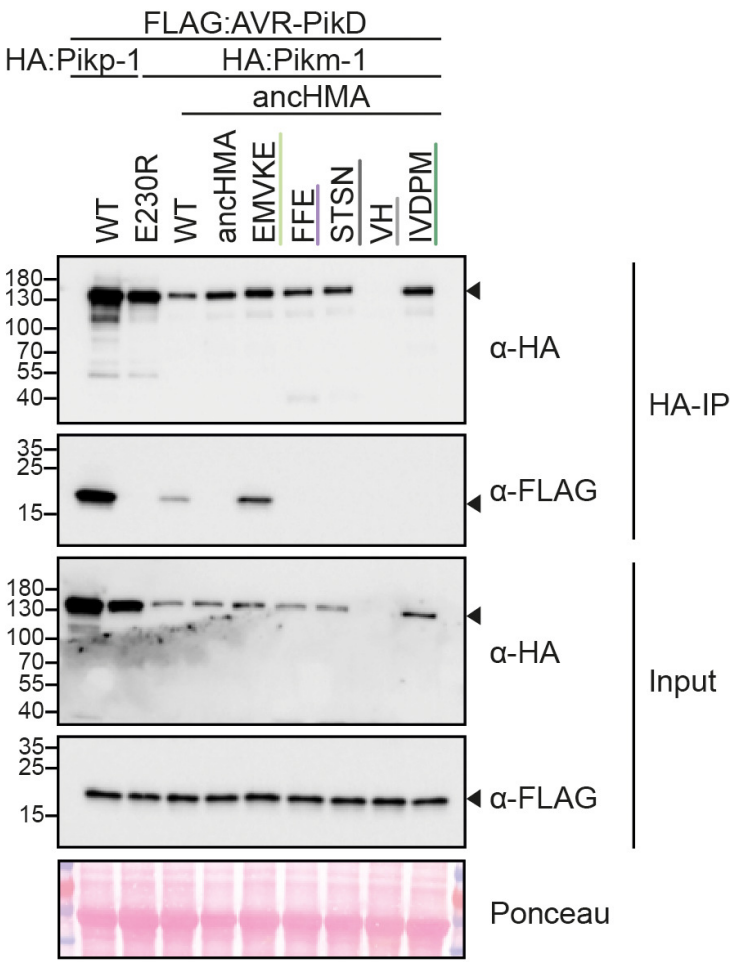

Figure 7. The MKANK/EMVKE region of the heavy metal-associated (HMA) domain of Pikm-1 determines high-affinity AVR-PikD binding. (A) Protein sequence alignment between the ancestral HMA (ancHMA), Pikm-HMA, and Pikm-ancHMA chimeras. The protein model above the alignment depicts Pikm-HMA secondary structure. The colour-coded rectangles mark polymorphic regions used for chimeric swaps. (B) Schematic representation of the Pikm-HMA domain (purple) in complex with AVR-PikD (pink) (De la Concepcion et al., 2018), with polymorphic regions between Pikm-HMA and ancHMA colour-coded as in (A). The molecular surfaces of the polymorphic residues are also shown. (C) EMVKE substitutions in the ancHMA restore in planta association with AVR-PikD. Co-immunoprecipitation experiment between AVR-PikD (N-terminally tagged with FLAG) and Pikp-1:ancHMA chimeras (N-terminally tagged with FLAG), labelled above. Wild-type (WT) Pikp-1/Pikm-1 and Pikp-1E230R were used as positive and negative controls, respectively. Immunoprecipitates (HA-IP) obtained with anti-HA probe and total protein extracts (input) were immunoblotted with the appropriate antisera (labelled on the right). Rubisco loading control was carried out using Ponceau staining. Arrowheads indicate expected band sizes. Three independent replicates of this experiment are shown in Figure 7-figure supplement 2. The online version of this article includes the following figure supplement(s) for figure 7 :

Figure supplement 1. Protein sequence alignment of the heavy metal-associated (HMA) domain from the Oryza spp.

Figure supplement 2. Replicates of the co-immunoprecipitation (co-IP) experiment between the Pikm-1:ancHMA chimeras and AVR-PikD.

ancHMA exhibited high off-rates, as illustrated by the pattern of 'dissociation' and shape of the curves. Second, ancHMA $A_{E M V K E}$ displayed high values for 'binding' and 'dissociation', with low $\Delta \%$ $\mathrm{R}_{\max }$ indicating tight and stable binding. Finally, ancHMA $\mathrm{EMANK}_{\mathrm{f}}$ fell in-between ancHMA and ancHMA $A_{E M V K E}$, with stable and relatively low $\Delta \% R_{\max }$ at the top concentration and moderate $\Delta \%$ $\mathrm{R}_{\max }$ at lower concentrations. These findings indicate that the ANK-VKE substitutions are essential for Pikm-HMA high-affinity binding of AVR-PikD. Altogether, both co-IP and SPR experiments indicate that the MKANK/EMVKE region plays an important role in high-affinity binding of the AVR-PikD effector by Pikm-HMA.

We further noted that the ANK-VKE substitutions are present in three Pik-1 alleles of rice, namely closely related Pik*-1 (Zhai et al., 2011), Pikm-1 (Ashikawa et al., 2008), and Piks-1 (Jia et al., 2009; Figure 7-figure supplement 1). Pikm-1 differs from Piks-1 and Pik*-1 by only two and eight 
A

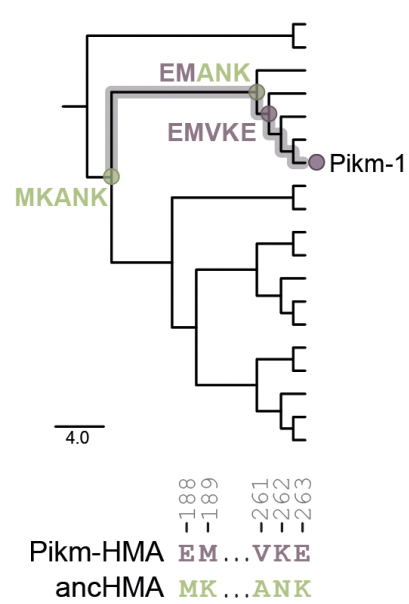

B

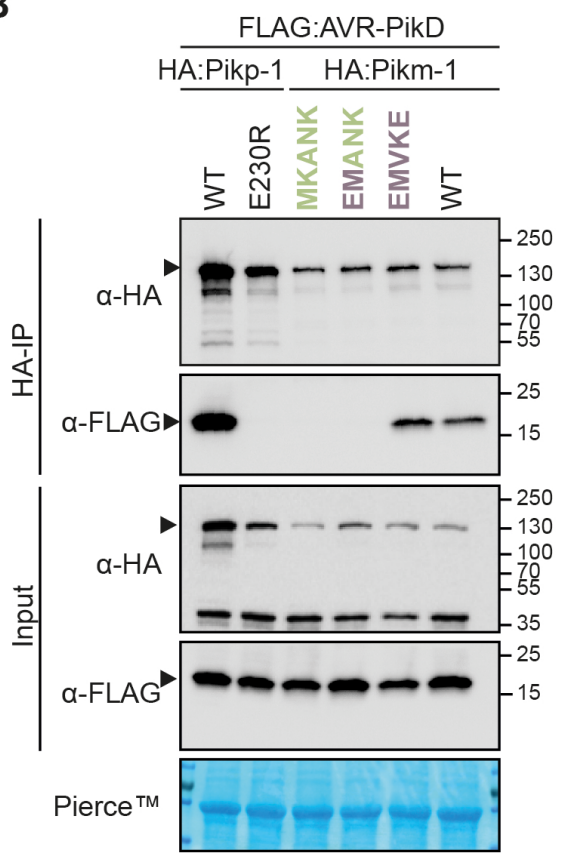

C

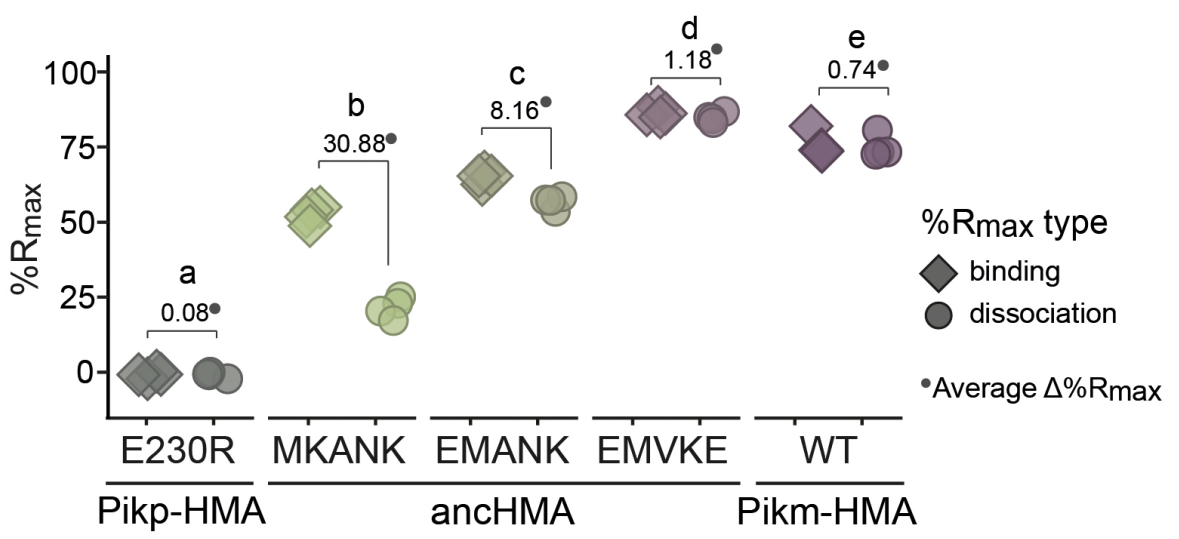

D
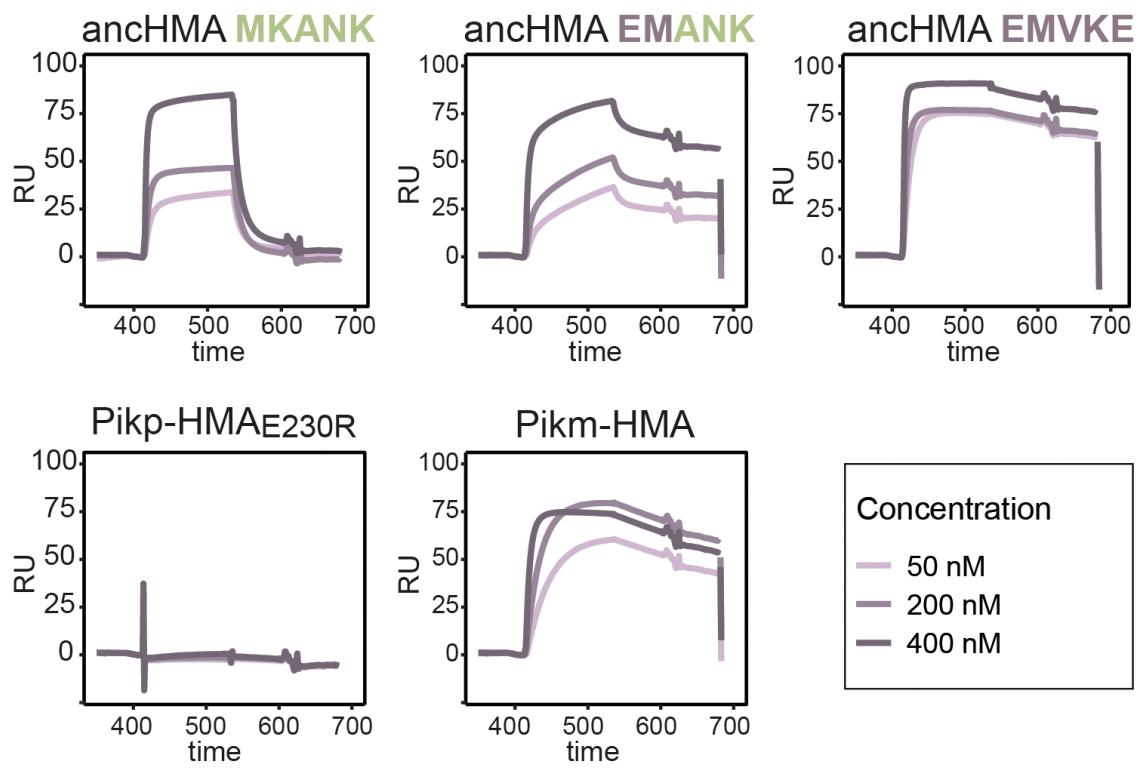

Figure 8. The ANK-VKE substitutions are essential for Pikm-HMA adaptation towards high-affinity binding to AVR-PikD. (A) Schematic representation of the neighbour joining (NJ) tree of the ancestral HMA (HMA) domains from Oryza spp. (shown in Figure 3-figure supplement 2). The scale bar indicates the evolutionary distance based on the number of base substitutions per site. Historical substitutions in the MKANK/EMVKE region acquired over the course of Pikm-HMA evolution are shown next to the corresponding nodes. The mutations are colour-coded to match the ancestral (green) and present-day (purple) states. (B) Co-immunoprecipitation experiment illustrating in planta association of AVR-PikD (N-terminally tagged with FLAG)

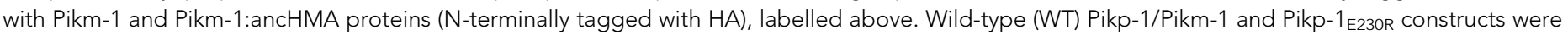
used as positive and negative controls, respectively. Immunoprecipitates (HA-IP) obtained using anti-HA probes and total protein extracts (input) were immunoblotted with the appropriate antisera (depicted on the left). The arrowheads indicate expected band sizes. Rubisco loading control was performed using Pierce solution. Three independent replicates of this experiment are shown in Figure 8-figure supplement 1. (C) Plot illustrating calculated percentage of the theoretical maximum response $\left(\% \mathrm{R}_{\max }\right)$ values for interaction of heavy metal-associated (HMA) analytes, labelled below, with AVR-PikD ligand (C-terminally tagged with HIS) determined by surface plasmon resonance (SPR). \% $\mathrm{R}_{\max }$ was calculated assuming a one-to-one (HMA-to-effector) binding model for Pikm-HMA and ancHMAs, and a two-to-one for Pikp-1 E230R. The values were normalised for the amount of ligand immobilised on the NTA-chip. The chart summarises the results obtained for HMA analytes at $200 \mathrm{nM}$ concentration from five independent experiments, with all the data points represented as diamonds ('binding') or circles ('dissociation'). Three different concentrations of analytes (400 $\mathrm{nM}$, $200 \mathrm{nM}, 50 \mathrm{nM}$ ) were tested; results for $400 \mathrm{nM}$ and $50 \mathrm{nM}$ concentrations are shown in Figure 8-figure supplement 4 . Average $\Delta \% \mathrm{R}_{\max }(\bullet)$ values represent absolute differences between values for 'binding' and 'dissociation', calculated from the average values for each sample, and serve as an offFigure 8 continued on next page 
Figure 8 continued

rate approximate. Statistical differences among the samples were analysed with Tukey's honest significant difference (HSD) test ( $p<0.01)$; $p$-values for all pairwise comparisons are presented in Supplementary file $1 \mathrm{~K}$. (D) The SPR sensorgrams of the AVR-PikD and HMA proteins, corresponding to the data used in (C). Independent replicates of this experiment are presented in Figure 8-figure supplement $\mathbf{5}$.

The online version of this article includes the following source data and figure supplement(s) for figure 8:

Source data 1. Raw data of Pikm-ancHMA $R_{\max }$ SPR.

Figure supplement 1. Replicates of the co-immunoprecipitation experiment between Pikm-1:ancHMA mutants in the MKANK/EMVKE region and AVRPikD.

Figure supplement 2. Purified proteins used in surface plasmon resonance studies.

Figure supplement 3. Different stoichiometry of the ancHMA-AVR-PikD complexes.

Figure supplement 4. Surface plasmon resonance (SPR) results showing the effect of the step-by-step mutations within the MKANK/EMVKE region on the AVR-PikD binding in vitro, as indicated by \% $R_{\max }$.

Figure supplement 5. The surface plasmon resonance (SPR) sensorgrams for the AVR-PikD-HMA binding.

amino acid polymorphisms, respectively, but no synonymous changes (Supplementary file 1J). This demonstrates a very recent emergence of these Pik-1 alleles and their associated ANK-VKE substitutions. Next, we aimed to determine whether the gain of AVR-PikD binding translates to an immunoactive Pikm-1:ancHMA by means of HR cell death assay. However, while addressing this question we run into several technical problems, including (1) autoactivity of Pikm-1:ancHMA, (2) perturbed response to AVR-PikD, (3) reduced protein accumulation levels, and (4) weak/inconsistent HR (Bialas et al., 2021). These precluded reliable studying of how the strength of AVR-PikD binding correlates with $\mathrm{HR}$ cell death.

\section{Pikp-1 and Pikm-1 NLR receptors convergently evolved through distinct biochemical paths to gain high-affinity AVR-PikD binding}

Our findings led us to develop an evolutionary model that depicts convergent molecular evolution of Pikp-1 and Pikm-1 towards AVR-PikD binding (Figure 9). To interpret this model from a structural perspective, we attempted to determine crystal structures of the ancHMA domains in complexes with AVR-PikD. Crystallisation screens of the heterologously expressed proteins resulted in crystals of the ancHMA $A_{L V K I E}-A V R-P i k D$ complex, which diffracted to $1.32 \AA$ resolution (Supplementary file $1 L$ ). The structure revealed an overall architecture of the complex similar to that of previously published co-structures of Pik-HMAs and AVR-PikD (Figure 9-figure supplement 1A; De la Concepcion et al., 2018; De la Concepcion et al., 2021; Maqbool et al., 2015). We note that the MKANK/ EMVKE and IAQVV/LVKIE regions map to two of the three interaction interfaces previously described to underpin binding of AVR-PikD, and other AVR-Pik variants, to Pik-HMAs (De la Concepcion et al., 2021; De la Concepcion et al., 2019; De la Concepcion et al., 2018).

To gain insights into the structural determinants of effector binding in the IAOVV/LVKIE region, we generated a homology model of the ancHMA in complex with AVR-PikD (Figure 9-figure supplement 1B). We further validated modelled interactions by examining the published structure of Pikm-HMA (De la Concepcion et al., 2018), whose IAQVV/LVKIE region is identical to ancHMA. Close inspection of these structures revealed that the Val-230-Glu (V230E) substitution enhances the interaction with AVR-PikD through hydrogen bond formation with His-46 (Figure 9A, Figure 9-figure supplement $1 \mathrm{C}$ ). This bond is formed by Glu-230 (E-230) of ancHMA HMA and ancHMA, which carry Val-230 (V-230) at the structurally equivalent position.

Next, we examined the structural basis of the interaction of the MKANK/EMVKE region with AVR-PikD by comparing Pikm- and Pikp-HMA structures (De la Concepcion et al., 2018) that feature EMVKE and LKANK residues (reminiscent of the MKANK amino acids present in ancHMA), respectively. In both cases, Lys-262 (K262) is a major effector-binding determinant that forms hydrogen bonds or salt bridges with Glu-53 and Ser-72 of AVR-PikD (Figure 9A). However, in Pikm-HMA the position of Lys-262 (K262) is structurally shifted causing a difference in the conformation of the HMA peptide backbone, and associated side chains, compared to Pikp-HMA. Homology modelling fails to predict this change in the HMA backbone that results in tighter interaction between AVR-PikD and Pikm-HMA compared to Pikp-HMA (De la Concepcion et al., 2021; De la Concepcion et al., 2019; De la Concepcion et al., 2018). We conclude that Asn-262-Lys (N262K) and Lys-263-Glu (K263E) of 
A

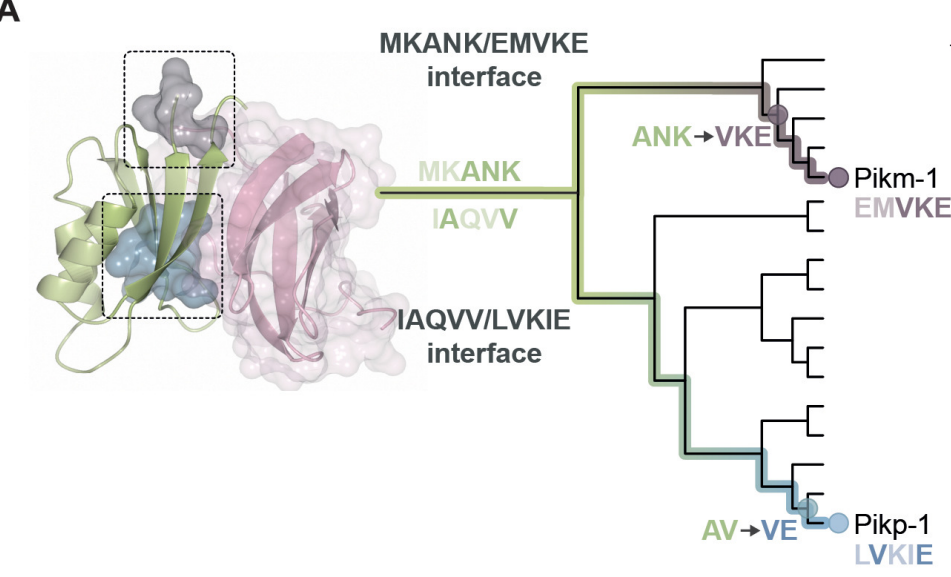

MKANK/EMVKE interface
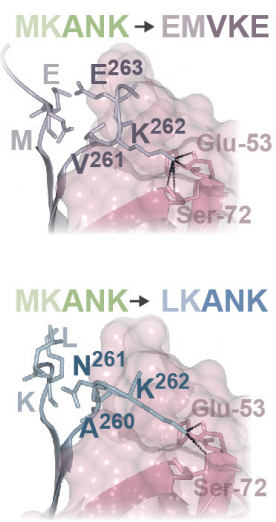

IAQVVILVKIE interface
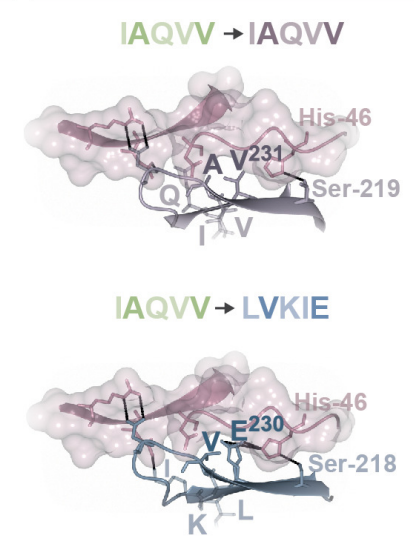

B

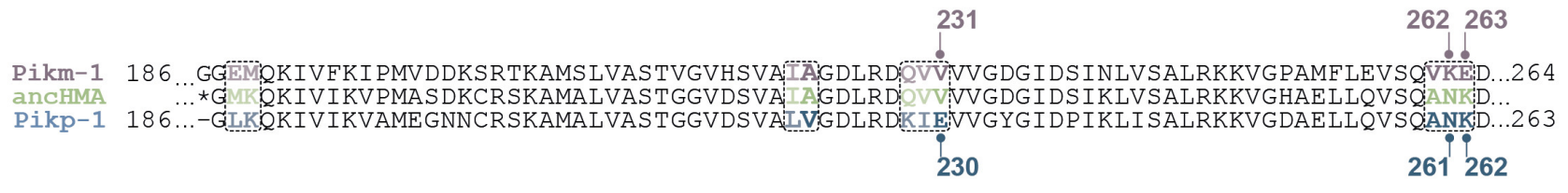

C

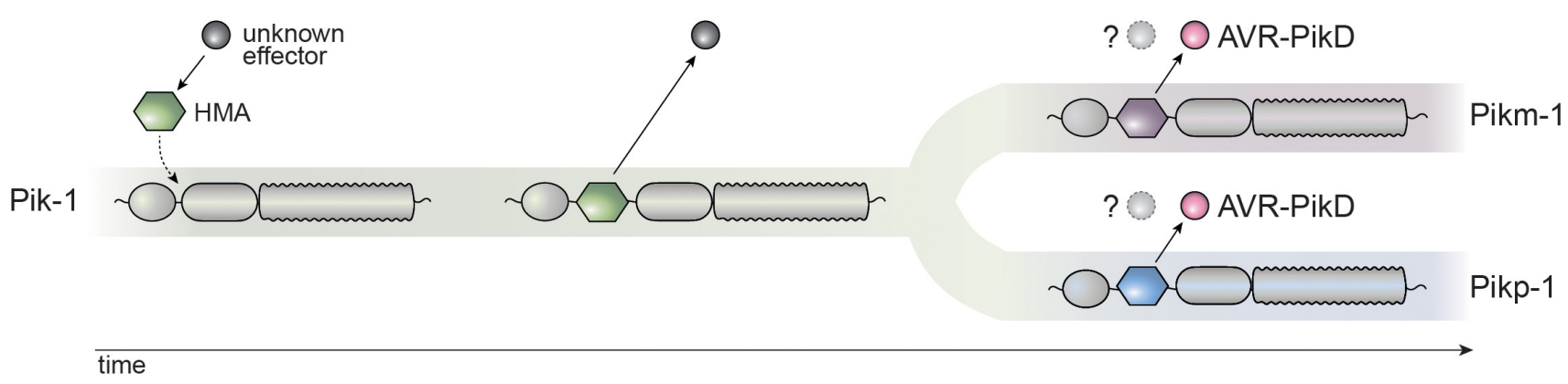

Figure 9. Model of molecular convergence of Pikp-1 and Pikm-1 towards AVR-PikD binding at high affinity. (A) The heavy metal-associated (HMA) domains of Pikp-1 and Pikm-1 receptors have convergently evolved through distinct evolutionary and biochemical paths to bind AVR-PikD with high affinity. The Pikp-HMA domain evolved through the AV-VE adaptations in the IAQVV/LVKIE region, whereas Pikm-HMA domain acquired the ANK-VKE mutations in the MKANK/EMVKE region. Schematic representations of the HMA-AVR-PikD structures, adapted from De la Concepcion et al., 2018, are presented with selected side chains shown as sticks and labelled; the colours of the residue labels match colours of the respective molecules. Dashed lines stand for hydrogen bonds or salt bridges. (B) The protein sequence alignment between Pikp-HMA, Pikm-HMA, and ancestral HMA (ancHMA), with relevant amino acids marked. (C) We propose a model in which the HMA effector target integrated into Pik-1 to bait the recognition of an unknown effector. Throughout evolution the Pik-1 receptor and its integrated HMA domain diversified and led to the emergence of the Pikp-1 and Pikm-1 allelic variants that bind newly emerged AVR-PikD effector.

The online version of this article includes the following figure supplement(s) for figure 9:

Figure supplement 1. The Val-230-Glu mutation within the LVKIE region of ancestral HMA (ancHMA) enhances interaction with AVR-PikD through hydrogen bond formation.

the ANK-VKE substitution likely determine differential binding between the ancestral and presentday Pikm-HMA domains.

\section{Discussion}

The molecular evolution events associated with the transition of NLR IDs from pathogen effector targets to baits remain elusive. Here, we investigated the evolution of these unconventional domains of NLR receptors using rice Pik as a model system. First, we performed extensive phylogenetic analyses 
to determine that the integration of the HMA domain emerged over 15 MYA, predating the radiation of Oryzinae (Figure 1D). Using sequence reconstruction and resurrection of an ancestral integrated HMA domain that dates back to early divergence of Oryza spp., we showed that the capacity of Pik-1 to sense and respond to AVR-PikD evolved relatively recently through distinct evolutionary and biochemical paths in two alleles of Pik-1, Pikp-1 and Pikm-1. This combination of evolutionary and biochemical approaches allowed us to develop a model of the adaptive evolution of the Pik proteins towards high-affinity AVR-PikD binding (Figure 9).

The molecular bases of functional transitions in NLR evolution remain poorly understood, especially over extended timescales. Here, we showed that adaptive evolution of Pikp-1 and Pikm-1 from weak to high-affinity binding to the AVR-PikD effector involves two distinct regions within the HMA domain. Overall, these interfaces seem to function in a synergistic yet interchangeable manner, such that weak interaction at one interface can be compensated by strong interaction at a different one (De la Concepcion et al., 2021; De la Concepcion et al., 2018). We propose that this modularity between different regions of the HMA increases the HMA's capacity for rapid adaptive evolution as it can follow alternative mutational paths to produce similar phenotypic outcomes and counteract rapidly evolving pathogen effectors. Indeed, HMA domains can also detect another $M$. oryzae effector AVR-Pia through an alternative interface (Guo et al., 2018; Varden et al., 2019), further illustrating the capacity of the HMA domain to bait pathogen effectors through different interfaces. This may have contributed to the recurrent emergence of HMAs as NLR IDs. Previous studies have revealed that HMAs have independently integrated into NLR immune receptors from at least four flowering plant families (Kroj et al., 2016; Sarris et al., 2016).

The HMA domain of Pik-1 exhibits signatures of positive selection in contrast to the NB-ARC domain (Figure 2), likely reflecting coevolution with pathogen effectors versus overall purifying selection. This further suggests that HMA domains are malleable platforms that can accommodate accelerated mutational rates (Białas et al., 2018; Costanzo and Jia, 2010). Similar observations have previously been made in a number of plant NLRs, whose individual domains display patterns of asymmetrical evolution and distinct rates of selection, suggesting that NLRs evolve in a modular fashion (Kuang et al., 2004; Maekawa et al., 2019; Prigozhin and Krasileva, 2020; Read et al., 2020; Seeholzer et al., 2010). Moreover, having a domain responsible for effector recognition may release other domains from the pressure of diversification and reduce the risk of compromising or mis-regulating NLR activity (Cesari, 2018). In addition, coupling with a helper NLR such as Pik-2 likely provides yet another mechanism of functional compartmentalisation, further enhancing the evolvability of the sensor by freeing it from the constraint of executing the hypersensitive cell death (Adachi et al., 2019; Cesari, 2018; Wu et al., 2018).

We showed that the evolutionarily derived AV-VE in Pikp-1 (Figure 5) and ANK-VKE polymorphisms in Pikm-1 (Figure 8) enabled high-affinity binding to AVR-PikD. Although the high sequence divergence and elevated mutation rates among HMA sequences precluded rigorous dating of the emergence of these key adaptations, the low level of total nucleotide polymorphisms among closely related Pik alleles-in particular, the very few synonymous substitutions among Pikp- and Pikmrelated alleles-points to a very recent emergence of the adaptive polymorphisms. Given that the rice-infecting lineage of $M$. oryzae is estimated to have arisen about 7000-9000 years ago (Couch et al., 2005; Latorre et al., 2020), our findings are consistent with the view that Pik-1 alleles evolved during rice domestication as previously suggested (Kanzaki et al., 2012; Zhai et al., 2011). In addition, AVR-Pik is widespread in rice-infecting isolates but absent in other blast fungus lineages (Bentham et al., 2021; Langner et al., 2021; Latorre et al., 2020; Yoshida et al., 2016). Therefore, it is tempting to speculate that the rice agroecosystem has created the ecological context that led to Pik neofunctionalisation towards recognition of the new pathogen threat imposed by the blast fungus. Different rice populations may have independently encountered fungal pathogens carrying AVR-Pik, leading to intense natural selection and independent emergence of the Pikp and Pikm adaptations.

We concluded that the Pik-1-integrated HMA domain did not function in sensing AVR-PikD for most of its over 15-million-year-long evolutionary history, inviting the question about the role of the ancestral integrated HMA. It is likely that over millions of years, prior to rice domestication, the Pik-1 HMA domain had recognised effectors other than AVR-Pik. These could be other members of AVRPik-like (APikL) effector family (Bentham et al., 2021) or their ancestors, the structurally related MAX-effectors-an ancient effector family present across blast lineages and other fungal pathogens 
(de Guillen et al., 2015; Petit-Houdenot et al., 2020)_or effectors from other plant pathogen taxa. Indeed, the HMA domain is known to bind effectors from diverse pathogens including bacteria and oomycetes, in addition to fungi (González-Fuente et al., 2020). Karasov et al., 2014 proposed that NLRs caught in pairwise arms races (one NLR recognising one effector) are likely to be shortlived, whereas NLRs entangled in diffuse evolution (functioning against multiple effectors and/or multiple pathogens) are more likely to persist over longer timescales. Our model paints a more complex picture of the macroevolutionary dynamics of NLR-IDs. These receptors have the capacity to switch from one effector to another, while also engaging in short-term arms race dynamics, as seems to be the case of Pik-1 vs. AVR-Pik (Bialas et al., 2018; Kanzaki et al., 2012). It is remarkable that the Pik-1 gene and its paired Pik-2 gene have been maintained in grass populations for tens of millions of years, even after the integration of the HMA domain. This points to a successful evolutionary strategy for generating long-lived disease resistance traits, with HMA promiscuity towards pathogen effectors at the centre of this model.

We discovered that the Pikp-1:ancHMA fusions trigger spontaneous hypersensitive cell death when co-expressed with Pikp-2 and mapped the region responsible for the autoactivity to two HMA parallel loops, $\beta 1-\alpha 1$ and $\alpha 2-\beta 4$ (Figure 6-figure supplement 1, Figure 6-figure supplement 3). Although the precise mechanism underpinning this autoactivity remains to be elucidated, we propose that coevolution of the HMA with the canonical domains of Pik-1 and/or Pik-2 drives this molecular incompatibility. Mismatching domains from different evolutionary timepoints may disrupt fine-tuned biochemical interactions between HMA and other domains. Indeed, intra- and intermolecular incompatibilities of NLRs are known causes of autoimmunity in plants (Harris et al., 2013; Li et al., 2020b; Lukasik-Shreepaathy et al., 2012; Qi et al., 2012; Rairdan and Moffett, 2006; Tran et al., 2017; Wang et al., 2015). We further noted that some Pik-1 orthologues, namely LpPik1 and N-type Pik-1 genes, carry large deletions within their HMAs, which may have emerged to eliminate autoimmunity (Figure 2-figure supplement 1). This is consistent with the view that the risk of autoactivity acts as a strong evolutionary constraint narrowing NLR mutational pathways (Chae et al., 2014).

We uncovered a rich genetic diversity of Pik genes beyond Oryza species (Mizuno et al., 2020; Stein et al., 2018; Zhai et al., 2011; Figure 1). This enabled us to date the emergence of the Pik pair to before the split of two major grass lineages: the BOP and PACMAD clades, which corresponds to 100-50 MYA (Hodkinson, 2018). Furthermore, we estimated that Pik-1 acquired the HMA domain prior to the emergence of Oryzinae but after the split from Panicoideae, between 15 and 100 MYA (Hodkinson, 2018; Jacquemin et al., 2011; Stein et al., 2018). Remarkably, the vast majority of Pik-2 and Pik-1 orthologues across the Poaceae exist as genetically linked pairs in a head-to-head orientation. This applies to Pik-1 orthologues with and without the HMA domain, indicating that Pik-1 and Pik-2 pairing occurred prior to HMA integration. Tight genetic linkage of paired NLRs, such as Pik-1/Pik-2 (Ashikawa et alı, 2008), RGA5/RGA4 (Cesari et al., 2013; Okuyama et al., 2011), RRS1/RPS4 (Saucet et al., 2015), or RPP2A/RPP2B (Sinapidou et al., 2004), is thought to facilitate coregulation and coevolution, thereby ensuring proper cooperation between these NLRs and reducing the genetic load caused by autoimmunity (Baggs et al., 2017; Griebel et al., 2014; Wu et al., 2018). However, Pik-1 and Pik-2 paralogues also occur adjacent to the paired genes-a phenomenon previously observed in wild and cultivated rice (Mizuno et al., 2020)_raising the possibility that these Pik genes may form an NLR receptor network beyond the Pik-1/Pik-2 pair (Wu et al., 2018). In the future, it would be interesting to investigate the functions of paired Pik-1/Pik-2 and their paralogues and determine whether functional pairing and genetic linkage with Pik-2 predisposed Pik-1 for the HMA integration.

In summary, our study illustrates the value of ASR-a method that has rarely been used in the field of plant-microbe interactions (Dong et al., 2014; Tanaka et al., 2019; Zess et al., 2019)—in transcending phylogenetic inference to yield a more elaborate evolutionary model. ASR combined with biochemical and biophysical studies enabled us to determine the directionality of evolution and therefore develop an experimentally validated model of NLR adaptation. The Pik-1/Pik-2 receptor pair emerged as an excellent system to not only provide a framework for drawing links between NLR structure and function but also to place this knowledge in an evolutionary context. This adds to our understanding of selection forces, historical contingency, and functional constraints shaping NLR activities. This approach illustrates how mechanistic research structured by a robust evolutionary framework can enhance our understanding of plant-microbe systems. 


\section{Materials and methods}

\section{Key resources table}

\begin{tabular}{|c|c|c|c|c|}
\hline $\begin{array}{l}\text { Reagent type } \\
\text { (species) or resource }\end{array}$ & Designation & $\begin{array}{l}\text { Source or } \\
\text { reference }\end{array}$ & Identifiers & Additional information \\
\hline $\begin{array}{l}\text { Recombinant } \\
\text { DNA reagent }\end{array}$ & $\mathrm{plCH} 41308$ & Addgene & No. 47998 & $\begin{array}{l}\text { Golden Gate } \\
\text { level } 0 \text { acceptor }\end{array}$ \\
\hline $\begin{array}{l}\text { Recombinant } \\
\text { DNA reagent }\end{array}$ & plCSL12008 & $\begin{array}{l}\text { TSL (The Sainsbury } \\
\text { Laboratory) SynBio } \\
\text { team }\end{array}$ & & $\begin{array}{l}35 \mathrm{~S}+\Omega \text { promoter Golden } \\
\text { Gate module }\end{array}$ \\
\hline $\begin{array}{l}\text { Recombinant } \\
\text { DNA reagent }\end{array}$ & $\mathrm{plCH} 41414$ & Addgene & No. 50337 & $\begin{array}{l}35 S \text { terminator Golden } \\
\text { Gate module }\end{array}$ \\
\hline $\begin{array}{l}\text { Recombinant } \\
\text { DNA reagent }\end{array}$ & pICSL30007 & $\begin{array}{l}\text { TSL (The Sainsbury } \\
\text { Laboratory) SynBio } \\
\text { team }\end{array}$ & & $\begin{array}{l}\text { N-terminal } 6 \times \mathrm{HA} \text { Golden } \\
\text { Gate module }\end{array}$ \\
\hline $\begin{array}{l}\text { Recombinant } \\
\text { DNA reagent }\end{array}$ & $\mathrm{plCH} 47732$ & Addgene & No. 48001 & Level 1 binary vector \\
\hline $\begin{array}{l}\text { Recombinant } \\
\text { DNA reagent }\end{array}$ & p41308-PikpN & This paper & & $\begin{array}{l}\text { Materials and methods: } \\
\text { Cloning for in planta assays }\end{array}$ \\
\hline $\begin{array}{l}\text { Recombinant } \\
\text { DNA reagent }\end{array}$ & p41308-PikpC & This paper & & $\begin{array}{l}\text { Materials and methods: } \\
\text { Cloning for in planta assays }\end{array}$ \\
\hline $\begin{array}{l}\text { Recombinant } \\
\text { DNA reagent }\end{array}$ & pICSL13004 & $\begin{array}{l}\text { TSL (The Sainsbury } \\
\text { Laboratory) SynBio } \\
\text { team }\end{array}$ & & $\begin{array}{l}\text { Mas promoter Golden } \\
\text { Gate module }\end{array}$ \\
\hline $\begin{array}{l}\text { Recombinant } \\
\text { DNA reagent }\end{array}$ & pICSL50001 & $\begin{array}{l}\text { TSL (The Sainsbury } \\
\text { Laboratory) SynBio } \\
\text { team }\end{array}$ & & $\begin{array}{l}\text { C-terminal HF Golden } \\
\text { Gate module }\end{array}$ \\
\hline $\begin{array}{l}\text { Recombinant } \\
\text { DNA reagent }\end{array}$ & $\mathrm{plCH} 77901$ & $\begin{array}{l}\text { TSL (The Sainsbury } \\
\text { Laboratory) SynBio } \\
\text { team }\end{array}$ & & $\begin{array}{l}\text { Mas terminator Golden } \\
\text { Gate module }\end{array}$ \\
\hline $\begin{array}{l}\text { Recombinant } \\
\text { DNA reagent }\end{array}$ & p41308-PikmN & This paper & & $\begin{array}{l}\text { Materials and methods: } \\
\text { Cloning for in planta assays }\end{array}$ \\
\hline $\begin{array}{l}\text { Recombinant } \\
\text { DNA reagent }\end{array}$ & p41308-PikmC & This paper & & $\begin{array}{l}\text { Materials and methods: } \\
\text { Cloning for in planta assays }\end{array}$ \\
\hline $\begin{array}{l}\text { Recombinant } \\
\text { DNA reagent }\end{array}$ & pOPIN-M & Addgene & No. 26044 & E. coli expression vector \\
\hline $\begin{array}{l}\text { Recombinant } \\
\text { DNA reagent }\end{array}$ & AVR-PikD in pOPIN-S3C & $\begin{array}{l}\text { Maqbool et al., } \\
2015\end{array}$ & & E. coli expression construct \\
\hline $\begin{array}{l}\text { Commercial } \\
\text { assay, kit }\end{array}$ & $\begin{array}{l}\text { Anti-HA Affinity Matrix, } \\
\text { from rat } \lg \mathrm{G} 1\end{array}$ & Roche & 11815016001 & $\begin{array}{l}\text { Materials and methods: } \\
\text { Protein-protein interaction } \\
\text { studies: co-IP; } 20 \mu \mathrm{L}\end{array}$ \\
\hline Antibody & $\begin{array}{l}\text { HA-probe (F-7) HRP-conjugated; } \\
\text { mouse monoclonal lgG2a }\end{array}$ & Santa Cruz Biotech & sc-7392 & $\begin{array}{l}\text { Materials and methods: } \\
\text { Protein-protein interaction } \\
\text { studies: co-IP; 1:5000 }\end{array}$ \\
\hline Antibody & $\begin{array}{l}\text { Mouse monoclonal } \\
\text { ANTI-FLAG M2 }\end{array}$ & Sigma & F3165 & $\begin{array}{l}\text { Materials and methods: } \\
\text { Protein-protein interaction } \\
\text { studies: co-IP }\end{array}$ \\
\hline Antibody & $\begin{array}{l}\text { A-14 anti-Myc antibody; } \\
\text { A-14 anti-Myc antibody }\end{array}$ & $\begin{array}{l}\text { Santa Cruz } \\
\text { Biotechnology }\end{array}$ & Sc-40 & $\begin{array}{l}\text { Materials and methods: } \\
\text { Protein-protein interaction } \\
\text { studies: co-IP; 1:5000 }\end{array}$ \\
\hline $\begin{array}{l}\text { Commercial } \\
\text { assay, kit }\end{array}$ & $\begin{array}{l}\text { Pierce ECL Western } \\
\text { Blotting Substrate }\end{array}$ & $\begin{array}{l}\text { Thermo } \\
\text { Fisher Scientific }\end{array}$ & 32109 & $\begin{array}{l}\text { Materials and methods: } \\
\text { Protein-protein interaction } \\
\text { studies: co-IP; 1:5000 }\end{array}$ \\
\hline $\begin{array}{l}\text { Commercial } \\
\text { assay, kit }\end{array}$ & $\begin{array}{l}\text { SuperSignal West Femto } \\
\text { Maximum Sensitivity Substrate }\end{array}$ & $\begin{array}{l}\text { Thermo } \\
\text { Fisher Scientific }\end{array}$ & 34094 & $\begin{array}{l}\text { Materials and methods: } \\
\text { Protein-protein interaction } \\
\text { studies: co-IP; 1:5000 }\end{array}$ \\
\hline
\end{tabular}

Continued on next page 
Continued

\section{Reagent type}

(species) or resource Designation

Commercial Pierce Reversible Protein

assay, kit
Source or

reference

Thermo

Fisher Scientific

\section{Identifiers Additional information}

24585

Materials and methods:

Protein-protein

interaction

studies: co-IP; 1:5000

Potterton et al., 2018

Materials and methods: Crystallisation, data collection, and structure solution

Software, algorithm MolProbity

Chen et al., 2010

McNicholas et al., 2011

\begin{tabular}{lll}
\hline Software, algorithm CCP4MG & $\begin{array}{l}\text { McNicholas et al., } \\
2011\end{array}$ & $\begin{array}{l}\text { Waterhouse et al., } \\
\text { Software, algorithm }\end{array}$ \\
& SWISS-MODEL & 2018
\end{tabular}

Software, algorithm besthr

MacLean, 2019

Steuernagel et al.,

\begin{tabular}{ll}
\hline Software, algorithm NLR-Parser & Steuer \\
& 2015
\end{tabular}

Software, algorithm HMMER 3.2b2

Eddy, 1998

Edgar, 2004

Software, algorithm MUSCLE v2.8.31

https://github.com/

matthewmoscou/

OKphylogeny

\begin{tabular}{lll}
\hline Software, algorithm & OKphylogeny & $\begin{array}{l}\text { https://github.co } \\
\text { matthewmoscou/ } \\
\text { OKphylogeny }\end{array}$ \\
\hline Software, algorithm & RAxML v8.2.11 & $\begin{array}{c}\text { Stamatakis, 20 } \\
\end{array}$ \\
\hline Software, algorithm & iTOL v5.5.1 & $\begin{array}{l}\text { Letunic and Bo } \\
\mathbf{2 0 0 7}\end{array}$ \\
\hline Software, algorithm & BLAST v2.3.0 & \\
\hline & & Altschul et al., \\
& & 1990
\end{tabular}

Software, algorithm MEGA X

Kumar et al., 2018

Ashkenazy et al.,

Software, algorithm FastML

2012

Software, algorithm PAML v4.9j

Yang, 1997

Software, algorithm ggplot2 R v3.6.3 package

Ginestet, 2011

Software, algorithm SNAP

https://www.hiv.lanl. gov/

Sequence-

based reagent

5'-TGAAGCAGATCCGAGACATAGCCT-3' This study

Sequence-

based reagent

5'-TACCCTGCTCCTGATTGCTGACT-3' This study

Materials and methods: and structure solution
Materials and methods:

Crystallisation, data collection, and structure solution

Materials and methods:

Crystallisation, data collection, and structure solution

Crystallisation, data collection,

Materials and methods:

Cell death assay

Materials and methods:

Identification and phylogenetic analysis of CC-NLRs from grasses

Materials and methods:

Identification and phylogenetic

analysis of CC-NLRs from grasses

Materials and methods:

Identification and phylogenetic

analysis of CC-NLRs from grasses

Materials and methods:

Identification and phylogenetic

analysis of CC-NLRs from grasses

Materials and methods:

Identification and phylogenetic analysis of CC-NLRs from grasses

Materials and methods:

Identification and phylogenetic

analysis of Pik-1 and

Pik-2 homologues

Materials and methods:

Phylogenetic analyses of rice

HMA domains and ancestral sequence reconstruction

Materials and methods:

Phylogenetic analyses of rice

HMA domains and ancestral sequence reconstruction

Materials and methods:

Testing for selection

Materials and methods:

Testing for selection

Materials and methods:

Testing for selection

Materials and methods:

Identification and cloning of Pik-1

and Pik-2 from Oryza brachyantha

Materials and methods:

Identification and cloning of Pik-1

and Pik-2 from Oryza brachyantha

\section{Continued on next page}


Continued

\begin{tabular}{|c|c|c|c|c|}
\hline $\begin{array}{l}\text { Reagent type } \\
\text { (species) or resource }\end{array}$ & Designation & $\begin{array}{l}\text { Source or } \\
\text { reference }\end{array}$ & Identifiers & Additional information \\
\hline $\begin{array}{l}\text { Sequence- } \\
\text { based reagent }\end{array}$ & 5'-AGGGAGCAATGATGCTTCACGA-3' & This study & PCR primer & $\begin{array}{l}\text { Materials and methods: } \\
\text { Identification and cloning of the } \\
\text { Pik-1-integrated HMA domains } \\
\text { from wild rice relatives }\end{array}$ \\
\hline $\begin{array}{l}\text { Sequence- } \\
\text { based reagent }\end{array}$ & 3'-TTCTCTGGCAACCGTTGTTTTGC-5' & This study & PCR primer & $\begin{array}{l}\text { Materials and methods: } \\
\text { Identification and cloning of the } \\
\text { Pik-1-integrated HMA domains } \\
\text { from wild rice relatives }\end{array}$ \\
\hline $\begin{array}{l}\text { Commercial } \\
\text { assay or kit }\end{array}$ & In-Fusion HD Cloning & Clontech & 639647 & $\begin{array}{l}\text { Materials and methods: } \\
\text { Cloning for in vitro studies }\end{array}$ \\
\hline $\begin{array}{l}\text { Gene } \\
\text { (O. brachyantha) }\end{array}$ & W0654 & $\begin{array}{l}\text { Wild Rice Collection } \\
\text { 'Oryzabase'; } \\
\text { Kurata and } \\
\text { Yamazaki, } 2006\end{array}$ & & $\begin{array}{l}\text { Materials and methods: } \\
\text { Identification and cloning of Pik-1 } \\
\text { and Pik-2 from Oryza brachyantha }\end{array}$ \\
\hline $\begin{array}{l}\text { Gene } \\
\text { (O. brachyantha) }\end{array}$ & W0655 & $\begin{array}{l}\text { Wild Rice Collection } \\
\text { 'Oryzabase'; } \\
\text { Kurata and } \\
\text { Yamazaki, } 2006\end{array}$ & & $\begin{array}{l}\text { Materials and methods: } \\
\text { Identification and cloning of Pik-1 } \\
\text { and Pik-2 from Oryza brachyantha }\end{array}$ \\
\hline $\begin{array}{l}\text { Gene } \\
\text { (O. brachyantha) }\end{array}$ & W0656 & $\begin{array}{l}\text { Wild Rice Collection } \\
\text { 'Oryzabase'; } \\
\text { Kurata and } \\
\text { Yamazaki, } 2006\end{array}$ & & $\begin{array}{l}\text { Materials and methods: } \\
\text { Identification and cloning of Pik-1 } \\
\text { and Pik-2 from Oryza brachyantha }\end{array}$ \\
\hline $\begin{array}{l}\text { Gene } \\
\text { (O. brachyantha) }\end{array}$ & W1057 & $\begin{array}{l}\text { Wild Rice Collection } \\
\text { 'Oryzabase'; } \\
\text { Kurata and } \\
\text { Yamazaki, } 2006\end{array}$ & & $\begin{array}{l}\text { Materials and methods: } \\
\text { Identification and cloning of Pik-1 } \\
\text { and Pik-2 from Oryza brachyantha }\end{array}$ \\
\hline $\begin{array}{l}\text { Gene } \\
\text { (O. brachyantha) }\end{array}$ & W1401 & $\begin{array}{l}\text { Wild Rice Collection } \\
\text { 'Oryzabase'; } \\
\text { Kurata and } \\
\text { Yamazaki, } 2006\end{array}$ & & $\begin{array}{l}\text { Materials and methods: } \\
\text { Identification and cloning of Pik-1 } \\
\text { and Pik-2 from Oryza brachyantha }\end{array}$ \\
\hline $\begin{array}{l}\text { Gene } \\
\text { (O. brachyantha) }\end{array}$ & W1402 & $\begin{array}{l}\text { Wild Rice Collection } \\
\text { 'Oryzabase'; } \\
\text { Kurata and } \\
\text { Yamazaki, } 2006\end{array}$ & & $\begin{array}{l}\text { Materials and methods: } \\
\text { Identification and cloning of Pik-1 } \\
\text { and Pik-2 from Oryza brachyantha }\end{array}$ \\
\hline $\begin{array}{l}\text { Gene } \\
\text { (O. brachyantha) }\end{array}$ & W1403 & $\begin{array}{l}\text { Wild Rice Collection } \\
\text { 'Oryzabase'; } \\
\text { Kurata and } \\
\text { Yamazaki, } 2006\end{array}$ & & $\begin{array}{l}\text { Materials and methods: } \\
\text { Identification and cloning of Pik-1 } \\
\text { and Pik-2 from Oryza brachyantha }\end{array}$ \\
\hline $\begin{array}{l}\text { Gene } \\
\text { (O. brachyantha) }\end{array}$ & W1404 & $\begin{array}{l}\text { Wild Rice Collection } \\
\text { 'Oryzabase'; } \\
\text { Kurata and } \\
\text { Yamazaki, } 2006\end{array}$ & & $\begin{array}{l}\text { Materials and methods: } \\
\text { Identification and cloning of Pik-1 } \\
\text { and Pik-2 from Oryza brachyantha }\end{array}$ \\
\hline $\begin{array}{l}\text { Gene } \\
\text { (O. brachyantha) }\end{array}$ & W1405 & $\begin{array}{l}\text { Wild Rice Collection } \\
\text { 'Oryzabase'; } \\
\text { Kurata and } \\
\text { Yamazaki, } 2006\end{array}$ & & $\begin{array}{l}\text { Materials and methods: } \\
\text { Identification and cloning of Pik-1 } \\
\text { and Pik-2 from Oryza brachyantha }\end{array}$ \\
\hline $\begin{array}{l}\text { Gene } \\
\text { (O. brachyantha) }\end{array}$ & W1407(B) & $\begin{array}{l}\text { Wild Rice Collection } \\
\text { 'Oryzabase'; } \\
\text { Kurata and } \\
\text { Yamazaki, } 2006\end{array}$ & & $\begin{array}{l}\text { Materials and methods: } \\
\text { Identification and cloning of Pik-1 } \\
\text { and Pik-2 from Oryza brachyantha }\end{array}$ \\
\hline $\begin{array}{l}\text { Gene } \\
\text { (O. brachyantha) }\end{array}$ & W1703 & $\begin{array}{l}\text { Wild Rice Collection } \\
\text { 'Oryzabase'; } \\
\text { Kurata and } \\
\text { Yamazaki, } 2006\end{array}$ & & $\begin{array}{l}\text { Materials and methods: } \\
\text { Identification and cloning of Pik-1 } \\
\text { and Pik-2 from Oryza brachyantha }\end{array}$ \\
\hline $\begin{array}{l}\text { Gene } \\
\text { (O. brachyantha) }\end{array}$ & W1705 & $\begin{array}{l}\text { Wild Rice Collection } \\
\text { 'Oryzabase'; } \\
\text { Kurata and } \\
\text { Yamazaki, } 2006\end{array}$ & & $\begin{array}{l}\text { Materials and methods: } \\
\text { Identification and cloning of Pik-1 } \\
\text { and Pik-2 from Oryza brachyantha }\end{array}$ \\
\hline
\end{tabular}

\section{Continued on next page}


Continued

Reagent type

(species) or resource Designation

Gene W1706

(O. brachyantha)

(O. brachyantha)

W1708

Gene W1708

Source or

Yamazaki, 2006

Gene W1711

(O. brachyantha)

Gene W1712

(O. brachyantha)

W1712

Gene

W0654

(O. brachyantha)

W0008

Gene

(O. australiensis)

Gene W1628

(O. australiensis)

W1628

Gene W1643

(O. barthii)

1643

Gene W1605

(O. barthii)

W1605

Gene W0042

(O. barthii)

N0042

Gene W0698

(O. barthii)

W0698

Gene W1526

(O. eichingeri)

W1526

'Oryzabase';
Kurata and

Gene W1171

(O. glumaepatula)

W1171

Gene W2203

(O. glumaepatula)

W2203

'Oryzabase';
Kurata and
Yamazaki, 2006

Gene W1480(B)

(O. grandiglumis) reference

Wild Rice Collection

'Oryzabase';

Kurata and

Wild Rice Collection

'Oryzabase';

Kurata and

Yamazaki, 2006

Yamazaki, 2006

\section{Identifiers Additional information}

Materials and methods:

Identification and cloning of Pik-1

and Pik-2 from Oryza brachyantha

Materials and methods:

Identification and cloning of Pik-1

and Pik-2 from Oryza brachyantha

Wild Rice Collection

'Oryzabase':

Kurata and

Yamazaki, 2006

Wild Rice Collection

'Oryzabase';

Kurata and

Yamazaki, 2006

Wild Rice Collection

'Oryzabase':

Yamazaki, 2006

Materials and methods:

Identification and cloning of Pik-1

and Pik-2 from Oryza brachyantha

Materials and methods:

Identification and cloning of Pik-1

and Pik-2 from Oryza brachyantha

Materials and methods:

Identification and cloning of the

Pik-1-integrated HMA domains

from wild rice relatives

Wild Rice Collection

'Oryzabase':

Kurata and

Yamazaki, 2006

Materials and methods:

Identification and cloning of the

Pik-1-integrated HMA domains

from wild rice relatives

Wild Rice Collection

'Oryzabase';

Kurata and

Materials and methods:

Identification and cloning of the

Pik-1-integrated HMA domains

from wild rice relatives

Wild Rice Collection

'Oryzabase':

Kurata and

Yamazaki, 2006

Materials and methods:

Identification and cloning of the

Pik-1-integrated HMA domains

from wild rice relatives

Wild Rice Collection

Materials and methods:

'Oryzabase';

Kurata and

Yamazaki, 2006

Identification and cloning of the

Pik-1-integrated HMA domains from wild rice relatives

Wild Rice Collection

'Oryzabase';

Yamazaki, 2006

Materials and methods:

Identification and cloning of the

Pik-1-integrated HMA domains

from wild rice relatives

Wild Rice Collection

'Oryzabase';

Kurata and

Yamazaki, 2006

Materials and methods:

Identification and cloning of the

Pik-1-integrated HMA domains from wild rice relatives

Materials and methods:

Identification and cloning of the

Pik-1-integrated HMA domains

from wild rice relatives

Wild Rice Collection

'Oryzabase':

Kurata and

Yamazaki, 2006

Materials and methods:

Identification and cloning of the

Pik-1-integrated HMA domains from wild rice relatives

Materials and methods:

Identification and cloning of the

Pik-1-integrated HMA domains from wild rice relatives

Wild Rice Collection

'Oryzabase':

Kurata and

Yamazaki, 2006

Materials and methods:

Identification and cloning of the

Pik-1-integrated HMA domains

from wild rice relatives

\section{Continued on next page}


Continued

Reagent type

(species) or resource Gene W0005

(O. granulata)

Gene W0067(B)

(O. granulata)

W0067(B)
Designation

$\begin{array}{ll}\text { Gene } & \text { W0542 } \\ \text { (O. latifolia/O. alta) } & \end{array}$

Source or

Gene W1539

(O. latifolia/O. alta)

$\begin{array}{ll} & \\ \text { Gene } & \text { W1228 }\end{array}$

Gene

N1228

Gene W1504

(O. longistaminata)

W1504

Gene W1540

(O. longistaminata)

W1540

Gene W0643

(O. longistaminata)

W0643

Gene W2081

(O. meridionalis)

W2081

Gene W2112

(O. meridionalis)

Gene W1354

(O. meyeriana)

(3)

Gene W1328

(O. minuta)

W1328

Gene W0614

(O. officinalis)

W0614

Gene W1200

(O. officinalis)

W1200

\begin{tabular}{|c|c|c|}
\hline (O. officinalis) & & $\begin{array}{l}\text { 'Oryzabase'; } \\
\text { Kurata and } \\
\text { Yamazaki, } 2006\end{array}$ \\
\hline $\begin{array}{l}\text { Gene } \\
\text { (O. punctata) }\end{array}$ & W1408 & $\begin{array}{l}\text { Wild Rice Collection } \\
\text { 'Oryzabase'; } \\
\text { Kurata and' } \\
\text { Yamazaki, } 2006\end{array}$ \\
\hline
\end{tabular}

\section{Continued on next page}

reference

Wild Rice Collection

'Oryzabase'.

Kurata and

Yamazaki, 2006

Wild Rice Collection

'Oryzabase';

Kurata and

Yamazaki, 2006

Wild Rice Collection

'Oryzabase';

Kurata and

Yamazaki, 2006

Identifiers Additional information

Materials and methods:

Identification and cloning of the

Pik-1-integrated HMA domains

from wild rice relatives

Materials and methods:

Identification and cloning of the

Pik-1-integrated HMA domains from wild rice relatives

Materials and methods:

Identification and cloning of the

Pik-1-integrated HMA domains

from wild rice relatives

Wild Rice Collection

'Oryzabase';

Kurata and

Yamazaki, 2006

Materials and methods:

Identification and cloning of the

Pik-1-integrated HMA domains from wild rice relatives

Wild Rice Collection

'Oryzabase';

Materials and methods:

Identification and cloning of the

Pik-1-integrated HMA domains

from wild rice relatives

Yamazaki, 2006

Materials and methods:

Identification and cloning of the

Pik-1-integrated HMA domains

from wild rice relatives

Yamazaki, 2006

Materials and methods:

Identification and cloning of the

Pik-1-integrated HMA domains

from wild rice relatives

Yamazaki, 2006

Materials and methods:

Identification and cloning of the

Pik-1-integrated HMA domains

from wild rice relatives

Yamazaki, 2006

Materials and methods:

Wild Rice Collection

'Oryzabase';

Kurata and

Yamazaki, 2006

Identification and cloning of the

Pik-1-integrated HMA domains

from wild rice relatives

Wild Rice Collection

'Oryzabase';

Kurata and

Yamazaki, 2006

Materials and methods:

Identification and cloning of the

Pik-1-integrated HMA domains

from wild rice relatives

Wild Rice Collection

'Oryzabase';

Materials and methods:

Identification and cloning of the

Pik-1-integrated HMA domains

Yamazaki, 2006

from wild rice relatives

Wild Rice Collection

'Oryzabase';

Kurata and

Yamazaki, 2006

Materials and methods:

Identification and cloning of the

Pik-1-integrated HMA domains

from wild rice relatives

Wild Rice Collection

'Oryzabase';

Kurata and

Yamazaki, 2006

Materials and methods:

Identification and cloning of the

Pik-1-integrated HMA domains from wild rice relatives

Materials and methods:

Identification and cloning of the

Pik-1-integrated HMA domains from wild rice relatives

Materials and methods:

Identification and cloning of the

Pik-1-integrated HMA domains

from wild rice relatives 
Continued

Reagent type

(species) or resource

Gene W1514

(O. punctata)

Gene
(O. rhizomatis)

(O. rhizomatis)

Gene W0001

(O. ridleyi)

$\begin{array}{ll} & \\ \text { Gene } & \text { W2035 } \\ \text { (O. ridleyi) } & \end{array}$

(O. ridleyi)

Gene W2003
(O. rufipogon) W

(O. rufipogon)

W1715

\begin{tabular}{ll}
\hline $\begin{array}{l}\text { Gene } \\
\text { (O. rufipogon) }\end{array}$ & W1715 \\
\hline Gene & W2117 \\
$\begin{array}{l}\text { (O. rufipogon/ } \\
\text { O. meridionalis) }\end{array}$ &
\end{tabular}

Gene LOC102699268

(O. brachyantha)

Gene OBART11G23150 GenBank

reference

GenBank
Source or

Wild Rice Collection

'Oryzabase'.

Kurata and

Yamazaki, 2006

Wild Rice Collection

'Oryzabase';

Kurata and

Yamazaki, 2006

Wild Rice Collection

'Oryzabase';

Kurata and

Yamazaki, 2006

Wild Rice Collection

'Oryzabase';

Kurata and

Yamazaki, 2006

Wild Rice Collection

'Oryzabase';

Kurata and

Yamazaki, 2006

Wild Rice Collection

'Oryzabase';

Kurata and

Yamazaki, 2006

Wild Rice Collection

'Oryzabase';

Kurata and

Yamazaki, 2006

Gene

(O. longistaminata)

KN541092.1

GenBank

GenBank

(O. punctata)

Gene

OPUNC11G19550

(O. sativa)

HM035360.1

GenBank

\begin{tabular}{lll}
\hline Gene & HM048900_1 & GenBank \\
(O. sativa) &
\end{tabular}

(O. sativa)

Gene HQ662330_1 GenBank

(O. sativa)

Gene HQ662329_1 GenBank

(O. sativa)

Gene AB462324.1 GenBank

(O. sativa)

\section{Gene}

(O. brachyantha)

LOC102708959

LOC102708959

GenBank
Identifiers Additional information

Materials and methods:

Identification and cloning of the

Pik-1-integrated HMA domains

from wild rice relatives

Materials and methods:

Identification and cloning of the

Pik-1-integrated HMA domains

from wild rice relatives

Materials and methods:

Identification and cloning of the

Pik-1-integrated HMA domains

from wild rice relatives

Materials and methods:

Identification and cloning of the

Pik-1-integrated HMA domains

from wild rice relatives

Materials and methods:

Identification and cloning of the

Pik-1-integrated HMA domains

from wild rice relatives

Materials and methods:

Identification and cloning of the

Pik-1-integrated HMA domains

from wild rice relatives

Materials and methods:

Identification and cloning of the

Pik-1-integrated HMA domains

from wild rice relatives

Materials and methods:

Phylogenetic analyses of rice HMA domains and ancestral sequence reconstruction

Materials and methods:

Phylogenetic analyses of rice HMA domains and ancestral sequence reconstruction

Materials and methods:

Phylogenetic analyses of rice HMA domains and ancestral sequence reconstruction

Materials and methods:

Phylogenetic analyses of rice HMA domains and ancestral sequence reconstruction

Materials and methods:

Phylogenetic analyses of rice HMA domains and ancestral sequence reconstruction

Materials and methods:

Phylogenetic analyses of rice HMA domains and ancestral sequence reconstruction

Materials and methods:

Phylogenetic analyses of rice HMA domains and ancestral sequence reconstruction

Materials and methods:

Phylogenetic analyses of rice HMA domains and ancestral sequence reconstruction

Materials and methods:

Phylogenetic analyses of rice HMA domains and ancestral sequence reconstruction

Materials and methods:

Phylogenetic analyses of rice HMA domains and ancestral sequence reconstruction

\section{Continued on next page}


Continued

Reagent type

(species) or resource Designation

Gene LOC102709146

(O. brachyantha)
Source or

reference

GenBank

\section{Identifiers Additional information}

Materials and methods:

Phylogenetic analyses of rice HMA domains and ancestral sequence reconstruction

Gene LOC102714171 GenBank

(O. brachyantha)

Materials and methods:

Phylogenetic analyses of rice HMA domains and ancestral sequence reconstruction

Gene LOC102716957 GenBank

(O. brachyantha)

LOC102717220 GenBank

\section{Gene}

LOC102717220

GenBank

(O. brachyantha)

LOC_Os04g39360

GenBank

(O. sativa)

Gene

LOC_Os04g39370

GenBank

(O. sativa)

Os04g0469000_01

GenBank

Gene

(O. sativa)

Os02g0585200

Gene

(O. sativa)

Os02g0584800_01

Gene Os02g0584800_01 GenBank

(O. sativa)

Os02g0584700_01

GenBank

Gene

(O. sativa)

Os04g0469300_01

GenBank

\section{Gene}

(O. sativa)

Os02g0585100

GenBank

Gene

(1)

Gene

Os02g0584600

GenBank

(O. sativa)

Gene OSJNBa0060P14.7_01

(O. sativa)

Gene Os04g0464100_01 GenBank

(O. sativa)

\section{Gene}

Os02g0582600

(O. sativa)

GenBank
Materials and methods:

Phylogenetic analyses of rice HMA domains and ancestral sequence reconstruction

Materials and methods:

Phylogenetic analyses of rice HMA domains and ancestral sequence reconstruction

Materials and methods:

Phylogenetic analyses of rice HMA domains and ancestral sequence reconstruction

Materials and methods:

Phylogenetic analyses of rice HMA domains and ancestral sequence reconstruction

Materials and methods:

Phylogenetic analyses of rice HMA domains and ancestral sequence reconstruction

Materials and methods:

Phylogenetic analyses of rice HMA domains and ancestral sequence reconstruction

Materials and methods:

Phylogenetic analyses of rice HMA domains and ancestral sequence reconstruction

Materials and methods:

Phylogenetic analyses of rice HMA domains and ancestral sequence reconstruction

Materials and methods:

Phylogenetic analyses of rice HMA domains and ancestral sequence reconstruction

Materials and methods:

Phylogenetic analyses of rice HMA domains and ancestral sequence reconstruction

Materials and methods:

Phylogenetic analyses of rice HMA domains and ancestral sequence reconstruction

Materials and methods:

Phylogenetic analyses of rice HMA domains and ancestral sequence reconstruction

Materials and methods:

Phylogenetic analyses of rice HMA domains and ancestral sequence reconstruction

Materials and methods:

Phylogenetic analyses of rice HMA domains and ancestral sequence reconstruction

\section{Identification and phylogenetic analysis of CC-NLRs from grasses}

NLR-parser (Steuernagel et al., 2015) was used to identify the NLR sequences from the predicted protein databases of eight representative grass species, Brachypodium distachyon, $\mathrm{O}$. brachyantha, Oryza sativa, S. bicolor, Triticum aestivum, Zea mays (downloaded from Ensembl Plants collection), and Hordeum vulgare and S. italica (downloaded from Phytozome v12.1 collection), listed in Supplementary file 1A. NLR sequences that were longer than 750 amino acid were screened for features of the NB-ARC and LRR domains, defined by the PF00931, PF00560, PF07725, PF13306, and PF13855 pfam models, using HMMER $3.2 b 2$ (Eddy, 1998); signatures of the coiled-coil domain 
were identified using 'motif16' and 'motif17' defined in NLR-parser. Protein sequences of NLRs that contained at least two of the above features were aligned using MUSCLE v2.8.31 (Edgar, 2004). The proteins comprising fewer than 60 amino acids $\mathrm{N}$ - and C-terminally of the NB-ARC domain, relative to the NB-ARC domain of Pikp-2 (Maqbool et al., 2015), were removed, as were sequences with less than $50 \%$ coverage across the alignment. The dataset was further filtered so that for each gene there was only one representative protein isoform-with the exception of sequences from $B$. distachyon and S. bicolor that did not carry gene identifiers. Filtering resulted in a final list of 3062 CC-NLRs (Supplementary file 2 ) that were amended with 35 known and functionally characterised NLR-type resistance proteins from grasses, added for reference (Supplementary file 1B).

The amino acid sequences corresponding to the NB-ARC domain of the identified NLRs were aligned using MUSCLE v2.8.31 (Edgar, 2004). The alignment positions with more than $30 \%$ data missing were removed from the alignment using QKphylogeny (Moscou, 2019; https://github.com/ matthewmoscou/OKphylogeny). This revealed a final alignment of 241 amino acids, which was used for a phylogenetic analysis. A ML phylogenetic tree was calculated using RAxML v8.2.11 (Stamatakis, 2014) with bootstrap values (Felsenstein, 1985) based on 1000 iterations and best-scoring JTT likelihood model (Jones et al., 1992) selected by automatic protein model assignment using the ML criterion. Best ML tree was mid-point rooted and visualised using Interactive Tree of Life (iTOL) tool v5.5.1 (Letunic and Bork, 2007). The relationships of 28 and 38 proteins that grouped with rice Pikp-1 and Pikp-2, respectively, were further validated as follows. Genetic loci and gene coordinates for each of those NLRs were inspected and, if required, manually reannotated; identifiers of manually reannotated genes were amended with '. $n$ ' suffix. For each gene, one splice version was selected and aligned using MUSCLE v2.8.31 (Edgar, 2004). The ML phylogenetic trees of Pik-1- and Pik-2related NLRs were calculated based on positions within the NB-ARC domain, for which more than $70 \%$ of data were present-957 and 1218 nucleotides for Pik-1 and Pik-2, respectively. The trees were generated using RAxML v8.2.11 (Stamatakis, 2014) with bootstrap values (Felsenstein, 1985) based on 1000 iterations and GTRGAMMA substitution model (Tavaré, 1986). Best ML trees were manually rooted based on the relationships observed in the above analyses and visualised using the iTOL tool v5.5.1 (Letunic and Bork, 2007).

\section{Identification and phylogenetic analysis of Pik-1 and Pik-2 homologues}

Coding sequences of representative Pik-1 and Pik-2 genes were used to identify Pik homologues from cDNA databases of Oryza barthii, Oryza longistaminata, Oryza punctata, Oryza glumeapatula, Oryza glaberrima, Oryza rufipogon, Oryza nivara, L. perrieri, Zizania latifolia, and Dactylis glomerata, listed in Supplementary file 1D, using BLAST v2.3.0 (Altschul et al., 1990). For each sequence with BLASTN E-value cutoff <0.01, genetic loci and gene coordinates were inspected and, if necessary, manually reannotated; identifiers of manually reannotated genes were amended with '. $n$ ' suffix. Because the Pik-1 and Pik-2 genes are known to be genetically linked, each Pik locus was further examined for signatures of unpredicted Pik gene candidates. Next, coding sequences of the Pik-1 and Pik-2 candidate homologues were aligned using MUSCLE v2.8.31 (Edgar, 2004). Poorly aligned sequences were manually removed from the alignment and excluded from further analysis. The phylogenetic trees were calculated based on positions within the NB-ARC domain, for which more than $70 \%$ of data was present-927 and 1239 nucleotides of 46 Pik-1 and 54 Pik-2 candidates, respectively. ML phylogenetic trees were calculated using RAxML v8.2.11 (Stamatakis, 2014) with bootstrap values based on 1000 iterations (Felsenstein, 1985) and GTRGAMMA substitution model (Tavaré, 1986). Best ML trees were manually rooted according to previously observed relationship and visualised using the iTOL tool v5.5.1 (Letunic and Bork, 2007).

\section{Phylogenetic analyses of rice HMA domains and ancestral sequence reconstruction}

Selected non-integrated HMA sequences from $O$. sativa and $O$. brachyantha were obtained by BLASTP search (Altschul et al., 1990) using Pikp-1 HMA (Pikp-HMA) as a query. Amino acid and nucleotide alignments were generated using MUSCLE (Edgar, 2004). NJ clustering method (Saitou and Nei, 1987) was used for constructing protein-based or codon-based trees based on JTT (Jones et al., 1992) or Maximum Composite Likelihood substitution models, respectively, using 1000 bootstrap tests (Felsenstein, 1985), as implemented in MEGA X (Kumar et al., 2018). ML 
trees were calculated using JTT (Jones et al., 1992) or GTR (Tavaré, 1986) substitution models as implemented in MEGA X software (Kumar et al., 2018).

Three independent protein sequence alignments, generated with MUSCLE (Edgar, 2004), were used for ASR (Supplementary file 1M). Joint and marginal ASRs were performed with FastML software (Ashkenazy et al., 2012) using JTT substitution model (Jones et al., 1992), gamma distribution, and $90 \%$ probability cutoff to prefer ancestral indel over a character. The reconstruction was performed based on NJ trees (Saitou and Nei, 1987) built with 100 iteration bootstrap method (Felsenstein, 1985). Sequences after marginal reconstruction including indels were used for further analyses.

\section{Testing for selection}

The rates of synonymous $\left(d_{S}\right)$ and nonsynonymous $\left(d_{N}\right)$ nucleotide substitutions per site in pairwise comparisons of protein-coding DNA sequences were estimated using the Yang and Nielsen, 2000 method under realistic evolutionary models, as implemented in the YN00 program in the PAML v4.9j package (Yang, 1997). The coding sequence alignments used for the analysis were generated using MUSCLE v2.8.31 (Edgar, 2004); unless stated otherwise, only positions that showed over $70 \%$ coverage across the alignment were used for the analyses.

For selection across the sites of the HMA domain, site models were implemented using the CODEML program in the PAML v4.9j software package (Yang, 1997). The three null models, M0 (one-ratio), M1 (nearly neutral), M7 (beta), and three alternative models, M3 (selection), M2 (discrete), M8 (beta and $\omega$ ), were tested as recommended by Yang et al., 2000, and their likelihoods were calculated with the LRT. The difference in log likelihood ratio between a null model and an alternative model was multiplied by 2 and compared with the chi-squared $\left(\chi^{2}\right)$ distribution; the degrees of freedom were calculated from the difference in the numbers of parameters estimated from the model pairs. The naïve empirical Bayes (NEB) (Yang, 2000; Yang and Nielsen, 1998) or the BEB (Yang et al., 2005) were used to infer the posterior probabilities for site classes and identify amino acids under positive selection. Raw data were extracted and visualised using the ggplot2 R v3.6.3 package (Ginestet, 2011). ML phylogenetic tree used for the analysis was built with bootstrap values (Felsenstein, 1985) from 1000 iterations using MEGA X software (Kumar et al., 2018), based on coding sequence alignment, generated with MUSCLE v2.8.31 (Edgar, 2004).

The pairwise rates of synonymous and nonsynonymous substitutions across Pik-1 allelic variants of rice were calculated using the Nei and Gojobori, 1986 method, as implemented using the SNAP tool (https://www.hiv.lanl.gov/).

\section{Identification and cloning of Pik-1 and Pik-2 from O. brachyantha}

Genomic DNA materials of $16 \mathrm{O}$. brachyantha accessions were ordered from Wild Rice Collection 'Oryzabase' (Supplementary file 1C; Kurata and Yamazaki, 2006). The accessions were first screened for deletion within the Pik-2 gene, present in a reference genome of $O$. brachyantha (Chen et al., 2013). Selected accessions were used to amplify full-length Pik-1 and Pik-2 genes using 5'-TGAAGCAGATCCGAGACATAGCCT-3' and 5'-TACCCTGCTCCTGATTGCTGACT-3' primers designed based on the $O$. brachyantha genome sequence (Chen et alı, 2013). The PCRs were run on agarose gels to check amplification and product size against positive controls. Fragments of the expected size were further gel purified, cloned into Zero Blunt TOPO plasmid (Thermo Fisher Scientific), and sequenced.

\section{Identification and cloning of the Pik-1-integrated HMA domains from wild rice relatives}

Genomic DNA materials of 1-3 accessions of 18 wild rice species-Oryza australiensis, O. barthii, $O$. brachyantha, Oryza eichingeri, Oryza glumaepatula, Oryza grandiglumis, Oryza granulata, Oryza latifolia, Oryza longiglumis, O. longistaminata, Oryza meridionalis, Oryza meyeriana, Oryza minuta, Oryza officinalis, O. punctata, Oryza rhizomatis, Oryza ridleyi, O. rufipogon-were ordered from Wild Rice Collection 'Oryzabase' (Kurata and Yamazaki, 2006) and used for amplification of the Pik-1-integrated HMA (Supplementary file 1H). The 5'-AGGGAGCAATGATGCTTCACGA-3' and 3'TTCTCTGGCAACCGTTGTTTTGC-5' primers were designed using the alignment of the OsPikp-1 and OBRAC11G13570.1 sequences and used in PCR. The amplicons were run on agarose gels to 
check amplification and product sizes against positive controls. Fragments of 450-720 bp in size were gel-purified, cloned into Zero Blunt TOPO plasmid (Thermo Fisher Scientific), and sequenced. Genotyping was performed twice, and only sequences that did not show ambiguity between sequencing runs were selected for further analyses.

\section{Cloning for in planta assays}

The rice Pikp-1, previously cloned by Maqbool et al., 2015, was amplified from pCambia1300:Ascl plasmid and domesticated to remove internal Bsal and Bpil restriction enzyme recognition sites using site-directed mutagenesis by inverse PCR. The amplicons were purified and assembled using the Golden Gate method (Weber et al., 2011) in the level 0 plCH41308 (Addgene no. 47998) destination vector for subsequent Golden Gate cloning. The N-terminally tagged HA:Pikp-1 expression construct was generated by Golden Gate assembly with pICSL12008 (35S + $\Omega$ promoter, TSL SynBio), pICSL30007 (N-terminal 6×HA, TSL SynBio), and plCH41414 (35S terminator, Addgene no. 50337) modules, into the binary vector plCH47732 (Addgene no. 48001). Using the same set of Golden Gate modules, Pikp-1 $1_{\mathrm{E} 230 \mathrm{R}}$ mutant was subcloned into the same binary vector, generating the N-terminally tagged HA:Pikp-1 $1_{\mathrm{E} 230 \mathrm{R}}$ expression construct.

The ancHMA variants-corresponding to 186-260 residues of the full-length Pikp-1-were synthesised as level 0 modules for Golden Gate cloning by GENEWIZ (South Plainfield, NJ, USA). Cloning of subsequent Pikp-1:ancHMA fusions was done using two custom-made Golden Gate level 0 acceptor plasmids, p41308-PikpN and p41308-PikpC, that allowed HMA insertion in a single Golden Gate level 0 reaction, generating full-length Pikp- 1 constructs with or without a stop codon, respectively. The ancHMA mutants-ancHMA $A_{A M E G N N D,}$ ancHMA $A_{L Y}$, ancHMA $A_{P I}$, ancHMA $A_{L V I E}$, and the single mutants within the LVKIE region of the ancHMA-were synthesised by GENEWIZ and subcloned into p41308-PikpN and p41308-PikpC plasmids for cloning. Two of the ancHMA mutants, ancHMA IVOVE and ancHMA $A_{L V K I V}$, were generated using site-directed mutagenesis by inverse PCR and cloned into the same acceptor plasmids. Using the p41308-PikpN modules, HA:Pikp-1:ancHMA expression constructs were generated by Golden Gate assembly with pICSL12008 (35S + $\Omega$ promoter, TSL SynBio), pICSL30007 (N-terminal 6×HA, TSL SynBio), and pICH41414 (35S terminator, Addgene no. 50337) into the binary vector plCH47732 (Addgene no. 48001). To generate C-terminally tagged expression constructs, the p41308-PikpC modules were assembled with pICSL13004 (Mas promoter, TSL SynBio), pICSL50001 (C-terminal HF, TSL SynBio), and pICH77901 (Mas terminator, TSL SynBio) by Golden Gate method into the same binary vector.

To generate Pikm-1:ancHMA fusions, ancHMA N2-I, ancHMA $A_{E M V K E}$, ancHMA $A_{F F E}$, ancHMA ancHMA $_{V H}$, and ancHMA IVDPM were synthesised by GENEWIZ as Golden Gate modules. The ancHMA EMANK $_{\text {mutant }}$ was generated by amplification and fusion of the $\mathrm{N}$-terminus of ancHMA $_{E M V K E}$ construct and the C-terminus of N2-I ancHMA variant. All ancHMA constructs corresponded to 187-264 residues of the full-length Pikm-1 protein and were subsequently assembled with custom-made p41308-PikmN (TSL SynBio) or p41308-PikmC (TSL SynBio) level 0 acceptors to generate Pikm-1:ancHMA fusions with or without a stop codon, respectively. Obtained modules were then used to generate Pikm-1:ancHMA expression constructs, featuring either N-terminal HA of C-terminal HF tags, by Golden Gate assembly using the same set of modules as previously used for Pikp-1 and plCH47732 binary vector.

\section{Cloning for in vitro studies}

The ancHMA mutants were amplified from Golden Gate level 0 modules by PCR and cloned into pOPIN-M vector featuring N-terminal $6 x \mathrm{His}$ and MBP tags with a $3 \mathrm{C}$ protease cleavage site using InFusion cloning (Berrow et alı, 2007). The AVR-PikD used for crystallography was cloned into pOPIN-S3C featuring N-terminal 6xHis and SUMO tags with a $3 \mathrm{C}$ protease cleavage site using InFusion reaction. AVR-PikD used for SPR studies was cloned previously (Maqbool et al., 2015).

\section{Protein-protein interaction studies: co-IP}

The co-IP protocol was described previously (Win et al., 2011). Transient gene expression in planta was conducted by delivering T-DNA constructs within Agrobacterium tumefaciens strain GV3101:: pMP90 into $N$. benthamiana leaves, and the leave tissue was collected 3 days after infiltration. Co-IP was performed using affinity chromatography with anti-HA Affinity Matrix (Roche). After co-IP and 
washing, the beads were resuspended in $30 \mu \mathrm{L}$ of loading dye and eluted by incubating at $70^{\circ} \mathrm{C}$ for $10 \mathrm{~min}$. Proteins were separated by SDS-PAGE and transferred onto a polyvinylidene difluoride (PVDF) membrane using a Trans-Blot turbo transfer system (Bio-Rad). The membrane was blocked with $5 \%$ non-fat dried milk powder in Tris-buffered saline and 1\% Tween 20 and probed with appropriate antisera. HA-probe (F-7) horseradish peroxidase (HRP)-conjugated (Santa Cruz Biotech) was used for a single-step detection of HA tag. FLAG detection was carried using monoclonal ANTIFLAG M2 (Sigma) and anti-mouse HRP-conjugated antibodies in a two-step FLAG detection. A twostep detection of Myc was performed using anti-Myc (A-14, Santa Cruz Biotechnology) and anti-rabbit HRP-conjugated antibodies. Pierce ECL Western Blotting Substrate (Thermo Fisher Scientific) or SuperSignal West Femto Maximum Sensitivity Substrate (Thermo Fisher Scientific) were used for detection. Membranes were imaged using ImageQuant LAS 4000 luminescent imager (GE Healthcare Life Sciences). Equal loading was checked by staining PVDF membranes with Pierce Reversible Protein Stain Kit (Thermo Fisher Scientific), Ponceau S, or Coomassie Brilliant Blue staining solutions.

\section{Protein-protein interaction studies: SPR}

SPR experiments to investigate the effects of the IAQVV/LVKIE and MKANK/EMVKE regions were performed in the SPR buffer 1 (50 mM HEPES, pH 7.5; $300 \mathrm{mM} \mathrm{NaCl}$; and 0.1\% Tween 20) and SPR buffer 2 (50 mM HEPES, pH 7.5; $820 \mathrm{mM} \mathrm{NaCl}$; and 0.1\% Tween 20), respectively, at $25^{\circ} \mathrm{C}$ using Biacore T200 (GE Healthcare). The 6xHis-tagged AVR-PikD (ligand) was immobilised on the Series S Sensor Chip NTA (GE Healthcare) and the HMA constructs (analytes) flowed over the effector at a flow rate of $30 \mu \mathrm{L} / \mathrm{min}$. For each cycle, the chip was washed with the appropriate SPR buffer and activated with $30 \mu \mathrm{L}$ of $0.5 \mathrm{mM} \mathrm{NiCl}$ prior to immobilisation of AVR-PikD. The HMA proteins were injected over both reference and sample cells at a range of concentrations for $120 \mathrm{~s}$, and buffer only flowed for $120 \mathrm{~s}$ to record the dissociation. Between each cycle, the sensor chip was regenerated with $30 \mu \mathrm{L}$ of $0.35 \mathrm{M}$ EDTA. To correct for bulk refractive index changes or machine errors, for each measurement the response was subtracted by the response in the reference cell and the response in buffer-only run (Myszka, 1999). The resulting sensorgrams were analysed using the Biacore Insight Evaluation Software (GE Healthcare).

The theoretical maximum responses $\left(R_{\max }\right)$ normalised for the amount of ligand immobilised on the chip were calculated, and the level of binding was expressed as a percentage of $R_{\max }\left(\% R_{\max }\right)$. Each experiment was repeated a minimum of three times. The data were visualised using ggplot2 $\mathrm{R}$ package (Ginestet, 2011).

\section{Heterologous protein production and purification}

Heterologous production and purification of ancHMA were performed as previously described (Varden et alı, 2019). AVR-PikD and ancHMA proteins used for purification were expressed in pOPIN-S3C and pOPIN-M plasmids, respectively. AVR-PikD effector with non-cleavable C-terminal 6xHis tag, used in SPR, was produced and purified as previously described (Maqbool et al., 2015). Protein intact masses were measured by static infusion of samples desalted by acetone precipitation and dissolved in $0.2 \%$ formic acid in $30 \%$ acetonitrile on Orbitrap Fusion (Thermo Scientific, UK). Data were acquired in a positive mode at 240,000 resolution and 1.6-2 kV spray voltage. The selected spectra were deisotoped and deconvoluted with Xtract software integrated in the Xcalibur package (Thermo Scientific).

\section{Crystallisation, data collection, and structure solution}

Crystallisation screens were performed at $18^{\circ} \mathrm{C}$ using the sitting-drop vapour diffusion technique. Drops composed of $0.3 \mu \mathrm{L}$ of protein solution and $0.3 \mu \mathrm{L}$ of reservoir solution were set up in MRC 96-well crystallisation plates (Molecular Dimensions), which were dispensed using an Oryx Nano or an Oryx8 robot (Douglas Instruments). Crystal growth was monitored using a Minstrel Desktop Crystal Imaging System (Rikagu). We attempted crystallisation of the ancHMA, ancHMA $A_{L V K I E}$, and ancHMA $_{E M V K E}$ domains in complexes with AVR-PikD, but only obtained diffracting crystals for ancHMA $_{\text {LVKIE }}-A V R-P i k D$. These crystals grew after $24-48 \mathrm{hr}$ in $14 \%(\mathrm{w} / \mathrm{v})$ PEG 3350 and $0.2 \mathrm{M}$ trisodium citrate and were harvested into a cryoprotectant comprising the precipitant augmented with $25 \%(v / v)$ ethylene glycol before flash-cooling in liquid nitrogen using LithoLoops (Molecular Dimensions). X-ray datasets were collected at the Diamond Light Source using beamline I03 (Didcot, UK) 
using a Pilatus3 6M hybrid photon counting detector (Dectris), with crystals maintained at $100 \mathrm{~K}$ by a Cryojet cryocooler (Oxford Instruments).

X-ray datasets were integrated and scaled using the DIALS xia2 pipeline (Winter, 2010) and merged with AIMLESS (Evans and Murshudov, 2013) implemented in the CCP4i2 graphical user interface (Potterton et al., 2018), with the best dataset being processed to $1.32 \AA$ resolution in space group $P 4{ }_{1} 2{ }_{1} 2$ with cell parameters $a=b=119.5 \AA, c=36.0 \AA$. Since the latter was isomorphous to the HMA-AVR-PikD complex previously solved (PDB accession code 5A6W, Maqbool et al., 2015), a high-quality preliminary model could straightforwardly be obtained by direct refinement of the latter against the new dataset using REFMAC5 (Murshudov et al., 2011). The asymmetric unit of this preliminary model comprised one copy of AVR-PikD and two copies of ancHMA $A_{\text {LVIE. }}$. The sequences of the latter chains were subsequently corrected by manually editing the model in COOT (Emsley et al., 2010). This model was finalised by iterative rounds of manual rebuilding in COOT (Emsley et al., 2010) and restrained refinement with anisotropic thermal parameters in REFMAC5 (Murshudov et al., 2011). The resultant structure was assessed with the tools provided in COOT and MolProbity (Chen et al., 2010) and visualised using CCP4MG software (McNicholas et al., 2011).

\section{Homology modelling}

Homology modelling of the ancHMA structure in complex with AVR-PikD was built using SWISSMODEL (Waterhouse et al., 2018) using coordinates of Pikm-HMA-AVR-PikD structure (PDB accession 6fu9) as a template.

\section{Cell death assay}

Expression constructs and conditions used for cell death/HR assay are listed in Supplementary file $1 N$. Transient expression in $N$. benthamiana leaves was conducted as previously described (Bos et al., 2006). Briefly, GV3101::pM90 A. tumefaciens strains carrying the appropriate expression vectors were mixed and resuspended in infiltration buffer $(10 \mathrm{mM} 2$-[N-morpholine]-ethanesulfonic acid [MES]; $10 \mathrm{mM} \mathrm{MgCl}$; and $150 \mu \mathrm{M}$ acetosyringone, $\mathrm{pH}$ 5.6) to a desired density. Upper leaves of 4-5-week-old $N$. benthamiana plants were used for infiltration. The HR cell death was scored 5 days after agroinfiltration using a previously published scale (Segretin et al., 2014) modified to range from 0 (no visible necrosis) to 7 (confluent necrosis).

\section{Acknowledgements}

We are thankful to several colleagues, in particular members of the Kamoun and Banfield labs, for discussions, ideas, and support. We thank Dan MacLean and other members of the TSL Bioinformatics team and Mark Youles of TSL SynBio for invaluable technical support. We also thank the Diamond Light Source, UK (beamline I03 under proposal MX18565), for access to X-ray data collection facilities and Andrew Davis and Phil Robinson of JIC Bioimaging facilities for photography. This work was supported by the Gatsby Charitable Foundation, Biotechnology and Biological Sciences Research Council (BBSRC, UK), the European Research Council (ERC grant: proposal 743165) (SK, MJB), and BBSRC Doctoral Training Partnership at Norwich Research Park (grant: BB/M011216/1, project reference: 1771322) (AB). MJB receives funding from BBSRC (BB/P012574, BB/M02198X) and the John Innes Foundation.

\section{Additional information}

Competing interests

Sophien Kamoun: receives funding from industry on NLR biology. The other authors declare that no competing interests exist.

Funding

Funder Grant reference number Author

Gatsby Charitable Foundation Sophien Kamoun 


\begin{tabular}{lll}
$\begin{array}{l}\text { Biotechnology and Biological } \\
\text { Sciences Research Council }\end{array}$ & Sophien Kamoun \\
\hline $\begin{array}{l}\text { Biotechnology and Biological } \\
\text { Sciences Research Council }\end{array}$ & BB/P012574 & Mark J Banfield \\
\hline $\begin{array}{l}\text { Biotechnology and Biological } \\
\text { Sciences Research Council }\end{array}$ & BB/M02198X & Mark J Banfield \\
\hline $\begin{array}{l}\text { European Research Council } \\
\text { Biotechnology and Biological }\end{array}$ & BB/M011216/1; 1771322 & $\begin{array}{l}\text { Mleksandra Białas } \\
\text { Sciences Research Council }\end{array}$ \\
\hline John Innes Foundation & & Mark J Banfield \\
\hline
\end{tabular}

The funders had no role in study design, data collection and interpretation, or the decision to submit the work for publication.

Author contributions

Aleksandra Białas, Conceptualization, Resources, Data curation, Formal analysis, Supervision, Funding acquisition, Investigation, Visualization, Methodology, Writing - original draft; Thorsten Langner, Conceptualization, Supervision, Methodology, Writing - original draft; Adeline Harant, Investigation; Mauricio P Contreras, Investigation, Writing - original draft; Clare EM Stevenson, David M Lawson, Jan Sklenar, Data curation, Formal analysis, Methodology, Writing - original draft; Ronny Kellner, Methodology; Matthew J Moscou, Resources, Methodology, Writing - original draft; Ryohei Terauchi, Resources, Funding acquisition, Methodology; Mark J Banfield, Resources, Supervision, Funding acquisition, Writing - original draft; Sophien Kamoun, Conceptualization, Resources, Supervision, Funding acquisition, Visualization, Writing - original draft, Project administration

Author ORCIDs

Aleksandra Białas (iD) https://orcid.org/0000-0002-1135-2189

Thorsten Langner (D) https://orcid.org/0000-0002-3401-8888

Mauricio P Contreras (iD https://orcid.org/0000-0001-6002-0730

David M Lawson (iD http://orcid.org/0000-0002-7637-4303

Ronny Kellner (iD http://orcid.org/0000-0002-4618-0110

Matthew J Moscou (D) https://orcid.org/0000-0003-2098-6818

Mark J Banfield (iD) http://orcid.org/0000-0001-8921-3835

Sophien Kamoun (iD https://orcid.org/0000-0002-0290-0315

Decision letter and Author response

Decision letter https://doi.org/10.7554/eLife.66961.sa1

Author response https://doi.org/10.7554/eLife.66961.sa2

\section{Additional files}

Supplementary files

- Supplementary file 1. Supplementary tables A-N.

- Supplementary file 2. Full list of all Poaceae NLRs and filtering details.

- Supplementary file 3. Site selection test for K-type HMAs.

- Supplementary file 4. ancHMA prediction probabilities.

- Transparent reporting form

Data availability

Diffraction data have been deposited in PDB under the accession code 7BNT. Sequencing data have been deposited in GenBank under accession codes: MW553204-MW553215 and MW568030MW568049. All relevant data generated or analysed during this study are included in the manuscript and supporting files. Source data files have been provided for Figure 1-source data 1, Figure 2- 
source data 1, Figure 5-source data 1, Figure 6-source data 1, Figure 6-figure supplement 1source data, Figure 6-Figure supplement 3-source data 1, Figure 8-source data 1.

The following previously published datasets were used:

\begin{tabular}{llll}
\hline Author(s) & Year Dataset title & Dataset URL & Database and \\
Identifier
\end{tabular}

International Brachypodium Initiative2018Genome sequencing and analysis of the model grass Brachypodium distachyonhttps://plants.ensembl.org/Brachypodium_distachyon/Info/Annotation/Ensembl Plants, GCA_00000550 5.4Mascher M, Gundlach H, Himmelbach A, Beier S, Twardziok SO, Wicker T, RadchukV, Dockter C, Hedley PE, Russell J, Bayer M, Ramsay L, Liu H, Haberer G, ZhangXQ, Zhang Q, Barrero RA, Li L, Taudien S, Groth M, Felder M, Hastie A, ŠimkováH, Staňková H, Vrána J, Chan S, Muñoz-Amatriaín M, Ounit R, Wanamaker S, BolserD, Colmsee C, Schmutzer T, Aliyeva-Schnorr L, Grasso S, Tanskanen J, Chailyan A, Sampath D, Heavens D, Clissold L, Cao S, Chapman B, Dai F, Han Y, Li H, Li X, Lin C, McCooke JK, Tan C, Wang P, Wang S, Yin S, Zhou G, Poland JA, Bellgard MI, Borisjuk L, Houben A, Doležel J, Ayling S, Lonardi S, Kersey P, Langridge P, Muehlbauer GJ, Clark MD, Caccamo M, Schulman AH, Mayer KFX, Platzer M, Close TJ, Scholz U, Hansson M, Zhang G, Braumann I, Spannagl M, Li C, Waugh R, Stein N2019A chromosome conformation capture ordered sequence of the barley genomehttps://plants.ensembl.org/Hordeum_vulgare/Info/Annotation/Ensembl Plants, GCA_901482405.1 Chen J, Huang Q, Gao D, Wang J, Lang Y, Liu T, Li B, Bai Z, Luis G, Liang C, Chen $C$, Zhang W, Sun S, Liao Y, Zhang X, Yang L, Song C, Wang M, ShiJ, Liu G, Liu J, Zhou H, Zhou W, Yu Q, An N, Chen Y, Cai Q, Wang B, Liu B, MinJ, Huang Y, Wu H, Li Z, Zhang Y, Yin Y, Song W, Jiang J, Jackson SA, Wing RA2012Whole-genome sequencing of Oryza brachyantha reveals mechanisms underlying Oryza genome evolutionhttps://plants.ensembl.org/Oryza_brachyantha/Info/Annotation/Ensembl Plants, GCA_0002310 95.2 Kawahara Y, de la Bastide M, Hamilton JP, Kanamori H, McCombie WR, Ouyang S, Schwartz DC, Tanaka T, Wu J, Zhou S, Childs KL, Davidson RM, Lin H, Quesada-Ocampo L, Vaillancourt B, Sakai H, Lee SS, Kim J, Numa H, Itoh T, Buell CR, Matsumoto T2015Improvement of the Oryza sativa Nipponbare reference genome using next generation sequence and optical map datahttps://plants.ensembl.org/Oryza_sativa/Info/ AnnotationEnsembl Plants, GCA_001433935.1 Bennetzen JL, Schmutz J, Wang H, Percifield R, Hawkins J, Pontaroli AC, Estep M, Feng L, Vaughn JN, Grimwood J, Jenkins J, Barry K, Lindquist E, Hellsten U, Deshpande S, Wang X, WuX, Mitros T, Triplett J, Yang X, Ye CY, Mauro-Herrera M, Wang L, Li P, Sharma M, Sharma R, Ronald PC, Panaud O, Kellogg EA, Brutnell TP, Doust AN, Tuskan GA, Rokhsar D, Devos KM20 15Reference genome sequence of the model plant Setariahttps://phytozome.jgi.doe.gov/pz/portal.html\#! info? alias=Org_SitalicaPhytozome v12.1, AGNK01000000.1 Saski C, Lee SB, Fjellheim S, Guda C, Jansen RK, Luo H, Tomkins J, Rognli OA, Daniell H, Clarke J2017Complete chloroplast genome sequences of Hordeum vulgare, Sorghum bicolor and Agrostis stolonifera, and comparative analyses with other grass genomeshttps:// plants.ensembl.org/Sorghum_bicolor/Info/Annotation/Ensembl Plants, GCA_000003195.3 International Wheat Genome Sequencing Consortium (IWGSC), International Wheat Genome Sequencing Consortium (IWGSC)20 18Bread wheat variety Chinese Spring for generating IWGSC RefSeq v1.0 assemblyhttps://plants.ensembl.org/ Triticum_aestivum/Info/Annotation/Ensembl Plants, GCA_900519105.1 Jiao Y, Peluso P, Shi J, Liang T, Stitzer MC, Wang B, Campbell MS, Stein JC, Wei X, Chin CS, Guill K, Regulski M, Kumari S, Olson A, Gent J, Schneider KL, Wolfgruber TK, May MR, Springer NM, Antoniou E, McCombie WR, Presting GG, McMullen M, Ross-lbarra J, Dawe RK, Hastie A, Rank DR, Ware D2017Improved maizereference genome with single-molecule technologieshttps://plants.ensembl.org/Zea_mays/Info/AnnotationEnsembl Plants, GCA_000005005.

\section{References}

Adachi H, Derevnina L, Kamoun S. 2019. NLR singletons, pairs, and networks: evolution, assembly, and regulation of the intracellular immunoreceptor circuitry of plants. Current Opinion in Plant Biology 50:121-131. DOI: https://doi.org/10.1016/j.pbi.2019.04.007, PMID: 31154077

Alcázar R, García AV, Parker JE, Reymond M. 2009. Incremental steps toward incompatibility revealed by Arabidopsis epistatic interactions modulating salicylic acid pathway activation. PNAS 106:334-339. DOI: https://doi.org/10.1073/pnas.0811734106, PMID: 19106299

Altschul SF, Gish W, Miller W, Myers EW, Lipman DJ. 1990. Basic local alignment search tool. Journal of Molecular Biology 215:403-410. DOI: https://doi.org/10.1016/S0022-2836(05)80360-2, PMID: 2231712

Ashikawa I, Hayashi N, Yamane H, Kanamori H, Wu J, Matsumoto T, Ono K, Yano M. 2008. Two adjacent nucleotide-binding site-leucine-rich repeat class genes are required to confer Pikm-specific rice blast resistance. Genetics 180:2267-2276. DOI: https://doi.org/10.1534/genetics.108.095034, PMID: 18940787

Ashkenazy H, Penn O, Doron-Faigenboim A, Cohen O, Cannarozzi G, Zomer O, Pupko T. 2012. FastML: a web server for probabilistic reconstruction of ancestral sequences. Nucleic Acids Research 40:W580-W584. DOI: https://doi.org/10.1093/nar/gks498

Baggs E, Dagdas G, Krasileva KV. 2017. NLR diversity, helpers and integrated domains: making sense of the NLR IDentity. Current Opinion in Plant Biology 38:59-67. DOI: https://doi.org/10.1016/j.pbi.2017.04.012, PMID: 2 8494248

Bailey PC, Schudoma C, Jackson W, Baggs E, Dagdas G, Haerty W, Moscou M, Krasileva KV. 2018. Dominant integration locus drives continuous diversification of plant immune receptors with exogenous domain fusions. Genome Biology 19:23. DOI: https://doi.org/10.1186/s13059-018-1392-6, PMID: 29458393

Bao J, Chen M, Zhong Z, Tang W, Lin L, Zhang X, Jiang H, Zhang D, Miao C, Tang H, Zhang J, Lu G, Ming R, Norvienyeku J, Wang B, Wang Z. 2017. PacBio sequencing reveals transposable elements as a key contributor to genomic plasticity and virulence variation in Magnaporthe oryzae. Molecular Plant 10:1465-1468. DOI: https://doi.org/10.1016/j.molp.2017.08.008, PMID: 28838703 
Bentham AR, Petit-Houdenot Y, Win J. 2021. A single amino acid polymorphism in a conserved effector of the multihost blast fungus pathogen expands host-target binding spectrum. bioRxiv. DOI: https://doi.org/10.1101/ 2021.03.15.435478

Berrow NS, Alderton D, Sainsbury S, Nettleship J, Assenberg R, Rahman N, Stuart DI, Owens RJ. 2007. A versatile ligation-independent cloning method suitable for high-throughput expression screening applications. Nucleic Acids Research 35:e45. DOI: https://doi.org/10.1093/nar/gkm047, PMID: 17317681

Bialas A, Zess EK, De la Concepcion JC, Franceschetti M, Pennington HG, Yoshida K, Upson JL, Chanclud E, Wu CH, Langner T, Maqbool A, Varden FA, Derevnina L, Belhaj K, Fujisaki K, Saitoh H, Terauchi R, Banfield MJ, Kamoun S. 2018. Lessons in effector and NLR biology of Plant-Microbe systems. Molecular Plant-Microbe Interactions 31:34-45. DOI: https://doi.org/10.1094/MPMI-08-17-0196-FI, PMID: 29144205

Białas A, Langner T, Banfield MJ, Kamoun S. 2021. Mutations That Abolish Pikm-1:anchma Autoactivity Are Inadequate for Testing the Impact of AVR-PikD Binding on Pikm-Mediated Immunity. Zenodo. 1. http://doi.org/ 10.5281/zenodo.4770746

Bomblies K, Lempe J, Epple P, Warthmann N, Lanz C, Dangl JL, Weigel D. 2007. Autoimmune response as a mechanism for a Dobzhansky-Muller-type incompatibility syndrome in plants. PLOS Biology 5:e236.

DOI: https://doi.org/10.1371/journal.pbio.0050236, PMID: 17803357

Bonardi V, Tang S, Stallmann A, Roberts M, Cherkis K, Dangl JL. 2011. Expanded functions for a family of plant intracellular immune receptors beyond specific recognition of pathogen effectors. PNAS 108:16463-16468. DOI: https://doi.org/10.1073/pnas.1113726108

Bos JI, Kanneganti TD, Young C, Cakir C, Huitema E, Win J, Armstrong MR, Birch PR, Kamoun S. 2006. The C-terminal half of Phytophthora infestans RXLR effector AVR3a is sufficient to trigger R3a-mediated hypersensitivity and suppress INF1-induced cell death in Nicotiana benthamiana. The Plant Journal 48:165-176. DOI: https://doi.org/10.1111/j.1365-313X.2006.02866.x, PMID: 16965554

Cesari S, Thilliez G, Ribot C, Chalvon V, Michel C, Jauneau A, Rivas S, Alaux L, Kanzaki H, Okuyama Y, Morel JB, Fournier E, Tharreau D, Terauchi R, Kroj T. 2013. The rice resistance protein pair RGA4/RGA5 recognizes the Magnaporthe oryzae effectors AVR-Pia and AVR1-CO39 by direct binding. The Plant Cell 25:1463-1481. DOI: https://doi.org/10.1105/tpc.112.107201, PMID: 23548743

Cesari S, Bernoux M, Moncuquet P, Kroj T, Dodds PN. 2014a. A novel conserved mechanism for plant NLR protein pairs: the "integrated decoy" hypothesis. Frontiers in Plant Science 5:606. DOI: https://doi.org/10. 3389/fpls.2014.00606, PMID: 25506347

Cesari S, Kanzaki H, Fujiwara T, Bernoux M, Chalvon V, Kawano Y, Shimamoto K, Dodds P, Terauchi R, Kroj T. 2014b. The NB-LRR proteins RGA4 and RGA5 interact functionally and physically to confer disease resistance. The EMBO Journal 33:1941-1959. DOI: https://doi.org/10.15252/embj.201487923, PMID: 25024433

Cesari S. 2018. Multiple strategies for pathogen perception by plant immune receptors. New Phytologist 219 17-24. DOI: https://doi.org/10.1111/nph.14877, PMID: 29131341

Chae E, Bomblies K, Kim ST, Karelina D, Zaidem M, Ossowski S, Martín-Pizarro C, Laitinen RA, Rowan BA, Tenenboim H, Lechner S, Demar M, Habring-Müller A, Lanz C, Rätsch G, Weigel D. 2014. Species-wide genetic incompatibility analysis identifies immune genes as hot spots of deleterious epistasis. Cell 159:1341-1351. DOI: https://doi.org/10.1016/j.cell.2014.10.049, PMID: 25467443

Chae E, Tran DT, Weigel D. 2016. Cooperation and conflict in the plant immune system. PLOS Pathogens 12: e1005452. DOI: https://doi.org/10.1371/journal.ppat.1005452, PMID: 26986469

Chen VB, Arendall WB, Headd JJ, Keedy DA, Immormino RM, Kapral GJ, Murray LW, Richardson JS, Richardson DC. 2010. MolProbity: all-atom structure validation for macromolecular crystallography . Acta Crystallographica Section D Biological Crystallography 66:12-21. DOI: https://doi.org/10.1107/ S0907444909042073

Chen J, Huang Q, Gao D, Wang J, Lang Y, Liu T, Li B, Bai Z, Luis G, Liang C, Chen C, Zhang W, Sun S, Liao Y, Zhang X, Yang L, Song C, Wang M, Shi J, Liu G, et al. 2013. Whole-genome sequencing of Oryza brachyantha reveals mechanisms underlying Oryza genome evolution. Nature Communications 4:1595. DOI: https://doi.org/ 10.1038/ncomms2596, PMID: 23481403

Cohen O, Pupko T. 2011. Inference of gain and loss events from phyletic patterns using stochastic mapping and maximum parsimony-a simulation study. Genome Biology and Evolution 3:1265-1275. DOI: https://doi.org/10. 1093/gbe/evr101, PMID: 21971516

Costanzo S, Jia Y. 2010. Sequence variation at the rice blast resistance gene Pi-km locus: implications for the development of allele specific markers. Plant Science 178:523-530. DOI: https://doi.org/10.1016/j.plantsci. 2010.02.014

Couch BC, Fudal I, Lebrun MH, Tharreau D, Valent B, van Kim P, Nottéghem JL, Kohn LM. 2005. Origins of hostspecific populations of the blast pathogen Magnaporthe oryzae in crop domestication with subsequent expansion of pandemic clones on rice and weeds of rice. Genetics 170:613-630. DOI: https://doi.org/10.1534/ genetics.105.041780, PMID: 15802503

de Abreu-Neto JB, Turchetto-Zolet AC, de Oliveira LF, Zanettini MH, Margis-Pinheiro M. 2013. Heavy metalassociated isoprenylated plant protein (HIPP): characterization of a family of proteins exclusive to plants. FEBS Journal 280:1604-1616. DOI: https://doi.org/10.1111/febs.12159, PMID: 23368984

de Guillen K, Ortiz-Vallejo D, Gracy J, Fournier E, Kroj T, Padilla A. 2015. Structure analysis uncovers a highly diverse but structurally conserved effector family in phytopathogenic fungi. PLOS Pathogens 11:e1005228. DOI: https://doi.org/10.1371/journal.ppat.1005228, PMID: 26506000 
De la Concepcion JC, Franceschetti M, Maqbool A, Saitoh H, Terauchi R, Kamoun S, Banfield MJ. 2018. Publisher correction: polymorphic residues in rice NLRs expand binding and response to effectors of the blast pathogen. Nature Plants 4:734. DOI: https://doi.org/10.1038/s41477-018-0248-0, PMID: 30127412

De la Concepcion JC, Franceschetti M, MacLean D, Terauchi R, Kamoun S, Banfield MJ. 2019. Protein engineering expands the effector recognition profile of a rice NLR immune receptor. eLife 8:e47713. DOI: https://doi.org/10.7554/eLife.47713, PMID: 31535976

De la Concepcion JC, Maidment JHR, Longya A, Xiao G, Franceschetti M, Banfield MJ. 2021. The allelic rice immune receptor pikh confers extended resistance to strains of the blast fungus through a single polymorphism in the effector binding interface. PLOS Pathogens 17:e1009368. DOI: https://doi.org/10.1371/journal.ppat. 1009368, PMID: 33647072

Dean AM, Thornton JW. 2007. Mechanistic approaches to the study of evolution: the functional synthesis. Nature Reviews Genetics 8:675-688. DOI: https://doi.org/10.1038/nrg2160, PMID: 17703238

Delaux PM, Hetherington AJ, Coudert Y, Delwiche C, Dunand C, Gould S, Kenrick P, Li FW, Philippe H, Rensing SA, Rich M, Strullu-Derrien C, de Vries J. 2019. Reconstructing trait evolution in plant evo-devo studies. Current Biology 29:R1110-R1118. DOI: https://doi.org/10.1016/j.cub.2019.09.044, PMID: 31689391

Deng J, Fang L, Zhu X, Zhou B, Zhang T. 2019. A CC-NBS-LRR gene induces hybrid lethality in cotton. Journal of Experimental Botany 70:5145-5156. DOI: https://doi.org/10.1093/jxb/erz312, PMID: 31270546

Dodds PN, Rathjen JP. 2010. Plant immunity: towards an integrated view of plant-pathogen interactions. Nature Reviews Genetics 11:539-548. DOI: https://doi.org/10.1038/nrg2812, PMID: 20585331

Dong S, Stam R, Cano LM, Song J, Sklenar J, Yoshida K, Bozkurt TO, Oliva R, Liu Z, Tian M, Win J, Banfield MJ, Jones AM, van der Hoorn RA, Kamoun S. 2014. Effector specialization in a lineage of the irish potato famine pathogen. Science 343:552-555. DOI: https://doi.org/10.1126/science.1246300, PMID: 24482481

Eddy SR. 1998. Profile hidden markov models. Bioinformatics 14:755-763. DOI: https://doi.org/10.1093/ bioinformatics/14.9.755, PMID: 9918945

Edgar RC. 2004. MUSCLE: multiple sequence alignment with high accuracy and high throughput. Nucleic Acids Research 32:1792-1797. DOI: https://doi.org/10.1093/nar/gkh340

Emsley P, Lohkamp B, Scott WG, Cowtan K. 2010. Features and development of coot. Acta Crystallogr Sect D Biol Crystallogr 66:486-501. DOI: https://doi.org/10.1107/S0907444910007493

Evans PR, Murshudov GN. 2013. How good are my data and what is the resolution? Acta Crystallographica Section D Biological Crystallography 69:1204-1214. DOI: https://doi.org/10.1107/S0907444913000061

Feehan JM, Castel B, Bentham AR, Jones JD. 2020. Plant NLRs get by with a little help from their friends. Current Opinion in Plant Biology 56:99-108. DOl: https://doi.org/10.1016/j.pbi.2020.04.006, PMID: 32554226

Felsenstein J. 1985. Confidence limits on phylogenies: an approach using the bootstrap. Evolution 39:783.

Fujisaki K, Abe Y, Kanzaki E, Ito K, Utsushi H, Saitoh H, Białas A, Banfield MJ, Kamoun S, Terauchi R. 2017. An unconventional NOI/RIN4 domain of a rice NLR protein binds host EXO70 protein to confer fungal immunity. bioRxiv. DOI: https://doi.org/10.1101/239400

Fukuoka S, Saka N, Koga H, Ono K, Shimizu T, Ebana K, Hayashi N, Takahashi A, Hirochika H, Okuno K, Yano M. 2009. Loss of function of a proline-containing protein confers durable disease resistance in rice. Science $\mathbf{3 2 5}$ 998-1001. DOI: https://doi.org/10.1126/science.1175550, PMID: 19696351

Gao Y, Wang W, Zhang T, Gong Z, Zhao H, Han GZ. 2018. Out of Water: the origin and early diversification of plant R-Genes. Plant Physiology 177:82-89. DOI: https://doi.org/10.1104/pp.18.00185, PMID: 29563207

Ginestet C. 2011. ggplot2: elegant graphics for data analysis. Journal of the Royal Statistical Society: Series A 174:245-246. DOI: https://doi.org/10.1111/j.1467-985X.2010.00676_9.x

González-Fuente M, Carrère S, Monachello D, Marsella BG, Cazalé AC, Zischek C, Mitra RM, Rezé N, Cottret L, Mukhtar MS, Lurin C, Noël LD, Peeters N. 2020. EffectorK, a comprehensive resource to mine for Ralstonia, xanthomonas, and other published effector interactors in the Arabidopsis proteome. Molecular Plant Pathology 21:1257-1270. DOI: https://doi.org/10.1111/mpp.12965, PMID: 33245626

Griebel T, Maekawa T, Parker JE. 2014. NOD-like receptor cooperativity in effector-triggered immunity. Trends in Immunology 35:562-570. DOI: https://doi.org/10.1016/j.it.2014.09.005, PMID: 25308923

Guo L, Cesari S, de Guillen K, Chalvon V, Mammri L, Ma M, Meusnier I, Bonnot F, Padilla A, Peng YL, Liu J, Kroj T. 2018. Specific recognition of two MAX effectors by integrated HMA domains in plant immune receptors involves distinct binding surfaces. PNAS 115:11637-11642. DOI: https://doi.org/10.1073/pnas.1810705115, PMID: 30355769

Harms MJ, Thornton JW. 2013. Evolutionary biochemistry: revealing the historical and physical causes of protein properties. Nature Reviews Genetics 14:559-571. DOI: https://doi.org/10.1038/nrg3540, PMID: 23864121

Harris CJ, Slootweg EJ, Goverse A, Baulcombe DC. 2013. Stepwise artificial evolution of a plant disease resistance gene. PNAS 110:21189-21194. DOl: https://doi.org/10.1073/pnas.1311134110, PMID: 24324167

Heidrich K, Tsuda K, Blanvillain-Baufumé S, Wirthmueller L, Bautor J, Parker JE. 2013. Arabidopsis TNL-WRKY domain receptor RRS1 contributes to temperature-conditioned RPS4 auto-immunity. Frontiers in Plant Science 4:403. DOI: https://doi.org/10.3389/fpls.2013.00403, PMID: 24146667

Hodkinson TR. 2018. Evolution and Taxonomy of the Grasses (Poaceae): A Model Family for the Study of Species-Rich Groups Annual Plant Reviews Online. Wiley . DOI: https://doi.org/10.1002/9781119312994. apr0622

Jacob F, Vernaldi S, Maekawa T. 2013. Evolution and conservation of plant NLR functions. Frontiers in Immunology 4:297. DOI: https://doi.org/10.3389/fimmu.2013.00297, PMID: 24093022 
Jacquemin J, Chaparro C, Laudie M, Berger A, Gavory F, Goicoechea JL, Wing RA, Cooke R. 2011. Long-Range and targeted ectopic recombination between the two homeologous chromosomes 11 and 12 in Oryza species. Molecular Biology and Evolution 28:3139-3150. DOl: https://doi.org/10.1093/molbev/msr144

Jia Y, Lee FN, McClung A. 2009. Determination of resistance spectra of the Pi-ta and Pi-k genes to U.S. races of Magnaporthe oryzae causing rice blast in a recombinant inbred line population. Plant Disease 93:639-644. DOI: https://doi.org/10.1094/PDIS-93-6-0639, PMID: 30764397

Jones DT, Taylor WR, Thornton JM. 1992. The rapid generation of mutation data matrices from protein sequences. Bioinformatics 8:275-282. DOI: https://doi.org/10.1093/bioinformatics/8.3.275

Jones JD, Vance RE, Dangl JL. 2016. Intracellular innate immune surveillance devices in plants and animals. Science 354:aaf6395. DOI: https://doi.org/10.1126/science.aaf6395, PMID: 27934708

Kanzaki H, Yoshida K, Saitoh H, Fujisaki K, Hirabuchi A, Alaux L, Fournier E, Tharreau D, Terauchi R. 2012. Arms race co-evolution of Magnaporthe oryzae AVR-Pik and rice pik genes driven by their physical interactions. The Plant Journal : For Cell and Molecular Biology 72:894-907. DOI: https://doi.org/10.1111/j.1365-313X.2012. 05110.x, PMID: 22805093

Karasov TL, Kniskern JM, Gao L, DeYoung BJ, Ding J, Dubiella U, Lastra RO, Nallu S, Roux F, Innes RW, Barrett LG, Hudson RR, Bergelson J. 2014. The long-term maintenance of a resistance polymorphism through diffuse interactions. Nature 512:436-440. DOI: https://doi.org/10.1038/nature13439, PMID: 25043057

Kourelis J, Kamoun S. 2020. RefPlantNLR: a comprehensive collection of experimentally validated plant NLRs. bioRxiv. DOI: https://doi.org/10.1101/2020.07.08.193961

Kourelis J, van der Hoorn RAL. 2018. Defended to the nines: 25 years of resistance gene cloning identifies nine mechanisms for R protein function. The Plant Cell 30::285-299. DOI: https://doi.org/10.1105/tpc.17.00579

Kroj T, Chanclud E, Michel-Romiti C, Grand X, Morel JB. 2016. Integration of decoy domains derived from protein targets of pathogen effectors into plant immune receptors is widespread. New Phytologist 210:618626. DOI: https://doi.org/10.1111/nph.13869, PMID: 26848538

Kuang H, Woo S-S, Meyers BC, Nevo E, Michelmore RW. 2004. Multiple genetic processes result in heterogeneous rates of evolution within the major cluster disease resistance genes in lettuce[W]. The Plant Cell 16:2870-2894. DOI: https://doi.org/10.1105/tpc.104.025502

Kumar S, Stecher G, Suleski M, Hedges SB. 2017. TimeTree: a resource for timelines, timetrees, and divergence times. Molecular Biology and Evolution 34:1812-1819. DOI: https://doi.org/10.1093/molbev/msx116

Kumar S, Stecher G, Li M, Knyaz C, Tamura K. 2018. MEGA X: molecular evolutionary genetics analysis across computing platforms. Molecular Biology and Evolution 35:1547-1549. DOI: https://doi.org/10.1093/molbev/ msy096, PMID: 29722887

Kurata N, Yamazaki Y. 2006. Oryzabase an integrated biological and genome information database for rice. Plant Physiology 140:12-17. DOI: https://doi.org/10.1104/pp.105.063008, PMID: 16403737

Langner T, Harant A, Gomez-Luciano LB, Shrestha RK, Malmgren A, Latorre SM, Burbano HA, Win J, Kamoun S. 2021. Genomic rearrangements generate hypervariable mini-chromosomes in host-specific isolates of the blast fungus. PLOS Genetics 17:e1009386. DOI: https://doi.org/10.1371/journal.pgen.1009386, PMID: 33591993

Latorre SM, Reyes-Avila CS, Malmgren A, Win J, Kamoun S, Burbano HA. 2020. Differential loss of effector genes in three recently expanded pandemic clonal lineages of the rice blast fungus. BMC Biology 18:88. DOI: https://doi.org/10.1186/s12915-020-00818-z, PMID: 32677941

Lee RRQ, Chae E. 2020. Variation patterns of NLR clusters in Arabidopsis thaliana genomes. Plant Communications 1:100089. DOI: https://doi.org/10.1016/j.xplc.2020.100089, PMID: 33367252

Letunic I, Bork P. 2007. Interactive tree of life (iTOL): an online tool for phylogenetic tree display and annotation. Bioinformatics 23:127-128. DOI: https://doi.org/10.1093/bioinformatics/btl529, PMID: 17050570

Li J, Wang Q, Li C, Bi Y, Fu X, Wang R. 2019. Novel haplotypes and networks of AVR-Pik alleles in Magnaporthe oryzae. BMC Plant Biology 19:204. DOI: https://doi.org/10.1186/s12870-019-1817-8, PMID: 31096914

Li J, Zhang M, Sun J, Mao X, Wang J, Liu H, Zheng H, Li X, Zhao H, Zou D. 2020a. Heavy metal Stress-Associated proteins in rice and Arabidopsis: Genome-Wide Identification, Phylogenetics, Duplication, and Expression Profiles Analysis. Frontiers in Genetics 11:477. DOI: https://doi.org/10.3389/fgene.2020.00477, PMID: 3245780 8

Li L, Habring A, Wang K, Weigel D. 2020b. Atypical resistance protein RPW8/HR triggers oligomerization of the NLR immune receptor RPP7 and autoimmunity. Cell Host \& Microbe 27:405-417. DOI: https://doi.org/10.1016/ j.chom.2020.01.012

Longya A, Chaipanya C, Franceschetti M, Maidment JHR, Banfield MJ, Jantasuriyarat C. 2019. Gene duplication and mutation in the emergence of a novel aggressive allele of the AVR-Pik Effector in the Rice Blast Fungus. Molecular Plant-Microbe Interactions 32:740-749. DOI: https://doi.org/10.1094/MPMI-09-18-0245-R, PMID: 30601714

Lukasik-Shreepaathy E, Slootweg E, Richter H, Goverse A, Cornelissen BJ, Takken FL. 2012. Dual regulatory roles of the extended $\mathrm{N}$ terminus for activation of the tomato Ml-1.2 resistance protein. Molecular PlantMicrobe Interactions 25:1045-1057. DOI: https://doi.org/10.1094/MPMI-11-11-0302, PMID: 22512381

MacLean D. 2019. Besthr. https://besthr.co.uk/

Maekawa T, Kracher B, Saur IML, Yoshikawa-Maekawa M, Kellner R, Pankin A, von Korff M, Schulze-Lefert P. 2019. Subfamily-Specific specialization of RGH1/MLA immune receptors in wild barley. Molecular Plant-Microbe Interactions 32:107-119. DOI: https://doi.org/10.1094/MPMI-07-18-0186-FI, PMID: 30295580

Maidment JHR, Franceschetti M, Maqbool A, Saitoh H. 2020. Multiple variants of the blast fungus effector AVRPik bind the HMA domain of the rice protein OsHIPP19 with high affinity. bioRxiv. DOI: https://doi.org/10.

$1101 / 2020.12 .01 .403451$ 
Maqbool A, Saitoh H, Franceschetti M, Stevenson CEM, Uemura A, Kanzaki H, Kamoun S, Terauchi R, Banfield MJ. 2015. Structural basis of pathogen recognition by an integrated HMA domain in a plant NLR immune receptor. eLife 4:e08709. DOI: https://doi.org/10.7554/eLife.08709

McNicholas S, Potterton E, Wilson KS, Noble ME. 2011. Presenting your structures: the CCP4mg moleculargraphics software. Acta Crystallogr Sect D Biol Crystallogr 40:386-394. DOI: https://doi.org/10.1107/ S0907444911007281

Mizuno H, Katagiri S, Kanamori H, Mukai Y, Sasaki T, Matsumoto T, Wu J. 2020. Evolutionary dynamics and impacts of chromosome regions carrying R-gene clusters in rice. Scientific Reports 10:1-2. DOI: https://doi.org/ 10.1038/s41598-020-57729-w

Moscou M. 2019. OKphylogeny. Github. 36ad9a4 . https://github.com/matthewmoscou/QKphylogeny

Murshudov GN, Skubák P, Lebedev AA, Pannu NS, Steiner RA, Nicholls RA, Winn MD, Long F, Vagin AA. 2011. REFMAC 5 for the refinement of macromolecular crystal structures. Acta Crystallographica Section D Biological Crystallography 67:355-367. DOI: https://doi.org/10.1107/S0907444911001314

Myszka DG. 1999. Improving biosensor analysis. Journal of Molecular Recognition 12:279-284. DOI: https://doi. org/10.1002/(SICI)1099-1352(199909/10)12:5<279::AID-JMR473>3.0.CO;2-3, PMID: 10556875

Nei M, Gojobori T. 1986. Simple methods for estimating the numbers of synonymous and nonsynonymous nucleotide substitutions. Molecular Biology and Evolution 3:418-426. DOl: https://doi.org/10.1093/ oxfordjournals.molbev.a040410, PMID: 3444411

Oikawa K, Fujisaki K, Shimizu M, Takeda T, Saitoh H, Hirabuchi A, Hiraka Y, Białas A, Langner T, Kellner R, Bozkurt T, Cesari S, Kroj T, Maidment J, Banfield M, Kamoun S, Terauchi R. 2020. The blast pathogen effector AVR-Pik binds and stabilizes rice heavy metal-associated (HMA) proteins to co-opt their function in immunity. bioRxiv. DOI: https://doi.org/10.1101/2020.12.01.406389

Okuyama Y, Kanzaki H, Abe A, Yoshida K, Tamiru M, Saitoh H, Fujibe T, Matsumura H, Shenton M, Galam DC, Undan J, Ito A, Sone T, Terauchi R. 2011. A multifaceted genomics approach allows the isolation of the rice Pia-blast resistance gene consisting of two adjacent NBS-LRR protein genes. The Plant Journal 66:467-479. DOI: https://doi.org/10.1111/j.1365-313X.2011.04502.x

Petit-Houdenot Y, Langner T, Harant A, Win J, Kamoun S. 2020. A clone resource of Magnaporthe oryzae Effectors That Share Sequence and Structural Similarities Across Host-Specific Lineages. Molecular PlantMicrobe Interactions 33:1032-1035. DOI: https://doi.org/10.1094/MPMI-03-20-0052-A, PMID: 32460610

Potterton L, Agirre J, Ballard C, Cowtan K, Dodson E, Evans PR, Jenkins HT, Keegan R, Krissinel E, Stevenson K, Lebedev A, McNicholas SJ, Nicholls RA, Noble M, Pannu NS, Roth C, Sheldrick G, Skubak P, Turkenburg J, Uski V, et al. 2018. CCP 4 i 2: the new graphical user interface to the CCP 4 program suite. Acta Crystallogr Sect D Struct Biol 74:68-84. DOI: https://doi.org/10.1107/S2059798317016035

Prigozhin DM, Krasileva KV. 2020. Intraspecies diversity reveals a subset of highly variable plant immune receptors and predicts their binding sites. bioRxiv. DOl: https://doi.org/10.1101/2020.07.10.190785

Pupko T, Pe'er I, Shamir R, Graur D. 2000. A fast algorithm for joint reconstruction of ancestral amino acid sequences. Molecular Biology and Evolution 17:890-896. DOl: https://doi.org/10.1093/oxfordjournals.molbev. a026369, PMID: 10833195

Qi D, DeYoung BJ, Innes RW. 2012. Structure-function analysis of the coiled-coil and leucine-rich repeat domains of the RPS5 disease resistance protein. Plant Physiology 158:1819-1832. DOI: https://doi.org/10.1104/pp.112. 194035, PMID: 22331412

Radakovic ZS, Anjam MS, Escobar E, Chopra D, Cabrera J, Silva AC, Escobar C, Sobczak M, Grundler FMW, Siddique S. 2018. Arabidopsis HIPP27 is a host susceptibility gene for the beet cyst nematode Heterodera schachtii. Molecular Plant Pathology 19:1917-1928. DOI: https://doi.org/10.1111/mpp.12668

Rairdan GJ, Moffett P. 2006. Distinct domains in the ARC region of the potato resistance protein rx mediate LRR binding and inhibition of activation. The Plant Cell 18:2082-2093. DOI: https://doi.org/10.1105/tpc.106. 042747, PMID: 16844906

Randall RN, Radford CE, Roof KA, Natarajan DK, Gaucher EA. 2016. An experimental phylogeny to benchmark ancestral sequence reconstruction. Nature Communications 7:1-6. DOI: https://doi.org/10.1038/ncomms12847

Read AC, Moscou MJ, Zimin AV, Pertea G, Meyer RS, Purugganan MD, Leach JE, Triplett LR, Salzberg SL, Bogdanove AJ. 2020. Genome assembly and characterization of a complex zfBED-NLR gene-containing disease resistance locus in Carolina gold select rice with nanopore sequencing. PLOS Genetics 16:e1008571. DOI: https://doi.org/10.1371/journal.pgen.1008571, PMID: 31986137

Saitou N, Nei M. 1987. The neighbor-joining method: a new method for reconstructing phylogenetic trees. Molecular Biology and Evolution 4:406-425. DOI: https://doi.org/10.1093/oxfordjournals.molbev.a040454, PMID: 3447015

Sarris PF, Cevik V, Dagdas G, Jones JD, Krasileva KV. 2016. Comparative analysis of plant immune receptor architectures uncovers host proteins likely targeted by pathogens. BMC Biology 14:8. DOI: https://doi.org/10. 1186/s12915-016-0228-7, PMID: 26891798

Saucet SB, Ma Y, Sarris PF, Furzer OJ, Sohn KH, Jones JDG. 2015. Two linked pairs of Arabidopsis TNL resistance genes independently confer recognition of bacterial effector AvrRps4. Nature Communications 6:112. DOI: https://doi.org/10.1038/ncomms7338

Seeholzer S, Tsuchimatsu T, Jordan T, Bieri S, Pajonk S, Yang W, Jahoor A, Shimizu KK, Keller B, Schulze-Lefert P. 2010. Diversity at the mla powdery mildew resistance locus from cultivated barley reveals sites of positive selection. Molecular Plant-Microbe Interactions 23:497-509. DOI: https://doi.org/10.1094/MPMI-23-4-0497, PMID: 20192836 
Segretin ME, Pais M, Franceschetti M, Chaparro-Garcia A, Bos JI, Banfield MJ, Kamoun S. 2014. Single amino acid mutations in the potato immune receptor R3a expand response to Phytophthora effectors. Molecular Plant-Microbe Interactions 27:624-637. DOI: https://doi.org/10.1094/MPMI-02-14-0040-R, PMID: 24678835

Shao ZQ, Xue JY, Wu P, Zhang YM, Wu Y, Hang YY, Wang B, Chen JQ. 2016. Large-Scale analyses of angiosperm Nucleotide-Binding Site-Leucine-Rich repeat genes reveal three anciently diverged classes with distinct evolutionary patterns. Plant Physiology 170:2095-2109. DOI: https://doi.org/10.1104/pp.15.01487, PMID: 26839128

Sinapidou E, Williams K, Nott L, Bahkt S, Tör M, Crute I, Bittner-Eddy P, Beynon J. 2004. Two TIR:nb:Irr genes are required to specify resistance to Peronospora parasitica isolate Cala2 in Arabidopsis. The Plant Journal : For Cell and Molecular Biology 38:898-909. DOI: https://doi.org/10.1111/j.1365-313X.2004.02099.x, PMID: 15165183

Stamatakis A. 2014. RAxML version 8: a tool for phylogenetic analysis and post-analysis of large phylogenies. Bioinformatics 30:1312-1313. DOI: https://doi.org/10.1093/bioinformatics/btu033, PMID: 24451623

Stein JC, Yu Y, Copetti D, Zwickl DJ, Zhang L, Zhang C, Chougule K, Gao D, Iwata A, Goicoechea JL, Wei S, Wang J, Liao Y, Wang M, Jacquemin J, Becker C, Kudrna D, Zhang J, Londono CEM, Song X, et al. 2018. Genomes of 13 domesticated and wild rice relatives highlight genetic conservation, turnover and innovation across the genus Oryza. Nature Genetics 50:285-296. DOl: https://doi.org/10.1038/s41588-018-0040-0, PMID: 29358651

Steuernagel B, Jupe F, Witek K, Jones JD, Wulff BB. 2015. NLR-parser: rapid annotation of plant NLR complements. Bioinformatics 31:1665-1667. DOI: https://doi.org/10.1093/bioinformatics/btv005, PMID: 255 86514

Tanaka S, Schweizer G, Rössel N, Fukada F, Thines M, Kahmann R. 2019. Neofunctionalization of the secreted Tin2 effector in the fungal pathogen Ustilago maydis. Nature Microbiology 4:251-257. DOI: https://doi.org/10. 1038/s41564-018-0304-6, PMID: 30510169

Tavaré S. 1986. Some probabilistic and statistical problems in the analysis of DNA sequences. Biology 17:86.

Thornton JW. 2004. Resurrecting ancient genes: experimental analysis of extinct molecules. Nature Reviews Genetics 5:366-375. DOI: https://doi.org/10.1038/nrg1324, PMID: 15143319

Tran DTN, Chung EH, Habring-Müller A, Demar M, Schwab R, Dangl JL, Weigel D, Chae E. 2017. Activation of a plant NLR complex through heteromeric association with an autoimmune risk variant of another NLR. Current Biology 27:1148-1160. DOI: https://doi.org/10.1016/j.cub.2017.03.018, PMID: 28416116

Van de Weyer AL, Monteiro F, Furzer OJ, Nishimura MT, Cevik V, Witek K, Jones JDG, Dangl JL, Weigel D, Bemm F. 2019. A Species-Wide inventory of NLR genes and alleles in Arabidopsis thaliana. Cell 178:12601272. DOI: https://doi.org/10.1016/j.cell.2019.07.038, PMID: 31442410

Varden FA, Saitoh H, Yoshino K, Franceschetti M, Kamoun S, Terauchi R, Banfield MJ. 2019. Cross-reactivity of a rice NLR immune receptor to distinct effectors from the rice blast pathogen Magnaporthe oryzae provides partial disease resistance. Journal of Biological Chemistry 294:13006-13016. DOl: https://doi.org/10.1074/jbc. RA119.007730, PMID: 31296569

Wang L, Xu X, Lin F, Pan Q. 2009. Characterization of rice blast resistance genes in the pik cluster and fine mapping of the Pik-p locus. Phytopathology 99:900-905. DOI: https://doi.org/10.1094/PHYTO-99-8-0900, PMID: 19594308

Wang GF, Ji J, El-Kasmi F, Dangl JL, Johal G, Balint-Kurti PJ. 2015. Molecular and functional analyses of a maize autoactive NB-LRR protein identify precise structural requirements for activity. PLOS Pathogens 11:e1004674. DOI: https://doi.org/10.1371/journal.ppat.1004674, PMID: 25719542

Waterhouse A, Bertoni M, Bienert S, Studer G, Tauriello G, Gumienny R, Heer FT, de Beer TAP, Rempfer C, Bordoli L, Lepore R, Schwede T. 2018. SWISS-MODEL: homology modelling of protein structures and complexes. Nucleic Acids Research 46:W296-W303. DOI: https://doi.org/10.1093/nar/gky427, PMID: 297 88355

Weber E, Engler C, Gruetzner R, Werner S, Marillonnet S. 2011. A modular cloning system for standardized assembly of multigene constructs. PLOS ONE 6:e16765. DOI: https://doi.org/10.1371/journal.pone.0016765, PMID: 21364738

Win J, Kamoun S, Jones AME. 2011. Purification of Effector-Target Protein Complexes via Transient Expression in Nicotiana Benthamiana, Plant Immunity, Methods in Molecular Biology. Totowa, NJ: Humana Press. DOI: https://doi.org/10.1007/978-1-61737-998-7_15

Win J, Kamoun S. 2003. pCB301-p19: a binary plasmid vector to enhance transient expression of transgenes by agroinfiltration. The Plant Journal : For Cell and Molecular Biology 33:949-956.

Winter G. 2010. xia2 : an expert system for macromolecular crystallography data reduction . Journal of Applied Crystallography 43:186-190. DOI: https://doi.org/10.1107/S0021889809045701

Wu CH, Krasileva KV, Banfield MJ, Terauchi R, Kamoun S. 2015. The "sensor domains" of plant NLR proteins: more than decoys? Frontiers in Plant Science 6:134. DOI: https://doi.org/10.3389/fpls.2015.00134, PMID: 257 98142

Wu CH, Derevnina L, Kamoun S. 2018. Receptor networks underpin plant immunity. Science 360:1300-1301. DOI: https://doi.org/10.1126/science.aat2623, PMID: 29930125

Yamamoto E, Takashi T, Morinaka Y, Lin S, Wu J, Matsumoto T, Kitano H, Matsuoka M, Ashikari M. 2010. Gain of deleterious function causes an autoimmune response and Bateson-Dobzhansky-Muller incompatibility in rice. Molecular Genetics and Genomics 283:305-315. DOI: https://doi.org/10.1007/s00438-010-0514-y, PMID: 20140455 
Yang Z. 1997. PAML: a program package for phylogenetic analysis by maximum likelihood. Bioinformatics 13 : 555-556. DOI: https://doi.org/10.1093/bioinformatics/13.5.555

Yang Z. 2000. Maximum likelihood estimation on large phylogenies and analysis of adaptive evolution in human influenza virus A. Journal of Molecular Evolution 51:423-432. DOl: https://doi.org/10.1007/s002390010105, PMID: 11080365

Yang Z, Swanson WJ, Vacquier VD. 2000. Maximum-likelihood analysis of molecular adaptation in abalone sperm lysin reveals variable selective pressures among lineages and sites. Molecular Biology and Evolution 17:14461455. DOI: https://doi.org/10.1093/oxfordjournals.molbev.a026245, PMID: 11018152

Yang Z, Wong WS, Nielsen R. 2005. Bayes empirical bayes inference of amino acid sites under positive selection. Molecular Biology and Evolution 22:1107-1118. DOI: https://doi.org/10.1093/molbev/msi097, PMID: 15689528

Yang Z, Nielsen R. 1998. Synonymous and nonsynonymous rate variation in nuclear genes of mammals. Journal of Molecular Evolution 46:409-418. DOI: https://doi.org/10.1007/PL00006320, PMID: 9541535

Yang Z, Nielsen R. 2000. Estimating synonymous and nonsynonymous substitution rates under realistic evolutionary models. Molecular Biology and Evolution 17:32-43. DOI: https://doi.org/10.1093/oxfordjournals. molbev.a026236, PMID: 10666704

Yoshida K, Saunders DG, Mitsuoka C, Natsume S, Kosugi S, Saitoh H, Inoue Y, Chuma I, Tosa Y, Cano LM, Kamoun S, Terauchi R. 2016. Host specialization of the blast fungus Magnaporthe oryzae is associated with dynamic gain and loss of genes linked to transposable elements. BMC Genomics 17:370. DOI: https://doi.org/ 10.1186/s12864-016-2690-6, PMID: 27194050

Yuan B, Zhai C, Wang W, Zeng X, Xu X, Hu H, Lin F, Wang L, Pan Q. 2011. The Pik-p resistance to Magnaporthe oryzae in rice is mediated by a pair of closely linked CC-NBS-LRR genes. Theoretical and Applied Genetics 122: 1017-1028. DOI: https://doi.org/10.1007/s00122-010-1506-3, PMID: 21153625

Zdrzałek R, Kamoun S, Terauchi R, Saitoh H, Banfield MJ. 2020. The rice NLR pair Pikp-1/Pikp-2 initiates cell death through receptor cooperation rather than negative regulation. PLOS ONE 15:e0238616. DOI: https:// doi.org/10.1371/journal.pone.0238616, PMID: 32931489

Zess EK, Białas A, Kamoun S. 2019. Old fungus, new trick. Nature Microbiology 4:210-211. DOI: https://doi.org/ 10.1038/s41564-018-0351-z, PMID: 30675032

Zhai C, Lin F, Dong Z, He X, Yuan B, Zeng X, Wang L, Pan Q. 2011. The isolation and characterization of pik, a rice blast resistance gene which emerged after rice domestication. New Phytologist 189:321-334. DOI: https:// doi.org/10.1111/j.1469-8137.2010.03462.x, PMID: 21118257

Zhai C, Zhang Y, Yao N, Lin F, Liu Z, Dong Z, Wang L, Pan Q. 2014. Function and interaction of the coupled genes responsible for Pik-h encoded rice blast resistance. PLOS ONE 9:e98067. DOl: https://doi.org/10.1371/ journal.pone.0098067, PMID: 24896089

Zschiesche W, Barth O, Daniel K, Böhme S, Rausche J, Humbeck K. 2015. The zinc-binding nuclear protein HIPP3 acts as an upstream regulator of the salicylate-dependent plant immunity pathway and of flowering time in Arabidopsis thaliana. New Phytologist 207:1084-1096. DOI: https://doi.org/10.1111/nph.13419, PMID: 25 913773 